\title{
A lower X-gate in TASK channels traps inhibitors within the vestibule
}

\section{Authors}

Karin E. J. Rödström,"\#, Aytuğ K. Kiper,"\#, Wei Zhang ${ }^{1,5}$, Susanne Rinné2 , Ashley C. W. Pike, Matthias Goldstein ${ }^{2}$, Linus J. Conrad ${ }^{3,6}$, Martina Delbeck ${ }^{4}$, Michael Hahn ${ }^{4}$, Heinrich Meier ${ }^{4}$, Magdalena Platzk ${ }^{4}$, Andrew Quigley ${ }^{1,7}$, David Speedman ${ }^{1}$, Leela Shrestha ${ }^{1}$, Shubhashish M.M. Mukhopadhyay $^{1}$, Nicola A. Burgess-Brown ${ }^{1}$, Stephen J. Tucker ${ }^{3}$, Thomas Mueller ${ }^{4}$, Niels Decher $^{2, \S, *}$, Elisabeth P. Carpenter ${ }^{1, \S, *}$.

${ }^{1}$ Structural Genomics Consortium, University of Oxford, Oxford, OX3 7DQ, UK.

${ }^{2}$ Institute for Physiology and Pathophysiology, Vegetative Physiology, University of Marburg, Marburg, Germany.

${ }^{3}$ Department of Physics, University of Oxford, OX1 3QT, UK.

${ }^{4}$ Bayer AG, Research \& Development, Pharmaceuticals, Wuppertal, Germany.

${ }^{5}$ Present address: Public Technology Service Center and CAS Key Laboratory of Pathogenic Microbiology and Immunology, Institute of Microbiology, Chinese Academy of Sciences, Beijing 100101, China.

${ }^{6}$ Present address: Department of Biomedical Science, University of Sheffield, Sheffield, S10 2TN, UK.

${ }^{7}$ Present address: Membrane Protein Laboratory, Research Complex at Harwell, Harwell, UK.

\# These authors contributed equally to this work.

$\S$ These authors jointly supervised this work.

*e-mail: decher@staff.uni-marburg.de, liz.carpenter@sgc.ox.ac.uk 
TASK channels are unusual members of the two-pore domain potassium $\left(\mathrm{K}_{2 \mathrm{P}}\right)$ channel family, found in neurons ${ }^{1}$, cardiomyocytes $^{2-4}$ and vascular smooth muscle cells ${ }^{5}$ where they are involved in regulation of heart rate $^{6}$, pulmonary artery tone ${ }^{5,7}$, sleep/wake cycles ${ }^{8}$ and responses to volatile anaesthetics ${ }^{8-11}$. K2P channels regulate the resting membrane potential, providing background $\mathrm{K}^{+}$currents controlled by numerous physiological stimuli $^{12-15}$. Unlike other $K_{2 P}$ channels, TASK channels have the capacity to bind inhibitors with high affinity, exceptional selectivity and very slow compound washout rates. These characteristics make TASK channels some of the most easily druggable potassium channels, and indeed TASK-1 inhibitors are currently in clinical trials for obstructive sleep apnea (OSA) and atrial fibrillation (Afib) ${ }^{16}$ (the DOCTOS and SANDMAN trials). Generally, potassium channels have an intramembrane vestibule with a selectivity filter above and a gate with four parallel helices below. However, $K_{2 P}$ channels studied to date all lack a lower gate. Here we present the structure of TASK-1, revealing a lower gate created by interaction of the two crossed C-terminal M4 transmembrane helices at the vestibule entrance, which we designate as an "X-gate". This structure is formed by six residues $\left(\mathrm{V}^{243} \mathrm{LRFMT}^{248}\right)$ that are essential for responses to volatile anaesthetics $^{10}$, neuro-transmitters ${ }^{13}$ and G-protein coupled receptors ${ }^{13}$. Interestingly, mutations within the $\mathrm{X}$-gate and surrounding regions drastically affect both open probability and activation by anaesthetics. Structures of TASK-1 with two novel, highaffinity blockers, show both inhibitors bound below the selectivity filter, trapped in the vestibule by the $\mathrm{X}$-gate, thus explaining their exceptionally low wash-out rates. Thus, the presence of the X-gate in TASK channels explains many aspects of their unusual physiological and pharmacological behaviour, which is invaluable for future development and optimization of TASK modulators for treatment of heart, lung and sleep disorders. 
To understand the molecular basis of the unusual properties of TASK channels we solved the structure of human TWIK-related acid-sensitive potassium channel 1 (TASK-1, $\mathrm{K}_{2 \mathrm{P} 3.1)}$ by X-ray crystallography. We used a C-terminally truncated construct (Met1 to Glu259) for the structural studies, which had similar properties to the full-length channel in its responses to the pore-blockers A1899 and ML365, and to inhibition by $\mathrm{H}^{+}$ions (Extended Data Fig. 1ac). We determined the structure using X-ray data to $3.0 \AA$ A resolution (Methods, Extended Data Table 1, Fig. 1, Extended Data Fig. 2a-g). TASK-1 forms a domain-swapped homodimer (Fig. 1a), with a pseudo-tetrameric selectivity filter, four transmembrane helices (M1-M4) and an extracellular cap, similar to structures of other $\mathrm{K}_{2 \mathrm{PS}}{ }^{17-21}$ (Fig. 1b). The selectivity filter resembles that of other $\mathrm{K}_{2 \mathrm{P}}$ channels, with binding sites for four $\mathrm{K}^{+}$ions (Fig. 1c-e, Extended Data Fig. 2b). TASK-1 is inhibited by high extracellular $\mathrm{H}^{+}$concentrations (acidic $\left.\mathrm{pHs}\right)^{12}$ (Extended Data Fig. 1c,d) and His98, near the selectivity filter, is reported to be the extracellular $\mathrm{pH}$ sensor $^{22}$. Crystals were grown at $\mathrm{pH} 8.5$ and the structure reveals that His98 plays an important role in stabilizing the region behind the selectivity filter, including forming a hydrogen bond to a key water molecule behind the filter (Extended Data Fig. 2c,d). Since TASK-1 is active at $\mathrm{pH} 8.5$, the state shown here is likely to represent the active, deprotonated state of His98 and the selectivity filter, similar to that proposed in modelling studies ${ }^{23}$, although the overall state of the channel is closed, as shown below. His98 is positioned below the two hydrogen-bonded sidechains of Gln77 and Gln209, and mutation of these residues indicated that Gln209 influences the sensitivity of TASK-1 to $\mathrm{H}^{+}$ions (Extended Data Fig. 1e,f).

One unusual feature of TASK and THIK channels is the absence of the intermolecular disulphide bond at the apex of the external cap domain (Extended Data Fig. 3a). We found that in TASK-1 the domain-swapped cap structure is preserved without this disulphide bond (Extended Data Fig. 2f-i), as previously predicted ${ }^{24}$. Therefore, this feature does not explain the differences in TASK-1 properties. The relative positions of the M2-M4 helices determine whether stretch-activated $\mathrm{K}_{2 \mathrm{P}}$ channels are in a more active "up" state with raised helices and 
closed fenestrations, or a lower activity "down" state with open fenestrations ${ }^{19,20}$. Overall the TASK-1 structure adopts a "down"-like state, with some adjustment of the M2 position and open fenestrations connecting the vestibule to the lipid bilayer (Fig. 1a-e).

Surprisingly, we found that at the distal end of M4 adopts an unexpected conformation, causing these helices to lie across the entrance of the vestibule, forming a closed gate which we have designated as an "X-gate" (Fig. 1c-h). This conformation is caused by bends in M4 at residues Leu241 and Asn250, on either side of a six residue sequence $\left(V^{243} \mathrm{LRFMT}^{248}\right)$ which forms the X-gate. This leaves a narrow gap between the M4 helices, which would allow spheres of no more than $0.8 \AA$ radius to pass through the gate, in contrast to TREK-2 (PDB:4XDJ), which has an open vestibule allowing spheres of up $7.5 \AA$ radius to pass ( $\mathrm{CHAP}^{25}$ and methods). The X-gate adopts an unusual extended two-turn helical structure, lacking the hydrogen bonding patterns seen in $\alpha$ or $3_{10}$ helices (Extended Data Fig. 2j). The interface is lined by the hydrophobic sidechains of Leu244, Met247 and the methyl group of Thr248 (Fig. 1g,h), which block the ion conduction pathway. Interestingly, this region is essential for TASK-1 responses to volatile anaesthetics ${ }^{13}$, neurotransmitters ${ }^{13}$, $\operatorname{drugs}^{26}$ and G-protein coupled receptors ${ }^{13}$ (potentially through diacylglycerol ${ }^{15}$ or $\mathrm{PIP}_{2}$ interactions ${ }^{14}$ ). Sequence alignments suggest TASK-3 and TASK-5 may also form similar structures (Extended Data Fig. 3b,c). The presence of the X-gate in the TASK subfamily is an exception to the paradigm that $\mathrm{K}_{2 \mathrm{P}}$ channels lack a lower gate ${ }^{27}$. Surprisingly, the structure of the dimeric X-gate is unrelated to the helix bundle crossing' gates with four parallel helices that are typically found in tetrameric $\mathrm{K}^{+}$channels.

To determine whether the X-gate explains the unusual properties of TASK channels, we performed alanine scanning mutagenesis across the distal M4 helix (Thr230-Thr248), and assessed channel activity and surface expression of the mutants. We predicted that disruption of the X-gate would increase the very low TASK-1 basal channel activity ${ }^{3,28}$. Strikingly, mutation of any residue within the X-gate (except Val243), led to increased currents (Fig. 2a,b) without increased surface expression (Extended Data Fig. 4a). The centre of the X-gate is 
formed by Leu244, which is packed between residues on M2, M4 and M4' (Fig. 1h) and the Leu244Ala mutation gave the largest current increase (Fig. 2a,b). Inside out single channel recordings of Leu244Ala confirmed a direct effect on channel gating with a 6-fold increase in channel open probability from 0.7 to $4.0 \%$ (Fig. 2c-e) and increased channel open times (Fig. 2f). Mutation of the equivalent residue in TASK-3 (Leu244Ala) also resulted in increased channel activity (Extended Data Fig. 4b). The X-gate is also important for activation by volatile anaesthetics such as halothane and sevoflurane ${ }^{13}$, and was originally called the "halothane response element". Here we confirm that sevoflurane, a widely used anaesthetic, also activates TASK-1 and that mutations in the X-gate reduce this activation (Fig. 2g, Extended Data Fig. $4 c)$, indicating a role for the $\mathrm{X}$-gate in responses to volatile anaesthetics.

Next we investigated whether residues surrounding the X-gate, were important its formation and stability. The X-gate is preceded by a hinge point in M4 around Leu241-Val242, where the helix axis is bent by $\sim 40^{\circ}$ (Fig. 3a). After the bend the X-gate itself adopts an expanded helical conformation (Extended Data Fig. 2j). The side chain of Leu241 inserts into a hydrophobic pocket, pulling it away from the helix axis and stabilizing the bend in M4. This exposes the Leu241 carbonyl, which forms a hydrogen bond with the Arg245 sidechain (Fig. 3a). Mutation of either Leu241 or Arg245 to Ala increased channel activity by 5-7 fold (Fig. 2a,b), confirming the importance of these interactions. Furthermore, a hydrogen bond between the carbonyl oxygen of Ala237 on M4 and Asn133 on M2 also disrupts the M4 $\alpha$-helix, stabilizing the bend, and mutation of Asn133 increased channel activity (Fig. 3b).

Following the X-gate, a second bend in M4 at residue Asn250 allows its distal end to adopt an $\alpha$-helical structure which forms extensive interactions with the opposite side of the vestibule, a region we named the "latch" (Fig. 3a). This extensive network of salt bridges and hydrogen bonds link M4 to M1 and M2' (Fig. 3a). Mutation of residues across the proximal M1, distal M2' and distal M4 regions gave increased channel activity, consistent with the latch region playing a role in stabilizing the X-gate (Fig. 3b). In particular, Arg7 and Arg131' appear 
to be important for the integrity of the latch and channel gating, since mutations to acidic residues cause a 5 - 10 fold increase in currents (Fig. 3c, Extended Data Fig. 4d), without increased surface expression (Extended data Fig. 4e). It is possible that the opening of the Xgate is coupled to the extracellular $\mathrm{pH}$ sensor. However, mutations in the latch (Arg7Asp and Arg131Asp) had no effect on modulation of TASK-1 channels by extracellular protons, suggesting that the two processes may not be strictly coupled (Extended Data Fig. 4f).

Lipids are important regulators of $\mathrm{K}_{2 \mathrm{P}}$ channels and the structures reveal binding sites for lipids, including the cholesterol analogue cholesteryl hemisuccinate (CHS) (Extended Data Fig. 2e). CHS (added during purification) binds in two places, with one molecule extending along the X-gate, interacting with M1, M4 and M2' (Fig. 3a). Addition of CHS to TASK-1 in inside-out patches did not affect TASK-1 function (Extended Data Fig. 4g,h), whereas depletion of cholesterol by $\beta$-cyclodextrin reduced the channel activity (Extended Data Fig. 4i,j), consistent with a reduction in TASK-1 stability upon cholesterol removal. Phe246 in the X-gate lies next to the CHS molecule (Fig. 3a), and the Phe246Ala mutation produces a 4-fold increase in current (Fig. 2b).

Unlike many other $\mathrm{K}_{2 \mathrm{P}}$ channels that exhibit very poor pharmacology, several high affinity, selective inhibitors exist for TASK-1 and TASK-3. These blockers exhibit exceptionally low wash-out rates after removal of the inhibitor. Together, these characteristics make TASK inhibitors interesting tools to investigate the physiological and pharmacological function of TASK channels. Therefore, we performed an ultra-high throughput screen (uHTS) on TASK-1, which identified two highly potent and selective inhibitors, BAY1000493 and BAY2341237, with EC 50 values of 9.5 and 7.6 nM (Fig. 4a,b,h,i and Extended Data Fig. 5a-d; for additional examples see Extended Data Table 2, supplementary information and patent number WO2017097792A1). TASK-1 inhibition by these blockers was voltage-independent and was not affected by high extracellular $\mathrm{K}^{+}$(Extended Data Fig. 5e-i). We assessed the 
selectivity of BAY1000493 against a range of diverse ion channels and found that it is highly selective for TASK-1 and TASK-3 (Extended Data Fig. 5b-d, Supplementary Table 1).

Crystal structures of complexes of TASK-1 with these inhibitors showed that they bind within the inner vestibule, directly below the selectivity filter (Fig 4c-f, Extended Data Fig. 6ag). In both cases, a single molecule binds across the 2-fold axis of the dimer, with one molecule bound per dimer. The electron density for BAY1000493 shows a superposition of two copies of the inhibitor, with 50\% occupancy for each orientation, confirmed by anomalous difference maps at the Br edge (Extended Data Fig. 6a,d,h). In contrast BAY2341237 binds 100\% in a single orientation, consistent with it inducing a small asymmetry in the dimer (Extended Data Fig. 6b,e,i and the Supplementary Information section).

Both inhibitors bind to the closed conformation of TASK-1, below the selectivity filter, without perturbing the X-gate structure. They fit precisely to the surface at the top of the vestibule, with Leu122 projecting into the vestibule below the compounds (Fig. 4e,f, Extended Data Fig. 6g). Both blockers form extensive hydrophobic interactions and van der Waals contacts with residues in the vestibule (Fig. 4e,f, Extended Data Fig. 6j,k). The complementarity between the inhibitor shapes and the upper vestibule is important for high affinity binding. Consistent with the interactions seen in the structures, mutation of residues lining the binding site (Thr93Cys, Ile118Ala, Leu122Ala, Thr199Cys, Leu239Ala and Asn240Ala) drastically reduced inhibition by BAY1000493 (Fig. 4g). Leu122 is important for binding of other TASK1 pore blockers ${ }^{26,29}$ and it occupies a key position below the bound inhibitors (Fig. 4e,f, Extended Data Fig. 6g). Full dose-response curves of TASK-1 and a Leu122Ala mutant, revealed a $>100$-fold decrease in $\mathrm{EC}_{50}$ values for both inhibitors with the mutation (Fig 4h,i).

Our structures show that both compounds bind at the top of the vestibule, trapped but not in direct contact with the X-gate. Leu244 is central to the X-gate structure, but is not part of the inhibitor binding site and a Leu244Ala mutation did not alter BAY1000493 inhibition (Fig. 4g, Extended Data Fig. 5a). However, TASK channels have another critical feature, their 
ability to retain bound compounds for long periods during washout experiments. BAY1000493 shows very slow washout rates from TASK-1 (Fig. 4j, Extended Data Fig. 5j), unlike a larger TASK inhibitor, A1899, which has an $\mathrm{EC}_{50}$ value of $35 \mathrm{nM}$ and washes off faster ${ }^{26}$ (Extended Data Fig. 5k-m). The X-gate and latch mutations Leu244Ala and Arg131Asp significantly increased the BAY1000493 washout rate (Fig. 4j,k, Extended Data Fig. 5j). Conversely, a mutation near the latch (Thr134Cys), that has relatively little effect on X-gate function (Fig. 3b), also has less effect on washout rates (Fig., 4j,k, Extended Data Fig. 5j). This suggests disruption of the X-gate or the latch may stabilise the TASK-1 open state, thereby allowing BAY1000493 to dissociate more easily.

The X-gate must open wide enough to allow both hydrated $\mathrm{K}^{+}$ions and the inhibitors to enter. Therefore, it is likely that X-gate opening requires a significant conformational change, not simply adjustments in sidechain positions or helix orientation. We speculate that this could involve opening of the latch, relaxation of the X-gate extended helix into an $\alpha$-helical conformation and straightening of M4 (Extended Data Fig. 7), giving a conformation similar to other $\mathrm{K}_{2 \mathrm{P}}$ structures ${ }^{17-21}$. This would allow the M1-M4 helices to adopt a more "up"-like conformation, without fenestrations (Extended Data Fig. 7), consistent with the higher activity states of other $\mathrm{K}_{2 \mathrm{PS}}$.

Loss-of-function missense mutations in TASK-1 cause autosomal dominant primary pulmonary hypertension Type $4^{30}$ (PPH4: OMIM: 615344), a condition that is often fatal in mid-life ${ }^{30}$, whilst TASK-3 mutations are linked to the developmental disorder Birk-Barel syndrome ${ }^{31}$. These mutations are distributed throughout the structures (see Extended Data Fig. 8a-g, Supplementary Discussion). One PPH4-associated TASK-1 mutation, Thr8Lys, is located on M1 within the X-gate latch and causes $~ 2$-fold decreased currents (Fig. 3a, Extended Data Fig 8c). Furthermore, two Birk-Barel syndrome TASK-3 mutations (Gly236Arg ${ }^{31}$ and Ala237Asp ${ }^{32}$ ) precede the hinge on M4, either inserting a large charged sidechain into the 
vestibule or next to the hinge (Extended Data Fig. 8h). These mutations emphasize the significance of these regions in TASK channel function.

In conclusion, we have shown that TASK channels are highly unusual $\mathrm{K}_{2 \mathrm{P}}$ channels in that they form a cytoplasmic X-gate, which may contribute to the low open probability of TASK-1. We have also shown that two nanomolar affinity inhibitors bind in the closed vestibule, trapped by the X-gate. Their precise fit at the top of the vestibule, combined with the trapping effect of the X-gate may explain the exceptionally high affinity and selectivity observed with these pore blockers. In addition, the presence of the X-gate may explain the very low wash-out rates of these inhibitors. Compound trapping within the inner cavity has been proposed previously for many ion channels ${ }^{33-35}$.

The X-gate is clearly important for the control of TASK channel activity and may contribute to their regulation by neurotransmitters ${ }^{13}$, intracellular signalling pathways ${ }^{13-15}$, volatile anaesthetics ${ }^{8-11,13}$ and small molecules ${ }^{26}$. However, given the polymodal nature of $\mathrm{K}_{2 \mathrm{P}}$ channel regulation and the known role of the selectivity filter in $\mathrm{K}_{2 \mathrm{P}}$ channel gating, the $\mathrm{X}$-gate is unlikely to be the only route for TASK channel regulation. For example, regulation by extracellular pH involves His98 and Gln209, both located in the extracellular region behind the selectivity filter. Although our data suggest that the $\mathrm{X}$-gate opening and the $\mathrm{pH}$ sensing are not tightly coupled, further studies may identify more subtle interactions between these gating mechanisms.

This study provides insight into the mechanism of TASK-1 inhibitors that are already in clinical trials as potential treatments for obstructive sleep apnea and atrial fibrillation. The inhibitor binding site within the vestibule, created by the closed X-gate, has significant implications for interpretation of the pharmacokinetic properties of TASK inhibitors. In particular, questions of bioavailability, metabolism and half-life should be interpreted in the light of the discovery of the X-gate. Once a blocker becomes sequestered within this vestibule, it will remain bound and 'active' for much longer than would be predicted by pharmacokinetic 
studies. Such factors should be carefully evaluated during drug development and trials. Overall, this work provides critical understanding of the gating mechanism in TASK channels and the action of clinically-relevant inhibitors, processes that are of uppermost importance for their use as targets for efficient and safe treatment of disease.

\section{References}

1 Karschin, C. et al. Expression pattern in brain of TASK-1, TASK-3, and a tandem pore domain $\mathrm{K}^{+}$channel subunit, TASK-5, associated with the central auditory nervous system. Mol Cell Neurosci 18, 632-648 (2001).

2 Jones, S. A. et al. Expression of TASK-1, a pH-sensitive twin-pore domain $\mathrm{K}^{+}$channel, in rat myocytes. Am J Physiol Heart Circ Physiol 283, H181-185 (2002).

3 Putzke, C. et al. The acid-sensitive potassium channel TASK-1 in rat cardiac muscle. Cardiovasc Res 75, 59-68 (2007).

4 Decher, N. et al. Knock-out of the potassium channel TASK-1 leads to a prolonged QT interval and a disturbed QRS complex. Cell Physiol Biochem 28, 77-86 (2011).

5 Olschewski, A. et al. Impact of TASK-1 in human pulmonary artery smooth muscle cells. Circ Res 98, 1072-1080 (2006).

6 Donner, B. C. et al. Functional role of TASK-1 in the heart: studies in TASK-1-deficient mice show prolonged cardiac repolarization and reduced heart rate variability. Basic Res Cardiol 106, 75-87 (2011).

7 Gurney, A. M. et al. Two-pore domain K channel, TASK-1, in pulmonary artery smooth muscle cells. Circ Res 93, 957-964 (2003).

8 Steinberg, E. A. et al. The role of $\mathrm{K}_{2 \mathrm{P}}$ channels in anaesthesia and sleep. Pflugers Arch 467, 907-916 (2015).

9 Putzke, C. et al. Differential effects of volatile and intravenous anesthetics on the activity of human TASK-1. Am J Physiol Cell Physiol 293, C1319-1326 (2007). 
10 Patel, A. J. et al. Inhalational anesthetics activate two-pore-domain background $\mathrm{K}^{+}$ channels. Nat Neurosci 2, 422-426 (1999).

11 Sirois, J. E. et al. The TASK-1 two-pore domain $\mathrm{K}^{+}$channel is a molecular substrate for neuronal effects of inhalation anesthetics. J Neurosci 20, 6347-6354 (2000).

12 Duprat, F. et al. TASK, a human background $\mathrm{K}^{+}$channel to sense external $\mathrm{pH}$ variations near physiological pH. The EMBO Journal 16, 5464-5471 (1997).

13 Talley, E. M. \& Bayliss, D. A. Modulation of TASK-1 (Kcnk3) and TASK-3 (Kcnk9) potassium channels: volatile anesthetics and neurotransmitters share a molecular site of action. J Biol Chem 277, 17733-17742 (2002).

14 Lopes, C. M. et al. PIP2 hydrolysis underlies agonist-induced inhibition and regulates voltage gating of two-pore domain $\mathrm{K}^{+}$channels. J Physiol 564, 117-129 (2005).

15 Wilke, B. U. et al. Diacylglycerol mediates regulation of TASK potassium channels by Gq-coupled receptors. Nat Commun 5, 5540 (2014).

16 Kiper, A. K. et al. Kv1.5 blockers preferentially inhibit TASK-1 channels: TASK-1 as a target against atrial fibrillation and obstructive sleep apnea? Pflugers Arch 467, 10811090 (2015).

17 Miller, A. N. \& Long, S. B. Crystal structure of the human two-pore domain potassium channel K $2 \mathrm{P} 1$. Science 335, 432-436 (2012).

18 Brohawn, S. G. et al. Crystal structure of the human K2P TRAAK, a lipid- and mechano-sensitive $\mathrm{K}^{+}$ion channel. Science 335, 436-441 (2012).

19 Brohawn, S. G. et al. Domain-swapped chain connectivity and gated membrane access in a Fab-mediated crystal of the human TRAAK K ${ }^{+}$channel. Proc Natl Acad Sci U S A 110, 2129-2134 (2013).

20 Dong, Y. Y. et al. K2P channel gating mechanisms revealed by structures of TREK-2 and a complex with Prozac. Science 347, 1256-1259 (2015). 
21 Lolicato, M. et al. $\mathrm{K}_{2 \mathrm{P}} 2.1$ (TREK-1)-activator complexes reveal a cryptic selectivity filter binding site. Nature 547, 364-368 (2017).

22 Morton, M. J. et al. Determinants of $\mathrm{pH}$ sensing in the two-pore domain $\mathrm{K}^{+}$channels TASK-1 and -2. Pflugers Arch 445, 577-583 (2003).

23 Stansfeld, P. J. et al. Insight into the mechanism of inactivation and $\mathrm{pH}$ sensitivity in potassium channels from molecular dynamics simulations. Biochemistry 47, 7414-7422 (2008).

24 Goldstein, M. et al. Functional mutagenesis screens reveal the 'cap structure' formation in disulfide-bridge free TASK channels. Scientific Reports 6, 19492 (2016).

25 Klesse, G. et al. CHAP: A Versatile Tool for the Structural and Functional Annotation of Ion Channel Pores. Journal of Molecular Biology 431, 3353-3365 (2019).

26 Streit, A. K. et al. A specific two-pore domain potassium channel blocker defines the structure of the TASK-1 open pore. J Biol Chem 286, 13977-13984 (2011).

27 Piechotta, P. L. et al. The pore structure and gating mechanism of K2P channels. EMBO J 30, 3607-3619 (2011).

28 Rinne, S. et al. TASK-1 and TASK-3 may form heterodimers in human atrial cardiomyocytes. J Mol Cell Cardiol 81, 71-80 (2015).

29 Chokshi, R. H. et al. Breathing Stimulant Compounds Inhibit TASK-3 Potassium Channel Function Likely by Binding at a Common Site in the Channel Pore. Mol Pharmacol 88, 926-934 (2015).

30 Ma, L. et al. A novel channelopathy in pulmonary arterial hypertension. $N$ Engl J Med 369, 351-361 (2013).

31 Barel, O. et al. Maternally inherited Birk Barel mental retardation dysmorphism syndrome caused by a mutation in the genomically imprinted potassium channel KCNK9. Am J Hum Genet 83, 193-199 (2008). 
32 Sediva, M. et al. Novel variant in the KCNK9 gene in a girl with Birk Barel syndrome. Eur J Med Genet (2019).

33 Armstrong, C. M. Interaction of tetraethylammonium ion derivatives with the potassium channels of giant axons. J Gen Physiol 58, 413-437 (1971).

34 Bolshakov, K. V. et al. Determinants of trapping block of N-methyl-d-aspartate receptor channels. J Neurochem 87, 56-65 (2003).

35 Linder, T. et al. Structural insights into trapping and dissociation of small molecules in $\mathrm{K}^{+}$channels. J Chem Inf Model 54, 3218-3228 (2014).

\section{Acknowledgements}

K.E.J.R. and D.S. are supported by B.B.S.R.C. grant no BB/N009274/1 to E.P.C. and S.J.T.. K.E.J.R., A.C.W.P., D.S., S.M.M.M., N.B.-B., E.P.C. are members of the SGC, a registered charity (number 1097737) that receives funds from AbbVie, Bayer Pharma AG, Boehringer Ingelheim, the Canada Foundation for Innovation, Genome Canada, Janssen, Merck KGaA, Merck \& Co., Novartis, the Ontario Ministry of Economic Development and Innovation, Pfizer, São Paulo Research Foundation-FAPESP and Takeda, as well as the Innovative Medicines Initiative Joint Undertaking ULTRA-DD grant 115766 and the Wellcome Trust (106169/Z/14/Z). L.J.C. is supported by a Wellcome Trust OXION PhD Studentship (109114/Z/15/Z). N.D. is supported by Deutsche Forschungsgemeinschaft (DFG) grant DE1482-4/1.

We thank the following: Diamond Light Source Ltd for access to the macromolecular crystallography beamlines, the Diamond Light Source staff for their help with data collection; all members of the SGC Biotech team, including Shayla Venkaya, Claire Strain-Damerell, Kasia Kupinska, Dong Wang and Katie Ellis; all members of the SGC IMP1 group, including Yin Yao Dong; David Eberhardt for help with the electrophysiology experiments; Rod Chalk, 
Tiago Moreira, Georgina Berridge and Octawia Borkowska for help with mass spectrometry; Brian Marsden and David Damerell, James Bray, James Crowe and Chris Sluman for bioinformatics support; Frank von Delft, Tobias Krojer and Beth MacLean for assistance with crystallography infrastructure; Oxana Nowak for technical assistance and Thomas Baukrowitz (Christian Albrechts University of Kiel, Kiel, Germany) for critical reading of the manuscript.

\section{Author Contributions}

K.E.J.R. and A.K.K, contributed equally to the project and should be considered as joint first authors. W.Z., S.R. and A.C.W.P. contributed equally to the project and should be considered as join second authors. The project was conceived and managed by E.P.C., N.D. and T.M.. K.E.J.R., obtained crystals that diffracted to beyond $4 \AA$ resolution, collected data and solved the structure of TASK-1 and the complexes with drug molecules, assisted by D.S., and supervised by A.C.W.P. and E.P.C.. W.Z. was involved in optimization of the constructs, protein purification and production of initial crystals, supervised by A.Q., A.C.W.P. and E.P.C.. A.K.K. performed voltage clamp recordings, pharmacological experiments and single channel recordings and S.R. performed voltage clamp recordings, introduced all TASK-1 mutants and performed ELISA surface expression assays, M.G. performed all experiments with sevoflurane, all these experiments were supervised by N.D.. Initial constructs for structural biology were screened for expression by L.S. and large scale insect cell expressions were produced by S.M.M.M., supervised by N.B.B.. Tests of the relative activities of TASK-1 and the TASK$1^{\text {Met1-Glu259 }}$ construct used in crystallisation were performed by L.J.C., supervised by S.J.T.. The TASK-1 inhibitors were designed and produced by M.D., M.H., H.M and T.M., supervised by T.M.. Data were analyzed and the paper was written by K.E.J.R., A.C.W.P., A.K.K., S.R., L.J.C., S.J.T., T.M., N.D. and E.P.C.. 
Figures

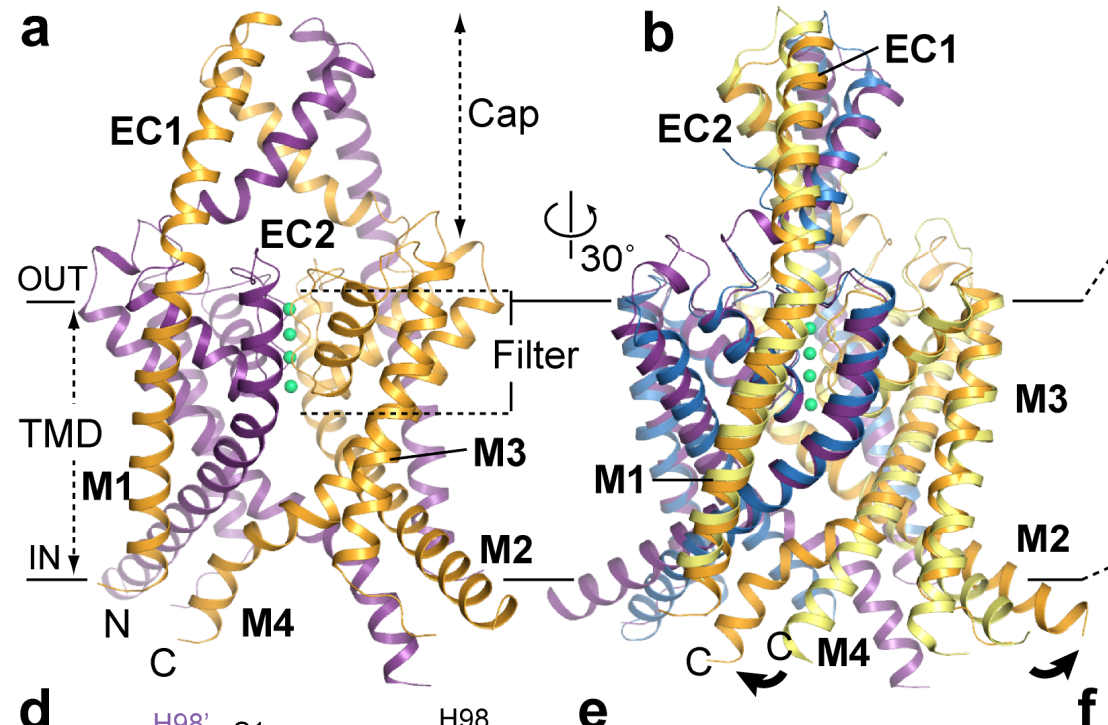

C Pore radius $(\AA)$
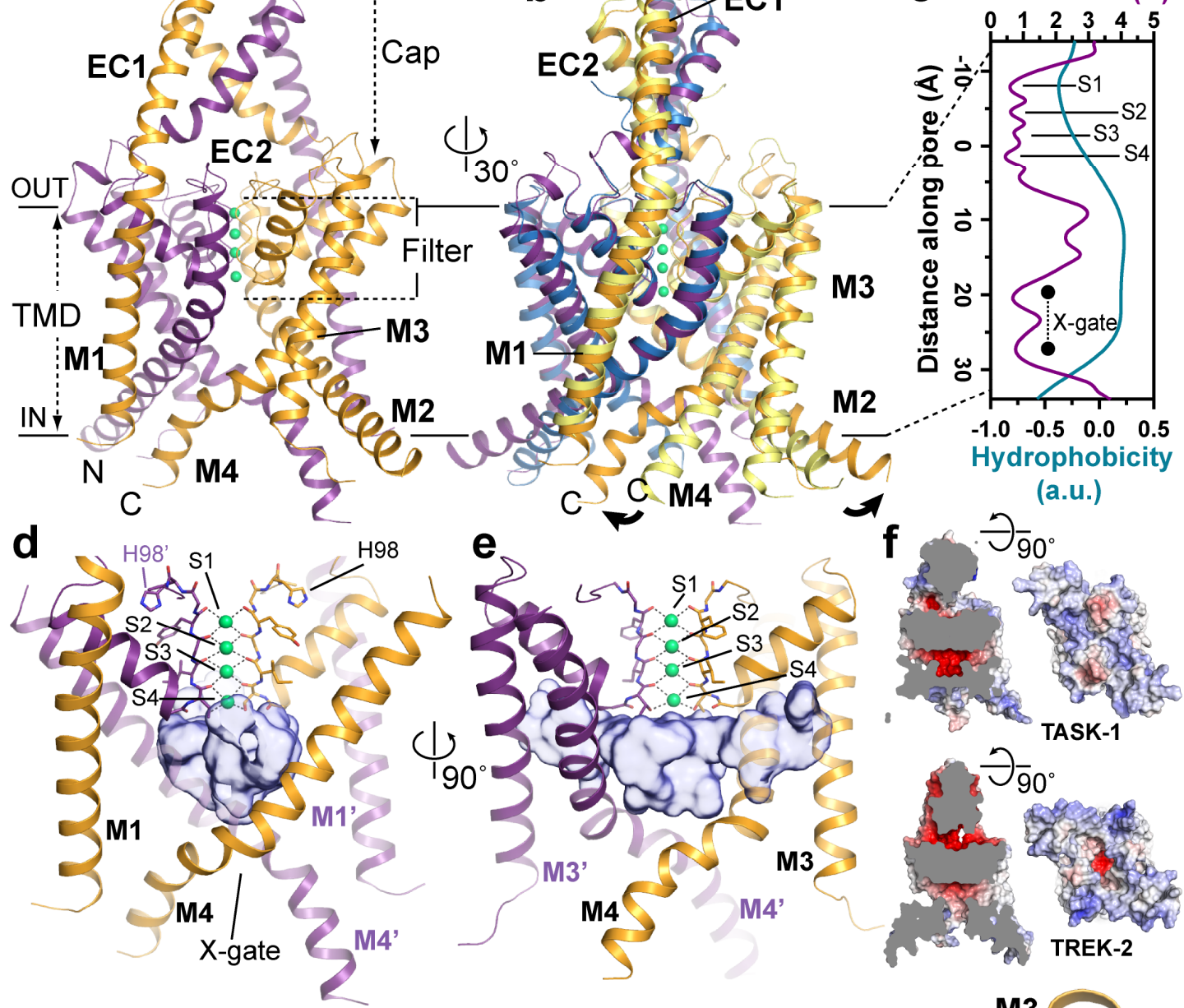

(a.u.)

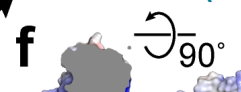

g
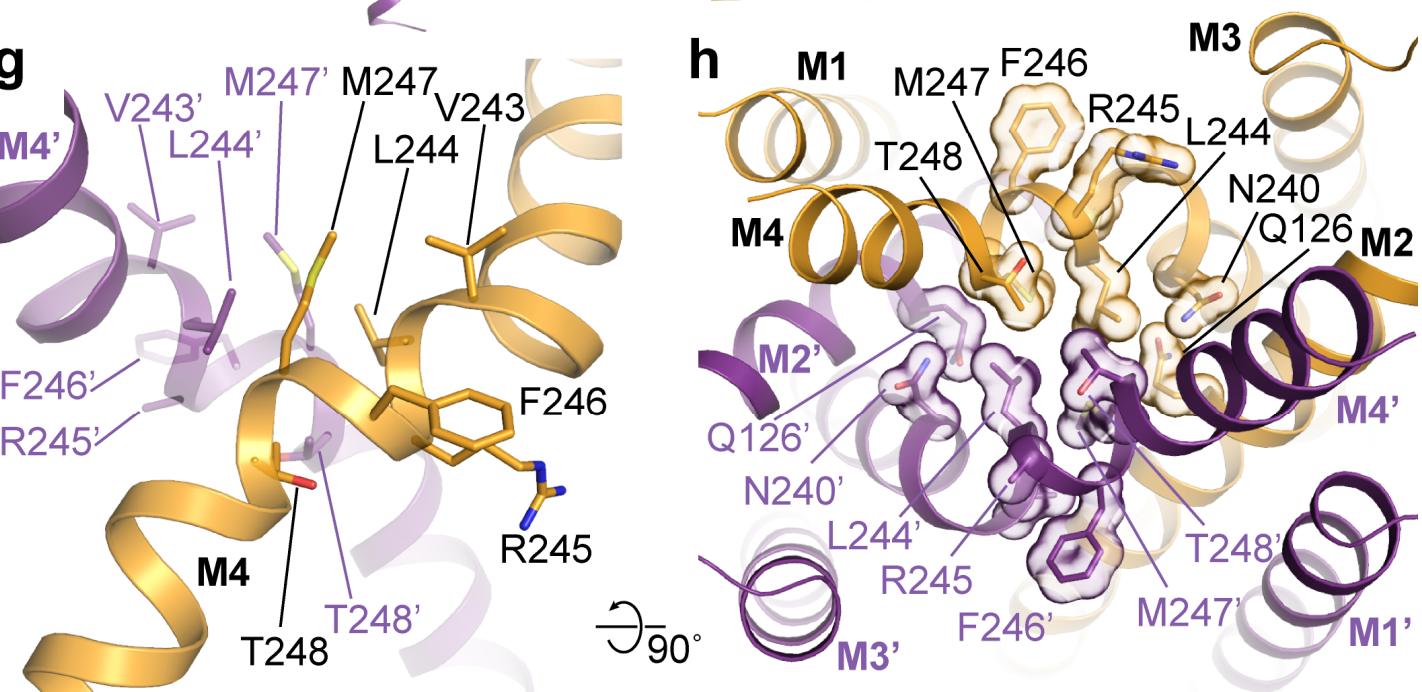

Fig. 1 | The structure of TASK-1 reveals a unique crossed helix X-gate. a, TASK-1 structure cartoon, with chain A (gold), B (purple) and potassium ions (green), viewed from the membrane. b, Superposition of the TASK-1 (gold/purple) and TREK-2 ( "down" state (PDB: 4XDJ) (yellow/blue) structures. c, Radius and hydrophobicity of the TASK-1 pore ${ }^{25}$. The X- 
gate enclosing the vestibule and fenestrations (depicted with semi-transparent surfaces), d, viewed from the membrane plane and e, rotated $90^{\circ}$. f, Electrostatic surfaces of TASK-1 and TREK-2 (PDB: 4XDJ) shown as a cross section perpendicular to membrane and seen from below. The X-gate viewed from $\mathbf{g}$, the plane of the membrane and $\mathbf{h}$, below the membrane. 

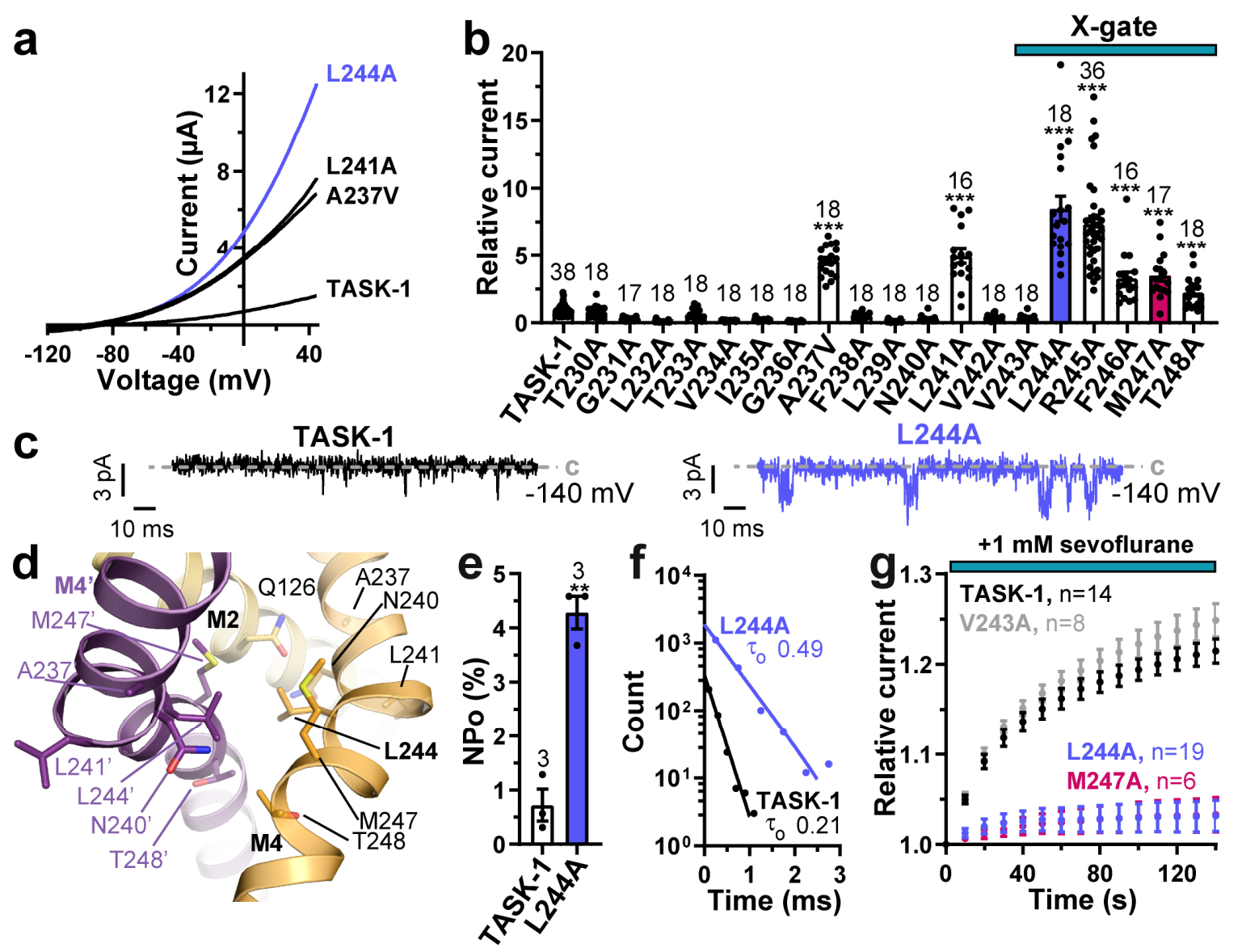

Fig. 2 Mutation of X-gate residues results in an increase in ion conduction and loss of anaesthetic activation. a, Representative two-electrode voltage-clamp recordings of TASK-1 wild-type $(\mathrm{n}=38)$ and X-gate mutants Ala237Val $(\mathrm{n}=18)$, Leu241Ala $(\mathrm{n}=16)$ and Leu244Ala $(\mathrm{n}=18)$. b, Relative current amplitudes of wild-type and mutated TASK-1 (two-sided Mood's median test, $P_{\mathrm{A} 237 \mathrm{~V}}=2.6 \mathrm{E}-07, P_{\mathrm{L} 241 \mathrm{~A}}=1.9 \mathrm{E}-06, P_{\mathrm{L} 244 \mathrm{~A}}=2.6 \mathrm{E}-07, P_{\mathrm{F} 246 \mathrm{~A}}=1.9 \mathrm{E}-06, P_{\mathrm{M} 247 \mathrm{~A}}=7.9 \mathrm{E}-$ 06, $P_{\mathrm{T} 248 \mathrm{~A}}=6.0 \mathrm{E}-04$; using two-sided Mann-Whitney U test $\left.P_{\mathrm{R} 245 \mathrm{~A}}=1.5 \mathrm{E}-13\right)$. c, Representative inside-out single channel recordings of TASK-1 wild-type (n=3) and Leu244Ala (n=3). d, The $\mathrm{X}$-gate viewed from the membrane plane, rotated $90^{\circ}$ relative to Fig. $1 \mathrm{~g}$ showing Leu244 and surrounding residues. Analysis of e, the open probability (NPo) (two-sided Mood's median test, $P=1.4 \mathrm{E}-02)$ and $\mathbf{f}$, the mean open times. $\mathbf{g}$, Application of $1 \mathrm{mM}$ sevoflurane to wild-type $(n=14)$, Val243Ala (n=8), Leu244Ala (n=19) and Met247Ala (n=6). All two-electrode voltageclamp recordings and single channel measurements in Fig. 2-4 were performed in X. laevis oocytes. Number of biological replicates (n) are illustrated in the respective figure legends or graphs. Panels b,e,g data are presented as mean \pm s.e.m.. n.s., not significant; **, $P<0.01$; ***, $P<0.001$. For full statistical details see Methods and Source Data File. 

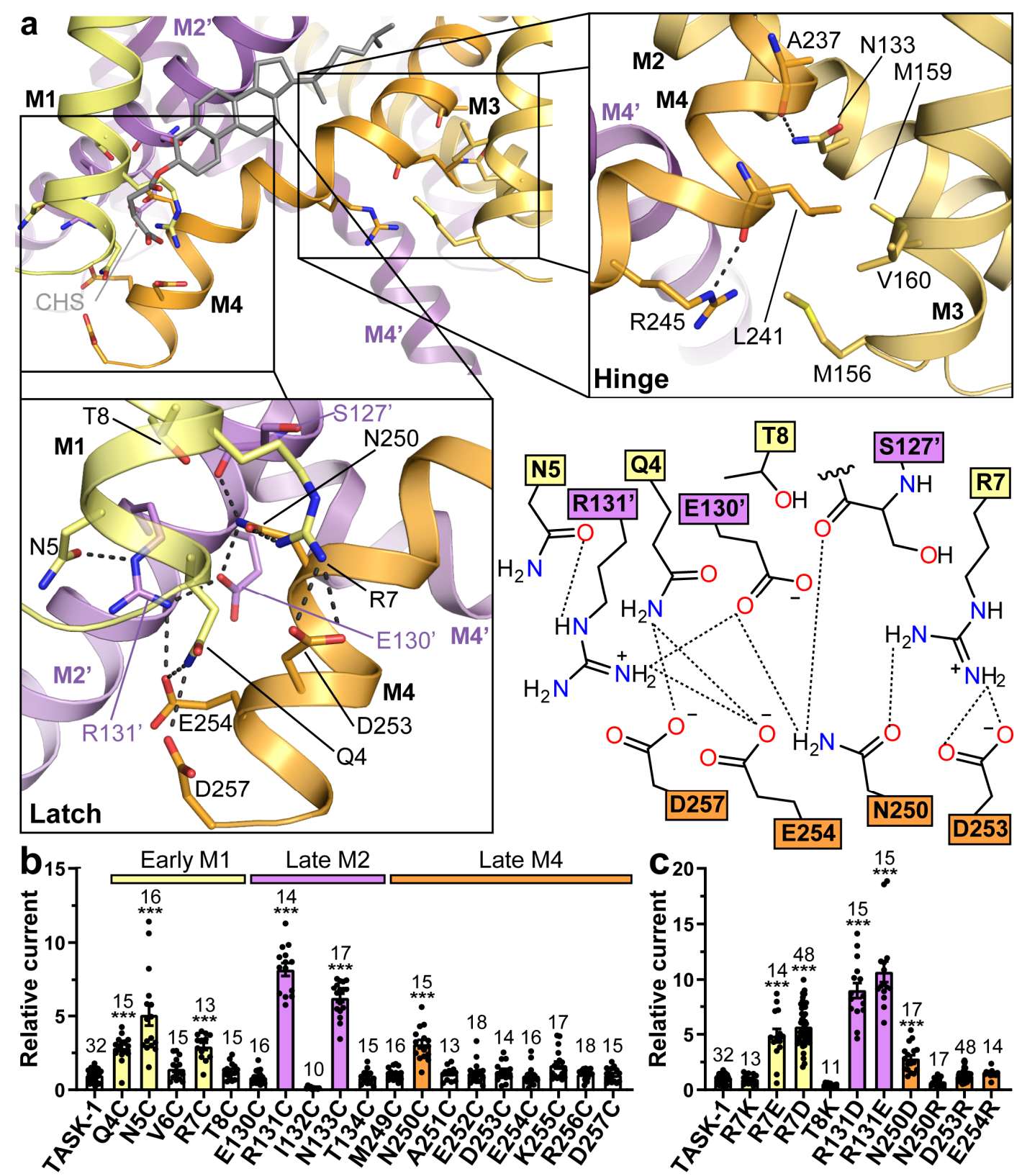

Fig. 3 The $X$-gate environment includes a hinge and a latch on either side of the vestibule which are required for $\mathrm{X}$-gate integrity. a, The X-gate in TASK-1, with the cholesteryl hemisuccinate (CHS) binding site. The hinge is shown on the right and the latch region below. b, Relative current amplitudes of wild-type and mutated TASK-1 (two-sided Welch's t-test, $P_{\mathrm{Q} 4 \mathrm{C}}=6.6 \mathrm{E}-07, P_{\mathrm{R} 7 \mathrm{C}}=2.5 \mathrm{E}-06, P_{\mathrm{R} 131 \mathrm{C}}=2.5 \mathrm{E}-10, P_{\mathrm{N} 133 \mathrm{C}}=9.4 \mathrm{E}-13, P_{\mathrm{N} 250 \mathrm{C}}=3.1 \mathrm{E}-06 ;$ using twosided Mood's median test $\left.P_{\mathrm{N} 5 \mathrm{C}}=9.6 \mathrm{E}-07\right)$. c, Relative current amplitudes for mutated residues in the latch (two-sided Welch's t-test, $P_{\mathrm{R} 7 \mathrm{E}}=2.5 \mathrm{E}-05, P_{\mathrm{R} 131 \mathrm{D}}=1.7 \mathrm{E}-08, P_{\mathrm{N} 250 \mathrm{D}}=1.2 \mathrm{E}-05$; twosided Mood's median test $\left.P_{\mathrm{R} 7 \mathrm{D}}=2.8 \mathrm{E}-13, P_{\mathrm{R} 131 \mathrm{E}}=1.6 \mathrm{E}-06\right)$. Number of biological replicates (n) are illustrated in the respective figure legends or graphs. b,c, Data are presented as mean \pm s.e.m.. ***, $P<0.001$. For full statistical details see Methods and Source Data File. 


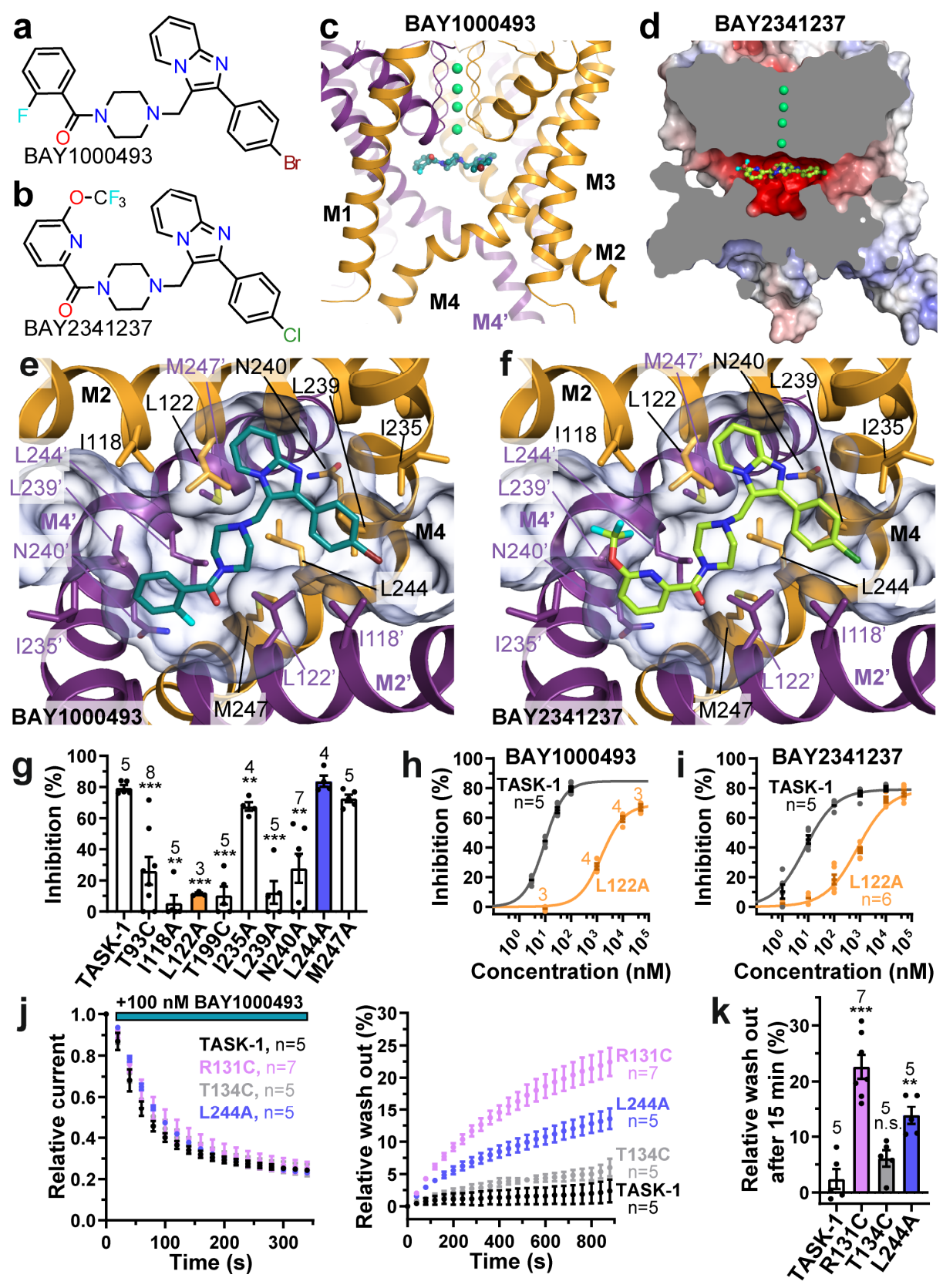

Fig. 4 | Structures of complexes with two highly potent inhibitors show binding in the vestibule, trapped by the X-gate. Chemical structures of a, BAY1000493 ((4-\{[2-(4bromophenyl)imidazo[1,2-a]pyridin-3-yl]methyl \} piperazin-1-yl)(2-fluorophenyl)methanone) and b, BAY2341237 ((4-\{[2-(4-chlorophenyl)imidazo[1,2-a]pyridin-3-yl]methyl \}piperazin-1yl)[6-(trifluoromethoxy)pyridin-2-yl]methanone). c, Structure of the TASK-1/BAY1000493 (teal) complex (M2' excluded). For simplicity, only one orientation of BAY1000493 is shown, although it was modelled in two conformations (see Extended Data Fig. 6a). d, Cross section of an electrostatic surface of the TASK-1/BAY2341237 (green) complex. e, The BAY1000493 and f, BAY2341237 binding sites below the selectivity filter, looking towards the X-gate with contoured surfaces showing the vestibule surface in grey. g, Percentage of inhibition of wild- 
type and mutated TASK-1 by $100 \mathrm{nM}$ BAY1000493 (two-sided Student's t-test, $P_{\mathrm{T} 93 \mathrm{C}}=7.8 \mathrm{E}-$ 04, $P_{\mathrm{L} 122 \mathrm{~A}}=1.6 \mathrm{E}-07, P_{\mathrm{T} 199 \mathrm{C}}=3.0 \mathrm{E}-06, P_{\mathrm{I} 235 \mathrm{~A}}=7.3 \mathrm{E}-03, P_{\mathrm{L} 239 \mathrm{~A}}=1.9 \mathrm{E}-05, P_{\mathrm{N} 240 \mathrm{~A}}=1.0 \mathrm{E}-3$; two sided Mann-Whitney U test $\left.P_{\mathrm{I} 118 \mathrm{~A}}=2.0 \mathrm{E}-02\right)$. Dose-response curves for $\mathbf{h}, \mathrm{BAY} 1000493$ and $\mathbf{i}$, BAY2341237 for wild-type TASK-1 and Leu122Ala. j, Analysis of relative current amplitudes over time while washing BAY1000493 in and out. k, Analysis of the relative wash-out (using two-sided Student's t-test, $\left.P_{\mathrm{T} 134 \mathrm{C}}=1.5 \mathrm{E}-01, P_{\mathrm{L} 244 \mathrm{~A}}=1.4 \mathrm{E}-3, P_{\mathrm{R} 131 \mathrm{C}}=4.9 \mathrm{E}-5\right)$. Number of biological replicates (n) are illustrated in the respective graphs. Data in $\mathbf{g - k}$, are presented as mean \pm s.e.m.. n.s., not significant; $* *, P<0.01$; ***, $P<0.001$. For full statistical details see Methods and Source Data File. 2Fo-Fc and Fo-Fc electron density maps are shown in Extended Data Fig. 6. 


\section{Methods}

\section{Cloning and expression of TASK-1 ${ }^{\text {Met1-Glu259 }}$ for structural studies}

The human KCNK3 gene (Genbank ID 4504849), which encoded the TASK-1 protein, was obtained from Origene. The TASK-1 construct, residues Met1 to Glu259, was subcloned in the SGC vector pFB-CT10HF-LIC, with a C terminal tobacco etch virus (TEV) protease site followed by decahistidine and FLAG tags. Baculovirus was generated with the Bac-to-bac system and passaged twice before it was used for infecting Spodoptera frugiperda (Sf9) cells (Thermo-Fisher Scientific, Cat. No. 11496015) at a density of $2 \times 10^{6}$ cells $/ \mathrm{mL}$ and a ratio of 5 $\mathrm{mL}$ virus per litre cells. Infected cells were harvested after $72 \mathrm{~h}$ by centrifugation, frozen in liquid nitrogen and stored at $-80^{\circ} \mathrm{C}$.

\section{Purification of TASK-1}

Cells were broken with an EmulsiFlex-C3 or C5 high-pressure homogenizer (Avestin Inc.) in $40 \mathrm{ml}$ breaking buffer (50 mM HEPES pH 7.5, $200 \mathrm{mM} \mathrm{KCl,} \mathrm{5 \%} \mathrm{v/v} \mathrm{glycerol)} \mathrm{per}$ initial litre culture volume. Protein was solubilized by addition of $1 \% \mathrm{w} / \mathrm{v}$ n-decyl- $\beta$-Dmaltopyranoside (DM) (Anatrace) and $0.1 \% \mathrm{w} / \mathrm{v}$ cholesteryl hemisuccinate (CHS) Tris salt (Sigma-Aldrich) and rotated at $4^{\circ} \mathrm{C}$ for $1 \mathrm{~h}$. Cell debris was pelleted at $35,000 \mathrm{~g}, 1 \mathrm{~h}, 4^{\circ} \mathrm{C}, 5$ $\mathrm{mM}$ imidazole $\mathrm{pH} 8.0$ was added, and protein was incubated with Talon (Clontech), $0.5 \mathrm{ml}$ resin per litre cell culture for $1 \mathrm{~h}$ at $4^{\circ} \mathrm{C}$. Resin was collected and washed with $30 \mathrm{CV}$ wash buffer (50 mM HEPES pH 7.5, $200 \mathrm{mM} \mathrm{KCl,} \mathrm{5 \%} \mathrm{v/v} \mathrm{glycerol,} 20 \mathrm{mM}$ imidazole pH 8.0, 0.24\% w/v DM, 0.024\% w/v CHS), and eluted with elution buffer (50 mM HEPES pH 7.5, $200 \mathrm{mM}$ $\mathrm{KCl}, 5 \% \mathrm{v} / \mathrm{v}$ glycerol, $250 \mathrm{mM}$ imidazole $\mathrm{pH} 8.0,0.24 \% \mathrm{w} / \mathrm{v} \mathrm{DM}, 0.024 \% \mathrm{w} / \mathrm{v}$ CHS). The protein was exchanged into desalting buffer (50 mM HEPES pH 7.5, $200 \mathrm{mM} \mathrm{KCl,} \mathrm{5 \%} \mathrm{v/v}$ glycerol, $0.24 \%$ w/v DM, 0.024\% w/v CHS) by passing over PD10 columns and purification tags were cleaved by addition of a 1:3 w:w ratio of $6 \mathrm{x}$ His-tagged TEV protease and deglycosylated with a 1:10 w:w ratio of $6 \mathrm{x}$ His-tagged PNGaseF, for 12-16 h at $4^{\circ} \mathrm{C}$. Enzymes were removed by addition of $0.1 \mathrm{ml}$ Talon resin per litre initial cell culture and $7.5 \mathrm{mM}$ 
imidazole $\mathrm{pH}$ 8.0. The protein was concentrated to $500 \mu \mathrm{l}$ and subjected to size exclusion chromatography on a Superose 6 Increase 10/300 GL column (GE Healthcare) in gel filtration buffer (20 mM HEPES pH 7.5, $200 \mathrm{mM} \mathrm{KCl,} \mathrm{0.12 \%} \mathrm{w/v} \mathrm{DM,} \mathrm{0.012 \%} \mathrm{w/v} \mathrm{CHS).} \mathrm{Fractions}$ containing the highest concentration of TASK-1 were pooled and concentrated to $12-30 \mathrm{mg} / \mathrm{ml}$ $\mathrm{ml}$ with a $50 \mathrm{kDa}$ molecular weight cut off spin concentrator (GE Healthcare).

\section{Crystallisation, X-ray data collection and data processing}

Crystallization trials were performed in 96-well sitting drop vapour diffusion plates using a Mosquito crystallization robot (TTP Labtech) at 8-12 mg/ml with $150 \mathrm{nl}$ drops and 2:1, 1:1 and 1:2 protein:reservoir ratios. Initial crystals were obtained at $20^{\circ} \mathrm{C}$ in an in-house version of MemGold HT-96, condition D7 (0.1 M TRIS pH 8.5, $0.1 \mathrm{mM} \mathrm{KCl}$ and 39\% v/v polyethylene glycol (PEG) 400). Crystals were further optimized by addition of $3 \% \mathrm{w} / \mathrm{v}$ sucrose to the precipitant solution and by using the HiLiDe method ${ }^{36}$. Briefly, 1,2-dioleoyl-sn-glycero-3phosphocholine (DOPC) (Avanti Polar Lipids, Inc.) in chloroform was dried down in a roundbottomed glass vial, $1.5 \mu \mathrm{g}$ lipid per $\mu 1$ protein. Protein at $5.7-6.2 \mathrm{mg} / \mathrm{ml}$ was added along with $15 \mu \mathrm{g} / \mu \mathrm{l} \mathrm{DM}$ and $1.5 \mu \mathrm{g} / \mu \mathrm{l} \mathrm{CHS}$, incubated slowly shaking at $4^{\circ} \mathrm{C}$ for $16-24 \mathrm{~h}$ and centrifuged at $15,000 \mathrm{~g}, 2 \mathrm{~h}, 4^{\circ} \mathrm{C}$. Crystallization was performed in 24-well hanging drop plates with $2 \mu 1$ drops and a 2:1 protein:reservoir ratio at $20^{\circ} \mathrm{C}$. Crystals grew over 1-8 weeks in $0.1 \mathrm{M}$ TRIS $\mathrm{pH} 8.5,0.05 \mathrm{M} \mathrm{KCl}, 32 \%$ PEG 400 and $3 \% \mathrm{w} / \mathrm{v}$ sucrose and were harvested at $6^{\circ} \mathrm{C}$ directly from the drop and vitrified.

Data were collected at Diamond Light Source Ltd. (DLS) using the microfocus beamline I24. The final dataset was assembled from a series of $40^{\circ}$ wedges collected from two crystals, using a 9x6 $\mu \mathrm{m}$ microbeam.

Crystals of complexes of TASK-1 with inhibitors were also grown using the HiLiDe method. Each inhibitor, dissolved in 100\% DMSO at $130 \mathrm{mM}$, was added to the HiLiDe setup to a final concentration of $1.3 \mathrm{mM}$. Crystals grew in $0.1 \mathrm{M}$ TRIS pH 8.5, $0.05 \mathrm{M} \mathrm{KCl}$, and 3\% 
w/v sucrose with 24\% PEG 400 for BAY1000493 and 31\% PEG 400 for BAY2341237 over 1

- 12 weeks and were harvested at $6^{\circ} \mathrm{C}$. Data were collected at the DLS on beamline I24 at a wavelength of $0.9686 \AA$, with $0.1 \mathrm{~s}$ exposures, a beam size of $20 \mathrm{x} 20 \mu \mathrm{m}$ and $0.2^{\circ}$ oscillations. A full dataset for the BAY1000493 complex was assembled from several $40^{\circ}$ wedges from one crystal whereas the BAY2341237 dataset was collected as a single wedge, both with a 20x20 $\mu \mathrm{m}$ beam. Anomalous data for BAY1000493 were collected at the bromine edge, $(\lambda=0.9116$ $\AA$ ) in four $360^{\circ}$ passes.

\section{Model building and refinement for structures}

Data were processed as individual wedges in XDS (version Jan 26 2018) ${ }^{37}$ and scaled together in XSCALE (versions Jan 262018 or Mar 15 2019) ${ }^{37}$. The data were merged in AIMLESS (version 0.7.3) within CCP4i (version 7.0) ${ }^{38,39}$ (Extended Data Table 1). Due to the anisotropy of the data, an anisotropic cut-off was applied using the STARANISO server (versions 2.2.12 and 2.2.19) ${ }^{40}$. The structure was solved using molecular replacement with Phaser (version 2.7.17) ${ }^{41}$ using a truncated model of TREK-2 ${ }^{20}$ (PDB ID: 4BW5) with separate search models consisting of the TM domain and the cap. Two copies of the TASK-1 dimer were located in the asymmetric unit (Extended Data Fig. 2k). An initial TASK-1 model was built using a density modified prime-and-switch map calculated using phenix.autobuild (version $1.12)^{42}$ as a guide and was improved by several rounds of manual model building and refinement in Coot (version 0.8.9.2) ${ }^{43}$. Refinement was carried out in BUSTER (version 2.10.3 $)^{44}$, using all data to $3.0 \AA$ in the highest resolved direction, with NCS restraints and one TLS group per chain. Four potassium ions per homodimer were modelled and distances from the oxygen atoms in the filter were restrained to $2.8 \AA$. The final model for the native structure comprised residues Met1 to Asp257 in chains A and C, and Met1 to Leu261 in chains B and D. In the $\mathrm{AB}$ dimer, residues 149 to 151 are disordered and were not modelled. The selectivity filter of TASK-1 consists of residues $\mathrm{T}^{93} \mathrm{IGY}^{96}$ and $\mathrm{T}^{199} \mathrm{IGF}^{202}$ from each subunit, and they 
adopt the expected conformation seen in other $\mathrm{K}_{2 \mathrm{P}}$ structures ${ }^{17-21}$. The carbonyl oxygens of these residues, as well as the sidechain oxygens of Thr93 and $\mathrm{Thr} 199$, coordinate four $\mathrm{K}^{+}$ions in sites S1-S4 (Fig. 1c-e, Extended Data Fig. 2b). However, there is weaker density for the S2 $\mathrm{K}^{+}$ion and it refines to have a higher $\mathrm{B}$ factor in all structures, compared to the three other $\mathrm{K}^{+}$ ions. Omitting this $\mathrm{K}^{+}$ion altogether resulted in a positive Fo-Fc peak, so it was included in the model, with $100 \%$ occupancy and a higher B factor. Ramachandran statistics for the final model were $97.74 \%$ in favoured and $2.26 \%$ in allowed regions.

The structure contains a well-defined CHS molecule packed against the X-gate. It lies in an extensive, largely hydrophobic groove formed by residues Arg3, Arg7, Pro119, Val123, Val243, Phe246 and Met249. The two arginine residues are adjacent to the hemisuccinate moiety whereas the hydrophobic residues are adjacent to the cholesterol unit.

Data for the ligand complexes were processed in the same way as the native complex (Extended Data Table 1) to $2.9 \AA$ And 3.1 $\AA$ for BAY1000493 and BAY2341237, respectively. After one round of refinement in BUSTER (version 2.10.3) ${ }^{44}$, positive difference density was apparent in the vestibule. The BAY1000493 data collected at the bromine edge was processed as described previously and an anomalous difference map was calculated in phenix.find_peaks_holes (version 1.15$)^{42,45}$, revealing two $\mathrm{Br}$ peaks per dimer. Since BAY1000493 binds across the dimer axis below the selectivity filter, only one copy of the inhibitor is bound per TASK-1 dimer and it always binds in the same position. In the case of BAY1000493, the inhibitor binds in either orientation with a 50\% probability, so the density in the pore is the average of the two possible orientations throughout the crystal. However, each dimer has one molecule of BAY1000493 bound and it only binds in one position, with the same interactions regardless of the orientation (Supplementary Information and Extended Data Fig. $\mathrm{a}, \mathrm{h})$.

In contrast, The Fo-Fc difference density map calculated with BAY2341237 omitted from the structure, revealed a single orientation for the compound, which was modelled with 
100\% occupancy (Extended Data Fig. 6b,e,i and Supplementary Information for further explanation). The final models were refined against the anisotropically truncated STARANISO $^{40}$ data, with Ramachandran statistics of $97.56 \%$ in allowed and $2.44 \%$ in preferred regions and $97.54 \%$ in allowed and $2.46 \%$ in preferred, for the BAY1000493 and BAY2341237 complexes, respectively.

\section{Assessment of the sizes of spheres that can enter the vestibule from the cytoplasm}

To assess the size of sphere that could pass through the lower gate we used CHAP (version 0.9 .1$)^{25}$. The X-gate has a narrow gap between the M4 helices, allowing spheres of no more than $0.8 \AA$ radius to pass through the gate between the C $\delta 1$ atoms of Leu244 and Leu244', and the S $\delta$ atom of Met247 (Fig. 1e,f), in contrast to TREK-2 (PDB:4XDJ) which would allow spheres of $7.5 \AA$ radius to pass between the $\mathrm{O} \delta 1$ atoms of Asp325 and Asp325', and the C $\beta$ and C $\gamma$ atoms of $\operatorname{Arg} 328$.

\section{Two-electrode voltage-clamp measurements}

The frogs from which we obtained oocytes were adult, sexually mature, female Xenopus laevis (Nasco®, Fort Atkinson, USA). This study conforms to the guide for the Care and Use of laboratory Animals (NIH Publication 85-23). Experiments using Xenopus frogs were approved by the local ethics commission of the "Regierungspräsidium Giessen" (MR 20/28 Nr. A 23/2017). X. laevis oocytes were obtained and the TEVC measurements were recorded as described previously ${ }^{26}$. Briefly, collected oocytes were stored at $18{ }^{\circ} \mathrm{C}$ in ND96 solution (in mM: $\mathrm{NaCl} 96, \mathrm{KCl} 2, \mathrm{CaCl}_{2} 1.8, \mathrm{MgCl}_{2}$ 1, HEPES 5 and $\left.\mathrm{pH} 7.5\right)$ supplemented with $50 \mathrm{mg} / \mathrm{l}$ gentamycin, $274 \mathrm{mg} / \mathrm{l}$ sodium pyruvate and $88 \mathrm{mg} / \mathrm{l}$ theophylline. Oocytes were injected with $5 \mathrm{ng}$ of TASK-1 cRNA and incubated for $48 \mathrm{~h}$ at $18{ }^{\circ} \mathrm{C}$. ND96 was used as recording solution. Oocytes were held at $-80 \mathrm{mV}$ and voltage was ramped from -120 to $+45 \mathrm{mV}$ within $3.5 \mathrm{~s}$, using a sweep time interval of $4 \mathrm{~s}$. Block was analysed with voltage steps from a holding potential of $-80 \mathrm{mV}$. A first test pulse to $0 \mathrm{mV}$ of $1 \mathrm{~s}$ duration was followed by a repolarising step to -80 
$\mathrm{mV}$ for $1 \mathrm{~s}$, directly followed by another $1 \mathrm{~s}$ test pulse to $+40 \mathrm{mV}$. The sweep time interval was 10 s. All inhibitors were dissolved in DMSO, aliquoted, stored at $-20{ }^{\circ} \mathrm{C}$ or room temperature and added to the external solution (ND96) just before the recordings. The $\mathrm{EC}_{50}$ was determined from Hill plots using four concentrations for each construct. Final DMSO concentration of 0.1 $\%$ was not exceed.

For measurements of the TASK-1 $1^{\text {Met1-Glu259 }}$ currents shown in the Extended Data Fig. 1a-c oocytes were injected with $1 \mathrm{ng}$ of TASK-1 RNA and two-electrode voltage-clamp experiments performed with a Warner oocyte-clamp (OC-725-C). Data were digitalised with an Digidata1400A interface (Molecular Devices). Voltage-ramp protocols were used to measure IV-responses. The ramps lasted for $0.5 \mathrm{~s}$ with ranges of $-120 \mathrm{mV}$ to $50 \mathrm{mV}$. In each experimental condition 10 ramps were recorded with the last 5 sweeps of such a series averaged and used for analysis. Voltage-clamp recordings were performed in ND96 solution adjusted to the desired $\mathrm{pH}$ at room temperature with $\mathrm{NaOH}$. For experiments at $\mathrm{pH}$ 5.5, HEPES was exchanged for MES. Data were analysed with ClampFit10 (Molecular Devices), Excel 2013 (Microsoft) and Origin 2016 (OriginLab Corporation).

\section{Chemiluminescence assay in $X$. laevis oocytes}

Surface expression of TASK-1 channel constructs was studied as previously described $^{46}$. Briefly, X. laevis oocytes were injected with cRNA of HA-tagged channels. After 48h, oocytes were incubated in ND96 plus $1 \%$ BSA on ice for 30 min to reduce unspecific antibody binding. Subsequently, the oocytes were incubated with primary antibody (rat antiHA (Roche), 1:100) for $1 \mathrm{~h}$, and after extensive washing they were incubated with secondary antibody (goat anti-rat-IgG, HRP-coupled (Dianova), 1:500) for $1 \mathrm{~h}$. After oocytes were individually placed into a vial with $20 \mu \mathrm{l}$ of luminescence substrate (SuperSignal Femto (Thermo Scientific)), light emission was detected with a GloMax luminometer (Promega). 


\section{Macropatch clamp and single channel measurements}

Inside-out single channel patch clamp recordings of Xenopus oocytes were performed similar as previously described ${ }^{28}$. Macropatch clamp and single channel current recordings were executed at room temperature with an Axopatch 200B amplifier (Axon Instruments), a Digidata 1550B A/D converter (Axon Instruments) and pClamp10 software (Axon Instruments). The sampling rate was $15 \mathrm{kHz}$ with the analog filter set to $2 \mathrm{kHz}$. For single channel recordings pipette and bath solution contained in mM: KCl 140, HEPES 5, EGTA 1 and pH 7.4. Voltage pulses were applied from $0 \mathrm{mV}$ (holding potential) to $+140 \mathrm{mV}$ and/or $-140 \mathrm{mV}$ for $1 \mathrm{~s}$ with an interpulse interval of $8 \mathrm{~s}$. For inside-out macropatch clamp recordings the pipette solution contained in $\mathrm{mM}$ : $\mathrm{NaCl} 114, \mathrm{KCl} 6, \mathrm{HEPES} 10, \mathrm{CaCl}_{2} 3.6$ and $\mathrm{pH}$ 7.4. The bath solution contained in mM: KCl 120, HEPES 10, EGTA 2, Na4-pyrophosphate 1 and pH 7.4. Voltage pulses were applied from $-80 \mathrm{mV}$ to $+60 \mathrm{mV}$ for $1 \mathrm{~s}$ with an interpulse interval of $0.3 \mathrm{~s}$. CHS was prepared as a $125 \mathrm{mM}$ stock solution in DMSO and freshly added to the bath solution before the recordings. CHS and TEA-Cl (Sigma Aldrich) were applied with a HSSE-2/3 rapid perfusion system (ALA Scientific Instruments) to the intracellular phase of the lipid membrane. Data were analysed with ClampFit10 (Molecular Devices), Excel 2013 (Microsoft) and Origin 2016 (OriginLab Corporation).

\section{Quantification and statistical analysis}

Quantification and the statistical analysis of the data were executed as previously described $^{47}$. Briefly, normality and variance of every dataset was tested. A paired or unpaired Student's t-test was used to probe the significance but for not normally distributed data, a nonparametric Mann-Whitney U-test and a Wilcoxon signed-rank test for paired analyses was used, respectively. In case of different variances, significance was probed with Welch's t-test and for not normally distributed data with Mood's median test. All data are presented as mean \pm 
s.e.m..Number of biological replicates (n) are illustrated in the respective figure legends or graphs. Significances are indicated with $*, \mathrm{P}<0.05 ; * *, \mathrm{P}<0.01$; ***, $\mathrm{P}<0.001$ in the Figures.

\section{Figure preparation}

All figures depicting structures were prepared using PyMOL (version 1.7.7.1) ${ }^{48}$. Electrostatic surface representations were generated using PDB2PQR (version 2.1.1) ${ }^{49}$ and APBS (version 1.3) ${ }^{50}$. Simulated annealing composite omit maps omitting waters in Extended data Fig. 2d were calculated in phenix.composite_omit_map (version 1.17) ${ }^{45}$ and sharpened in phenix.auto_sharpen (version 1.17) ${ }^{45}$ with an applied B-factor sharpening of $40.23 \AA$. Bar graphs and plots were made in GraphPad Prism (version 8.3.1) (GraphPad Software, LLC) and traces were made in OriginPro (version 9.1.0) (OriginLab Corporation). Chemical structures were drawn in ChemDraw (version 19.0.1.20) (PerkinElmer Informatics, Inc.). Sequence alignments and the phylogenetic tree were made using Clustal Omega ${ }^{51}$, Jalview (version 2.11.0) $)^{52}$ and Aline (version 1.0.025) ${ }^{53}$

\section{Synthesis of TASK-1 inhibitors BAY1000493 and BAY2341237}

The synthesis of BAY1000493 and BAY2341237 are described in patent number WO2017097792A1 and in the supplementary information file.

\section{Methods Only References}

36 Sitsel, O. et al. Crystallization of P-type ATPases by the High Lipid-Detergent (HiLiDe) Method. Methods Mol Biol 1377, 413-420 (2016).

37 Kabsch, W. Xds. Acta Crystallogr. D 66, 125-132 (2010).

38 Evans, P. Scaling and assessment of data quality. Acta Crystallogr. D 62, 72-82 (2006).

39 Winn, M. D. et al. Overview of the CCP4 suite and current developments. Acta Crystallogr D Biol Crystallogr 67, 235-242 (2011). 
40 Tickle, I. J. et al. STARANISO (http://staraniso.globalphasing.org/cgibin/staraniso.cgi). Cambridge, United Kingdom: Global Phasing Ltd. (2018).

41 McCoy, A. J. et al. Phaser crystallographic software. J Appl Crystallogr 40, 658-674 (2007).

42 Adams, P. D. et al. PHENIX: a comprehensive Python-based system for macromolecular structure solution. Acta Crystallogr D Biol Crystallogr 66, 213-221 (2010).

43 Emsley, P. et al. Features and development of Coot. Acta Crystallogr. D 66, 486-501 (2010).

44 BUSTER version 2.10.0 (Global Phasing Ltd, Cambridge, UK, 2011).

45 Liebschner, D. et al. Macromolecular structure determination using X-rays, neutrons and electrons: recent developments in Phenix. Acta crystallographica. Section D, Structural biology 75, 861-877 (2019).

46 Zuzarte, M. et al. Intracellular traffic of the K+ channels TASK-1 and TASK-3: role of N- and C-terminal sorting signals and interaction with 14-3-3 proteins. J Physiol 587, 929-952 (2009).

47 Rinne, S. et al. The molecular basis for an allosteric inhibition of $\mathrm{K}(+)$-flux gating in K2P channels. Elife 8 (2019).

48 Schrodinger, LLC. The PyMOL Molecular Graphics System, Version 1.7.7.1 (2010).

49 Dolinsky, T. J. et al. PDB2PQR: an automated pipeline for the setup of PoissonBoltzmann electrostatics calculations. Nucleic Acids Res 32, W665-667 (2004).

50 Jurrus, E. et al. Improvements to the APBS biomolecular solvation software suite. Protein Sci 27, 112-128 (2018).

51 Sievers, F. et al. Fast, scalable generation of high-quality protein multiple sequence alignments using Clustal Omega. Mol Syst Biol 7, 539 (2011). 
52 Waterhouse, A. M. et al. Jalview Version 2--a multiple sequence alignment editor and analysis workbench. Bioinformatics 25, 1189-1191 (2009).

53 Bond, C. S. \& Schuttelkopf, A. W. ALINE: a WYSIWYG protein-sequence alignment editor for publication-quality alignments. Acta Crystallogr D Biol Crystallogr 65, 510$512(2009)$.

\section{Author Information / Endnote}

Reprints and permissions information are available at www.nature.com/reprints.

M.D. M.H., H.M., T.M., are inventors on patent no. WO2017097792A1, Priority date 10 December, 2018, entitled "2-phenyl-3-(piperazinomethyl)imidazo[1,2-a]pyridine derivatives as blockers of TASK-1 and TASK-3 channels, for the treatment of sleep-related breathing disorders". N.D. is an inventor on patent no. EP18189182.1, Priority date 15 August, 2018, entitled "TASK-1 inhibitors for treatment of atrial arrhythmias". The other authors declare no competing interests. They were not involved in the development of the compounds described in this patent and will not benefit from the patent.

Correspondence and requests for materials should be addressed to N.D. (decher@staff.uni-

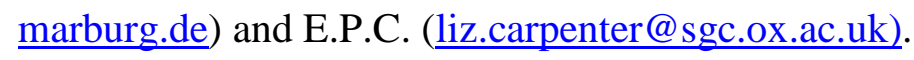

\section{Data Availability}

X-ray crystallography structures and datasets generated during the current study are deposited in the Protein Data Bank (PDB) with the following accession codes 6RV2 for the TASK-1, 6RV3 for the TASK-1/BAY1000493 complex and 6RV4 for the TASK-1/BAY2341237 structure. The source data for the electrophysiology measurements generated during the current 
study are available in the publication in Source Data Files associated with Figs. 2, 3 and 4 and Extended Data Figs. 1, 4 and 5. 


\section{Extended Data Figures}

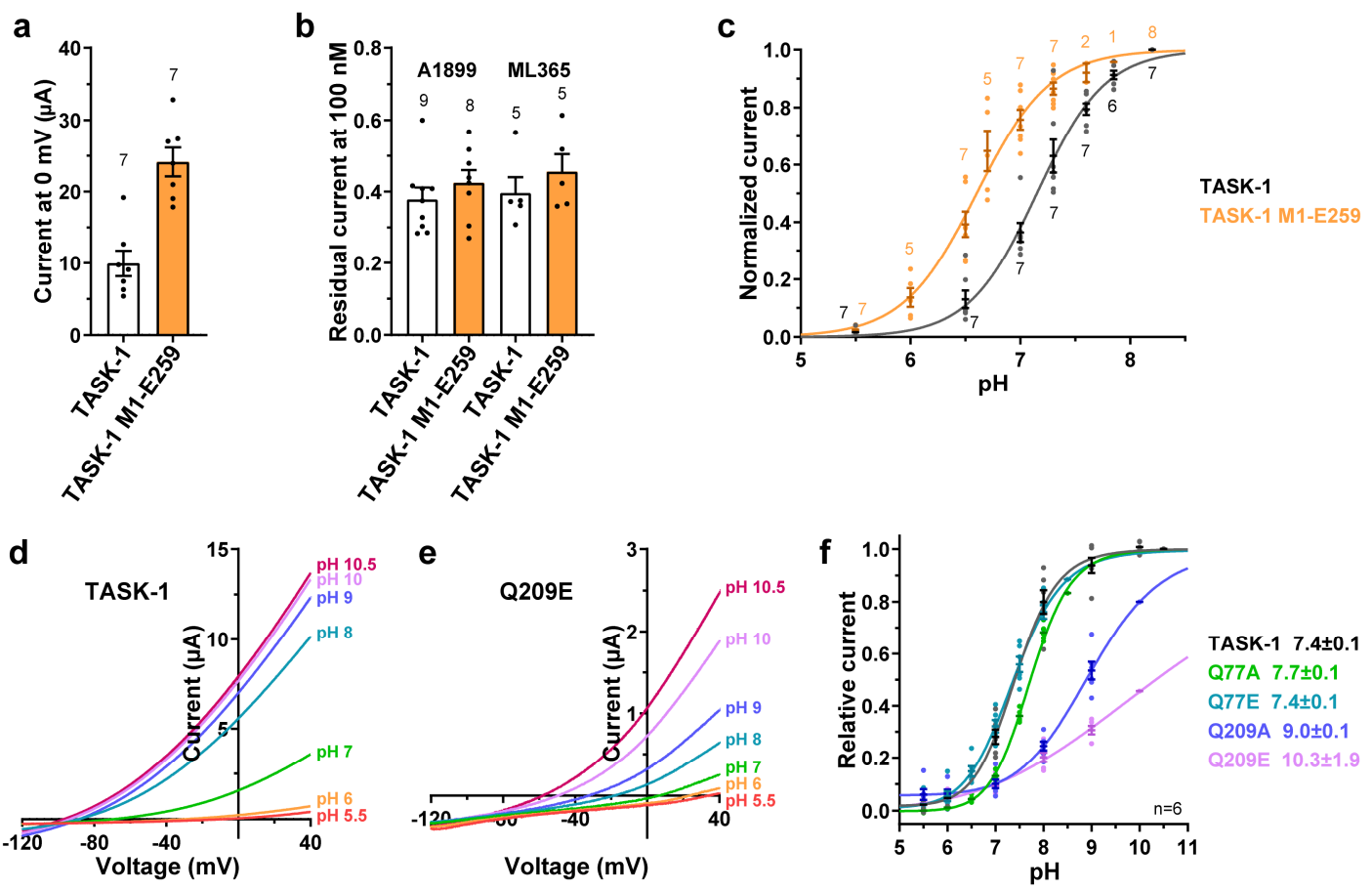

Extended Data Fig. 1 | Functional properties of the crystallisation construct TASK-1 ${ }^{\text {M1- }}$ E259 and pH-sensitivity of mutations near the pH sensor His98. a, The crystallisation construct TASK-1 ${ }^{\mathrm{M} 1-\mathrm{E} 259}$ has similar channel properties to WT protein. Whole-cell TASK-1 wild-type and TASK-1 ${ }^{\text {M1-E259 }}$ currents recorded after expression in Xenopus oocytes showing that the crystallisation construct is functionally active $(n=7)$. b, The truncated crystallisation construct TASK-1 ${ }^{\mathrm{M} 1-\mathrm{E} 259}$ retains normal inhibition by the pore-blockers A1899 (WT: $\mathrm{n}=9$, TASK-1 $\left.{ }^{\mathrm{M} 1-\mathrm{E} 259}: \mathrm{n}=8\right)$ and ML365 $(\mathrm{n}=5)$. c, The crystallisation construct retains sensitivity to changes in extracellular $\mathrm{pH}$ (number of biological repeats shown on in the figure). The small change in half maximal activation from $\mathrm{pH} 7.2$ to $\mathrm{pH} 6.4$ suggests the truncated cytoplasmic region may influence this process. But overall, the truncated construct exhibits a similar pharmacology and regulation to the wild-type channel. d, e, Representative two-electrode voltage-clamp recordings of wild-type TASK-1 $(n=6)$ and the Q209E $(n=6)$ mutant under different extracellular $\mathrm{H}^{+}$concentrations. $\mathbf{f}$, $\mathrm{pH}$ dose-response curves of wild-type TASK-1 $(\mathrm{n}=6)$ and mutants at Q77 $(\mathrm{n}=6)$ and Q209 $(\mathrm{n}=6)$, residues positioned above the $\mathrm{pH}$ sensor H98 $(n=6)$. Number of biological replicates (n) are illustrated in the legends or graphs. Data are presented as mean \pm s.e.m. and shown as individual points. 
a

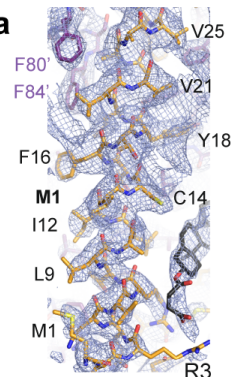

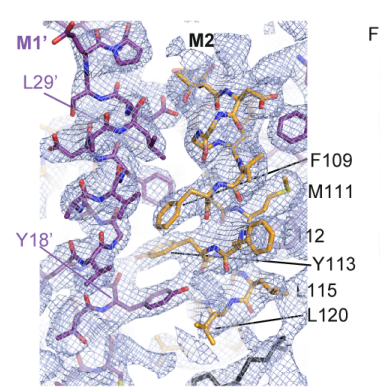
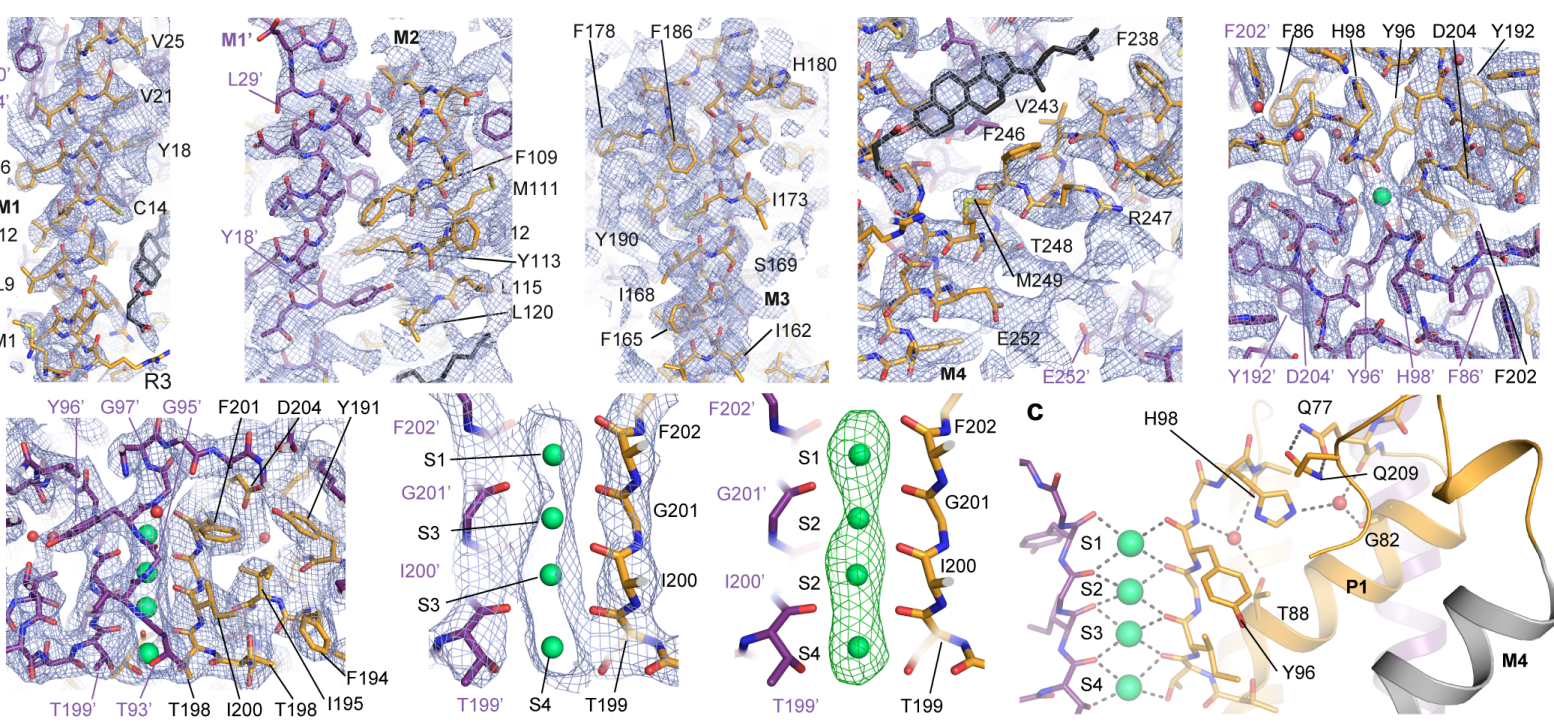

Y192' D204' Y96' H98' F86' F202

d

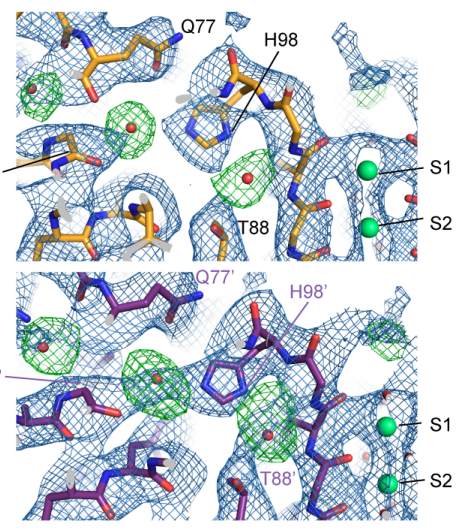

f

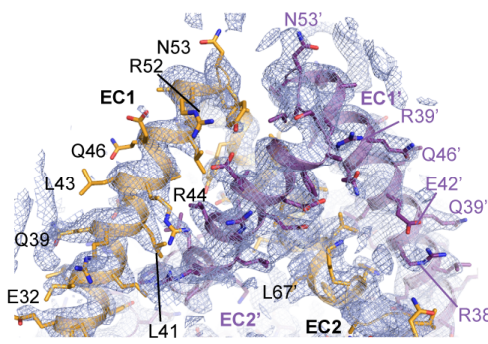

j

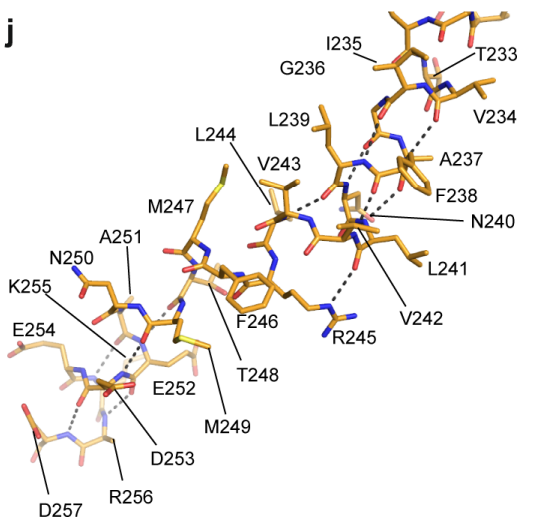

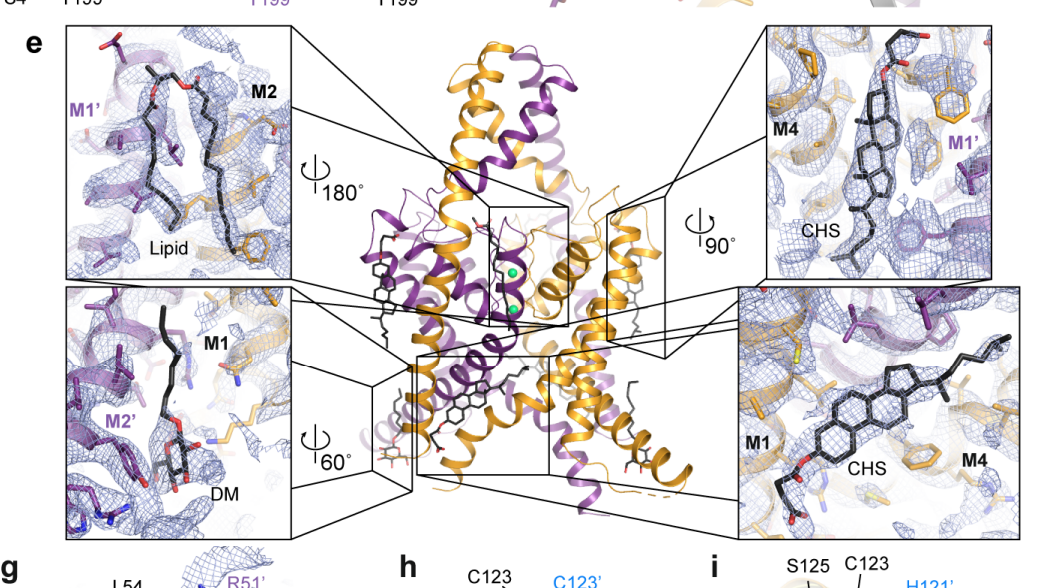

\section{g}

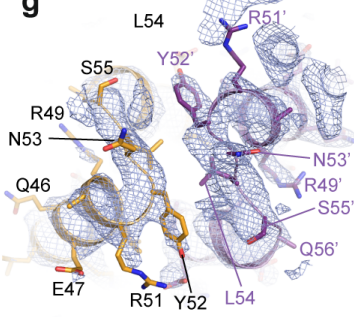

h
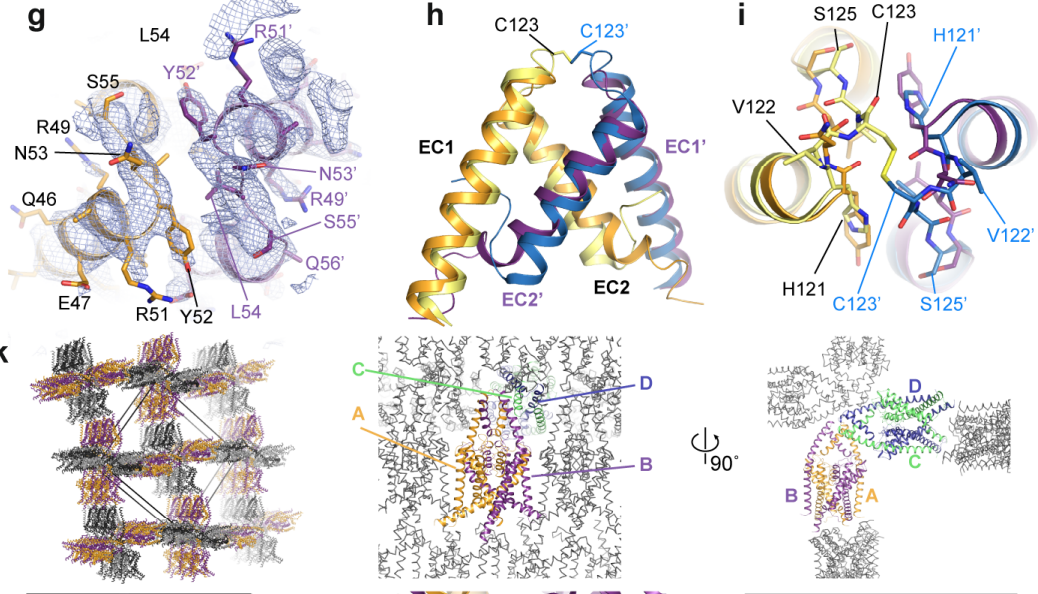

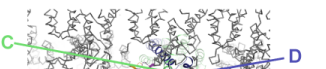
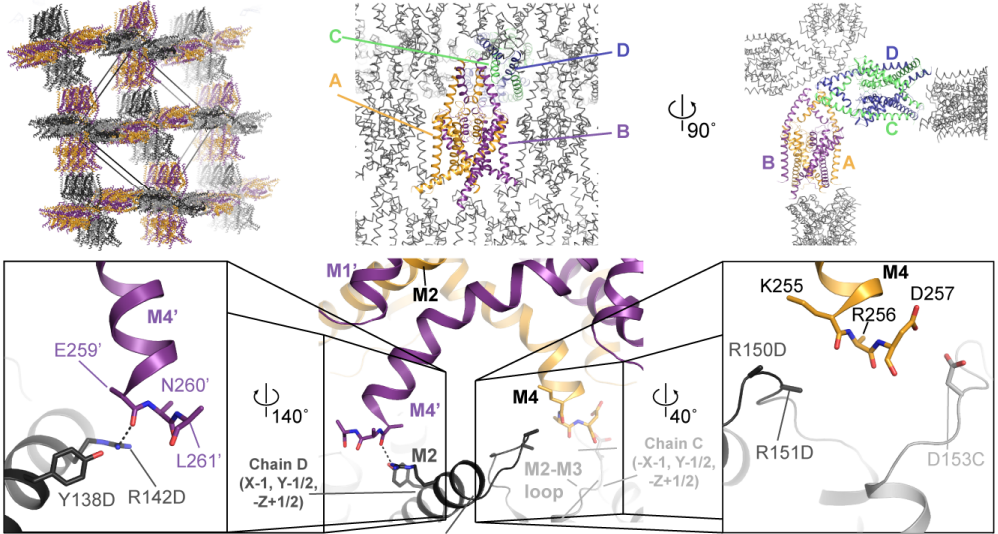
Extended Data Fig. 2 | The structure of TASK-1 at 3.0 $\AA$ resolution indicates that the cap in TASK-1 forms the classic cap domain structure without an intramolecular disulphide bond. a, Final BUSTER 2Fo-Fc electron density maps contoured at 1.0 $\sigma$, showing the M1-M4 helices and the selectivity filter from the plane above the membrane. b, The selectivity filter with a $2 \mathrm{Fo}-\mathrm{Fc}$ map contoured at $1.0 \sigma$ shown from the plane of the membrane (left), the selectivity filter with a $2 \mathrm{Fo}-\mathrm{Fc}$ map contoured at $2.0 \sigma$ (middle) and a simulated annealing mFo$\mathrm{DFc} \mathrm{K}^{+}$omit map contoured at $4.0 \sigma$ (right). c, The $\mathrm{pH}$ sensor residue, His98, the surrounding residues and the pore, with the $\mathrm{K}^{+}$ions shown in green. $\mathbf{d}$, Simulated annealing $2 \mathrm{mFo}-\mathrm{DFc}$ (blue) and mFo-DFc (green) water omit maps shown for waters around H98 (top) and H98' (bottom). Maps are contoured at $0.8 \sigma$ and $3.0 \sigma$ respectively and the $2 \mathrm{mFo}-\mathrm{DFc}$ map was sharpened in phenix.auto_sharpen with an applied B-factor sharpening of $40.23 \AA^{2}$. e, Cartoon representation of the TASK-1 structure, with lipids, detergents and CHS shown as sticks, with zoomed views of the binding sites. 2Fo-Fc maps are contoured at $1.0 \sigma$. f, 2Fo-Fc electron density maps contoured at $1.0 \sigma$ of the cap and $\mathbf{g}$, view of the cap apex. $\mathbf{h}$, superposition of the TASK-1 cap (gold and purple) and TREK-2 (PDB: 4XDJ) cap (yellow and blue), illustrating lack of disulphide bond in TASK-1 and $\mathbf{i}$, view of the aligned cap apices. $\mathbf{j}$, The M4 helix residues 233-257 represented as sticks, with the main chain hydrogen bonding pattern shown as dashed lines. k, Diagrams of the crystal packing showing overall packing interactions (top left), the crystal packing around the $\mathrm{AB}$ (gold and purple) and $\mathrm{CD}$ (blue and green) dimers (top middle and right) and a close-up of the X-gate and distal M4 crystal contacts (bottom). 
a

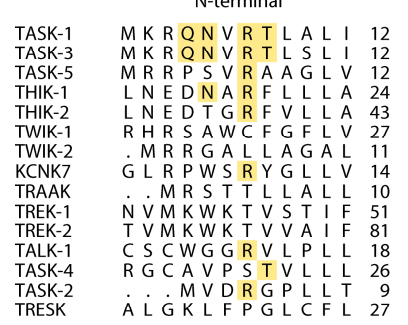

Cap apex

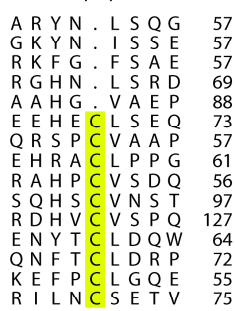

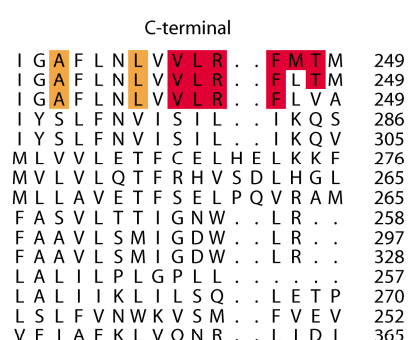

\section{$\square$ X-gate}

$\triangle$ Hinge

$\bigcirc$ Latch

$\nabla$ Selectivity filter

$\hat{z} \mathrm{pH}$ sensor

¿ 2 PPH4 mutation

Birk-Barel syndrome mutation

$\diamond$ Compound binding site b

TASK-1 H. sapiens
TASK-1 M. musculus
TASK-1 G. gallus
TASK-1 D. rerio
TASK-3 H. sapiens
TASK-3 H. vulgaris
TASK-3 S. purpuratus
TASK-3 B. belcheri
TASK-3 O. bimaculoides
TASK-3 C. elegans
TASK-5 H. sapiens

TASK-3 C. elegans

TASK-7 D. melanogaster

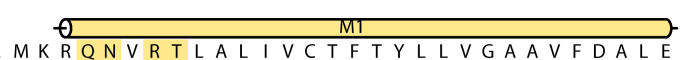
MKRQNVRTLALIVCTFTYLLVGAAVFDALESEPEMIERQR 40 MKRQNVRTLALIMCTFTYLLVGAAVFDALESEEETAERRR MKRQN I RTLVLI I C TFTYLLVGAAVFDALESKME I TQKK I

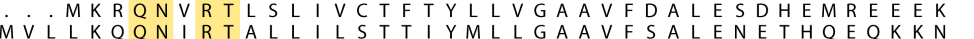
$M F K V L I M K R Q N V R T L S L I V C T F \mid Y L L L G A A V F D A L E S R N E E R E K N R$ MHFLLLLAMKKQN I RTLSLMIASFMYLLVGAAVFDALESESEEKQRRL MKKONVRTLSLIVCTFTYLLVGAAVFDALESEYENEMKRK 40

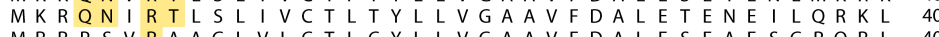
MRRPSVRAAGLVLCTLCYLLVGAAVFDALESEAESGRQRL 40 MMKRQNVRTLSLVVCTFTYLLIGAAVFDSLESPTEAKRWEF OO OO

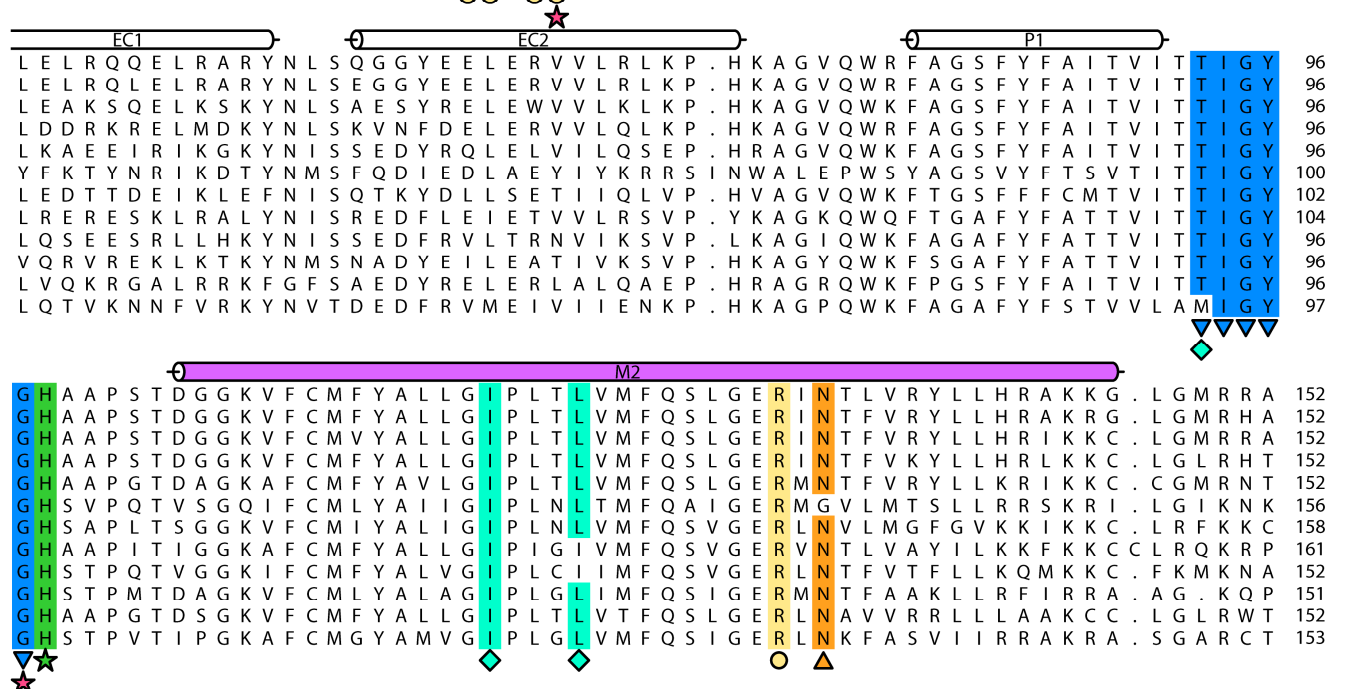

C
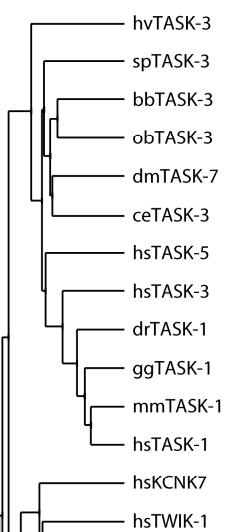

- hsTWIK-1 - hsTWIK-2
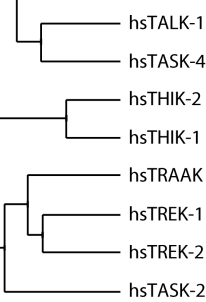

hsTASK-2
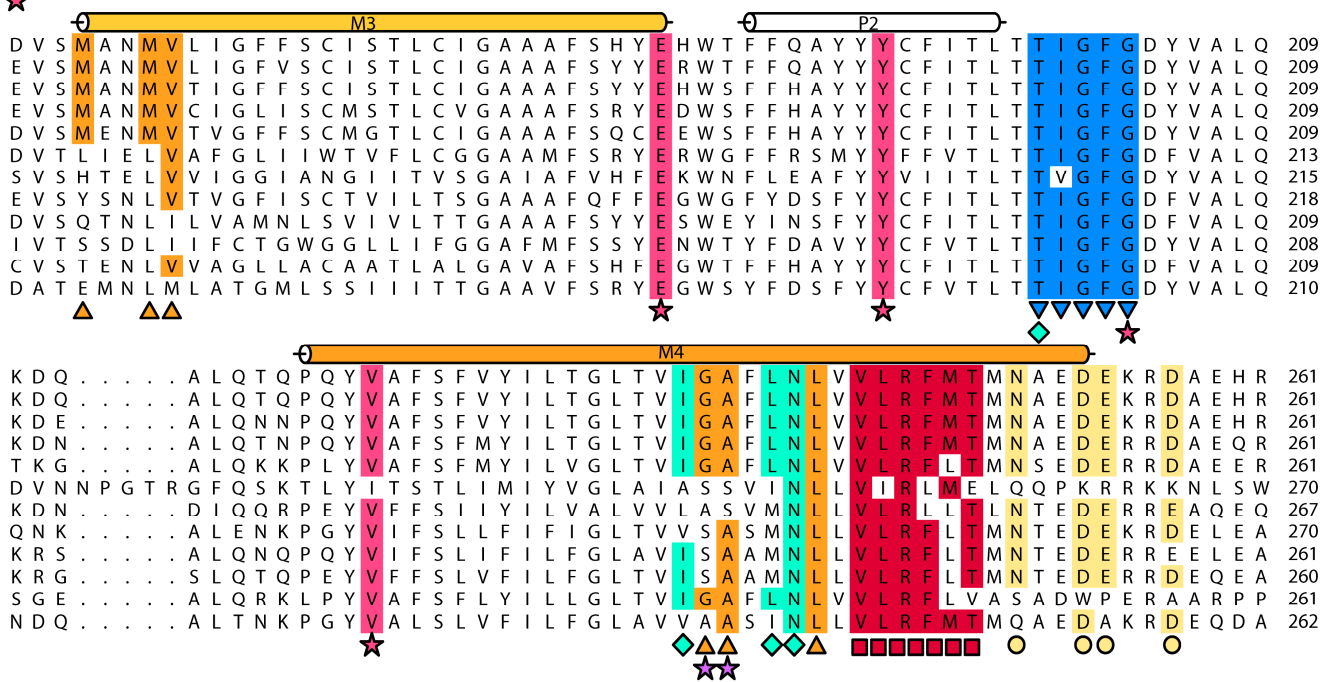

Extended Data Fig. 3 | Alignment and phylogenetic trees for the TASKs and $K_{2 P}$ channels reveal conservation of $\mathrm{X}$-gate residues in TASK-1, 3 and 5, and a lack of an X-gate in other $\mathrm{K}_{2 \mathrm{P}}$ channels. , Alignment of the human $\mathrm{K}_{2 \mathrm{P}}$ proximal M1, cap and distal M4 regions. The cysteine residue in the cap apex is highlighted in yellow and the X-gate residues in red. $\mathbf{b}$, 
Sequence alignment of TASK channels from different species. c, Phylogenetic tree of the human $\mathrm{K}_{2 \mathrm{PS}}$ and the TASK channels in $\mathbf{b}$. The TASK-1, -3 and -5 channels are highly conserved, whereas the $\mathrm{K}_{2 \mathrm{P}}$ channels that were originally designated at TASK-2 and TASK4/TALK-2 have much lower homology and belong in the subfamily of TALK channels. 

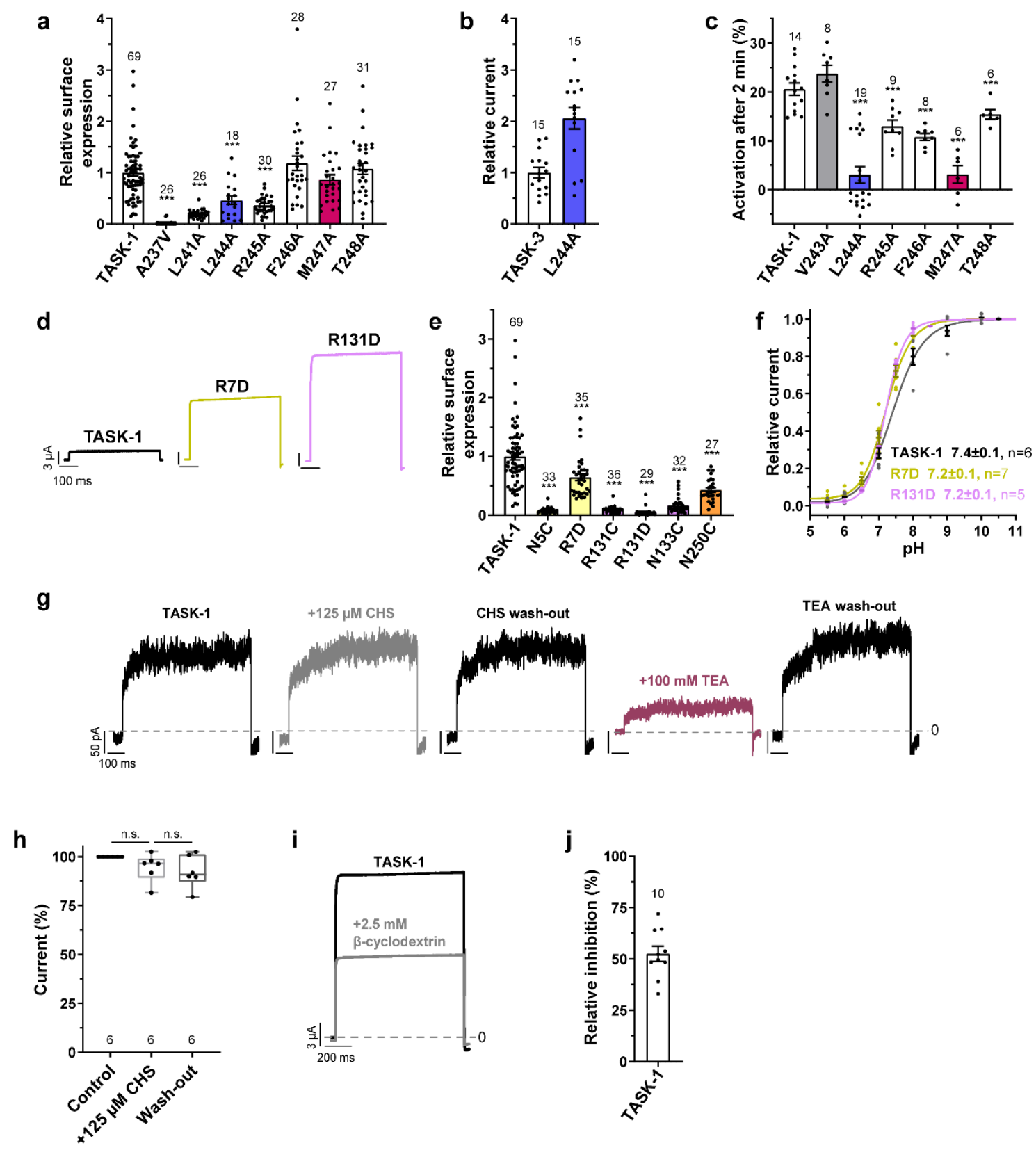

Extended Data Fig. 4 | Functional studies of TASK X-gate and latch mutants and role of cholesterol on channel function. a, Relative surface expression of wild-type TASK-1 and gain-of-function mutations at the bend or the X-gate (two-sided Mood's median test, $P_{\mathrm{A} 237 \mathrm{~V}}=3.2 \mathrm{E}-09, \quad P_{\mathrm{L} 241 \mathrm{~A}}=3.2 \mathrm{E}-09, \quad P_{\mathrm{R} 245 \mathrm{~A}}=1.4 \mathrm{E}-09 ; \quad$ two-sided Mann-Whitney-U-test $\left.P_{\mathrm{L} 244 \mathrm{~A}}=3.0 \mathrm{E}-05\right)$. b, Relative current amplitudes of wild-type TASK-3 $(\mathrm{n}=15)$ and Leu244Ala (n=15) (two-sided Welch's t-test, $P=1.8 \mathrm{E}-04)$. c, Analysis of $1 \mathrm{mM}$ sevoflurane application to wild-type and mutated TASK-1 (two-sided Student's t-test, $P_{\mathrm{V} 243 \mathrm{~A}}=1.5 \mathrm{E}-01, P_{\mathrm{R} 245 \mathrm{~A}}=5.3 \mathrm{E}-04$, $P_{\mathrm{M} 247 \mathrm{~A}}=3.8 \mathrm{E}-07$; two-sided Mann-Whitney-U-test $P_{\mathrm{L} 244 \mathrm{~A}}=2.4 \mathrm{E}-06$; two-sided Welch's t-test $\left.P_{\mathrm{F} 246 \mathrm{~A}}=1.6 \mathrm{E}-06, P_{\mathrm{T} 248 \mathrm{~A}}=4.3 \mathrm{E}-03\right)$. d, Representative two-electrode voltage-clamp recordings of TASK-1 wild-type ( $n=32)$ and the latch destabilizing mutants $\operatorname{Arg} 7 \operatorname{Asp}(n=48)$ and $\operatorname{Arg} 131 \operatorname{Asp}$ 
$(\mathrm{n}=15)$. e, Relative surface expression of wild-type TASK-1 and TASK-1 mutations in the latch region (two-sided Mood's median test, $P_{\mathrm{N} 5 \mathrm{C}}=2.9 \mathrm{E}-12, \quad P_{\mathrm{R} 7 \mathrm{D}}=1.3 \mathrm{E}-05, \quad P_{\mathrm{R} 131 \mathrm{C}}=2.3 \mathrm{E}-13$, $\left.P_{\mathrm{R} 131 \mathrm{D}}=1.4 \mathrm{E}-09, P_{\mathrm{N} 133 \mathrm{C}}=1.2 \mathrm{E}-11, P_{\mathrm{N} 250 \mathrm{C}}=1.8 \mathrm{E}-07\right)$. f, Normal extracellular $\mathrm{pH}$ gating wild-type TASK-1 $(n=6)$ and of mutant channels with a destabilization of the inner gate, by latch mutants R7D ( $n=7)$ and R131D ( $n=5)$. g, Representative inside-out macropatch recordings of TASK-1 wild-type channels after consecutive application of CHS, control, TEA and again control solution ( $\mathrm{n}=6)$. $\mathbf{h}$, Analysis of CHS applied to inside-out patch clamp recordings via a rapid perfusion system (two-sided paired Student's t-test, $P_{\text {control-CHS }}=1.2 \mathrm{E}-01, P_{\text {CHS-wash-out }}=7.6 \mathrm{E}-02$ ). i, Representative two-electrode voltage-clamp recordings before and after 30 minutes of $\beta$-cyclodextrin application $(\mathrm{n}=10)$. $\mathbf{j}$, Analysis of relative inhibition by $2.5 \mathrm{mM} \beta$-cyclodextrin. All data were recorded in X. laevis oocytes. Number of biological replicates (n) are illustrated in the respective figure legends or graphs. n.s., not significant; ***, $P<0.001$. Data in a,b,c,e,g,j are presented as mean \pm s.e.m.. Box plots in h, the centre line represents the median, the box limits are the 25th and 75th percentiles, and the whiskers are s.e.m.. For full statistical details see Methods and Source Data File. 
BAY1000493

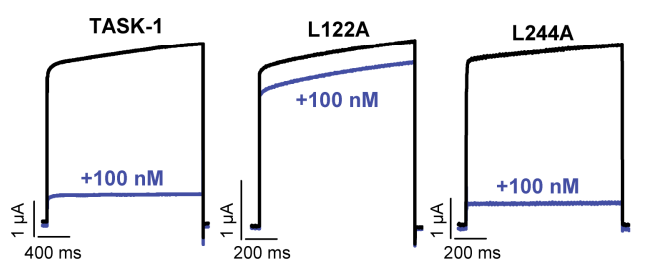

C
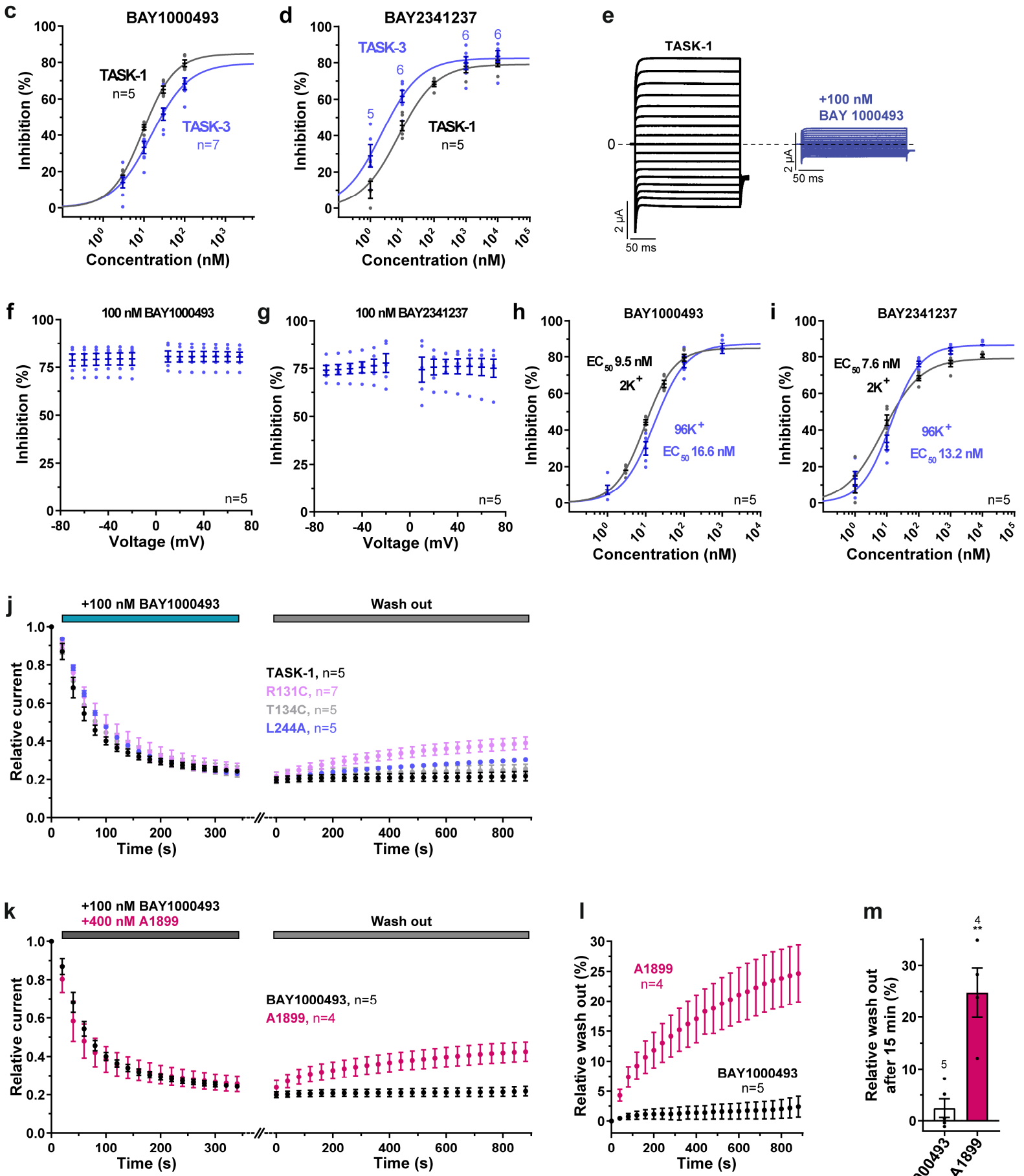

BAY1000493
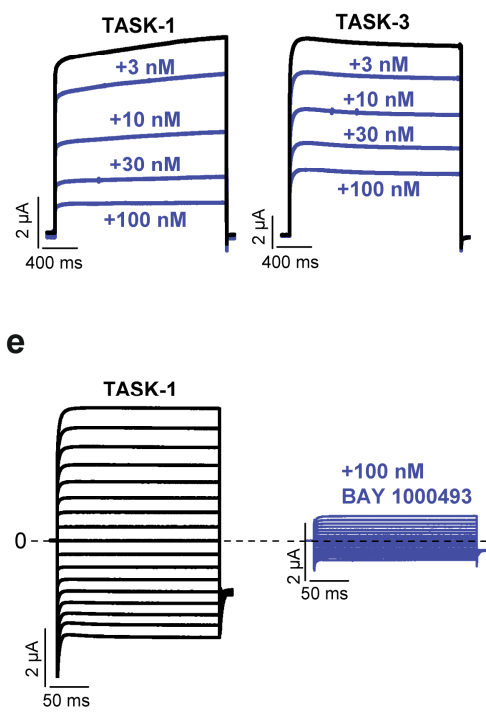

Concentration (nM)

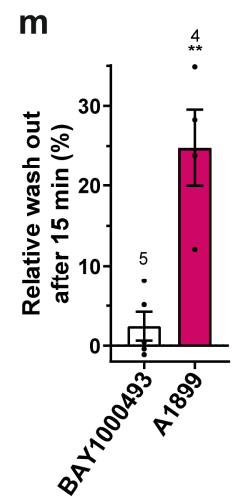




\section{Extended Data Fig. 5 | Effects of BAY1000493 and BAY2341237 on TASK-1 and TASK-}

3, and inhibitor washout data for BAY1000493 and A1899 inhibitor. a, Representative twoelectrode voltage-clamp recordings of TASK-1 wild-type $(n=5)$, Leu122Ala $(n=5)$ and Leu244Ala (n=4) mutants before and after application of BAY1000493 (100 nM). b, Representative recordings of TASK-1 $(n=5)$ and TASK-3 ( $n=7)$ wild-type channels before and after application of different concentrations of BAY1000493. c, Dose response curves for BAY1000493 on TASK-1 (n=5) and TASK-3 (n=7). d, Dose response curves for BAY2341237 on TASK-1 ( $n=5)$ and TASK-3 ( $n=6$; except for $1 \mathrm{nM}, \mathrm{n}=5)$. e, Representative recordings of TASK-1 wild-type before ( $n=5)$ and after $(n=5)$ application of BAY1000493 (100 nM) under high extracellular potassium concentration (symmetrical conditions), using an ND96 solution in which $96 \mathrm{mM} \mathrm{NaCl}$ was replaced by $\mathrm{KCl}$. A step protocol with an increment of $+10 \mathrm{mV}$ was applied every 12 s. f, g, Analyses of the voltage-dependence of TASK-1 inhibition by BAY1000493 and BAY2341237. h, i, The EC50 values were determined in an ND96 solution containing $2 \mathrm{mM}$ extracellular potassium or in ND96 solution with $96 \mathrm{mM} \mathrm{NaCl}$ replaced by KCl. j, Washout of BAY1000493 from TASK-1 ( $n=5)$ and TASK-1 mutants Arg131Cys (n=7), Thr134Cys ( $\mathrm{n}=5)$, Leu244Ala ( $\mathrm{n}=5)$. k, Washout of BAY1000493 ( $\mathrm{n}=5)$ compared to A1899 $(n=4)$. l, relative washout rates for BAY1000493 (n=5) and A1899 $(n=4)$. m, Analysis of the washout rates for the compounds shown in $\mathbf{l}$ (using two-sided Student's t-test, $P_{\mathrm{A} 1899}=2.0 \mathrm{E}-03$ ). Number of biological replicates (n) are illustrated in the respective figure legends or graphs. **, $P<0.01$. Data are presented as mean \pm s.e.m..For full statistical details see Methods and Source Data File. 

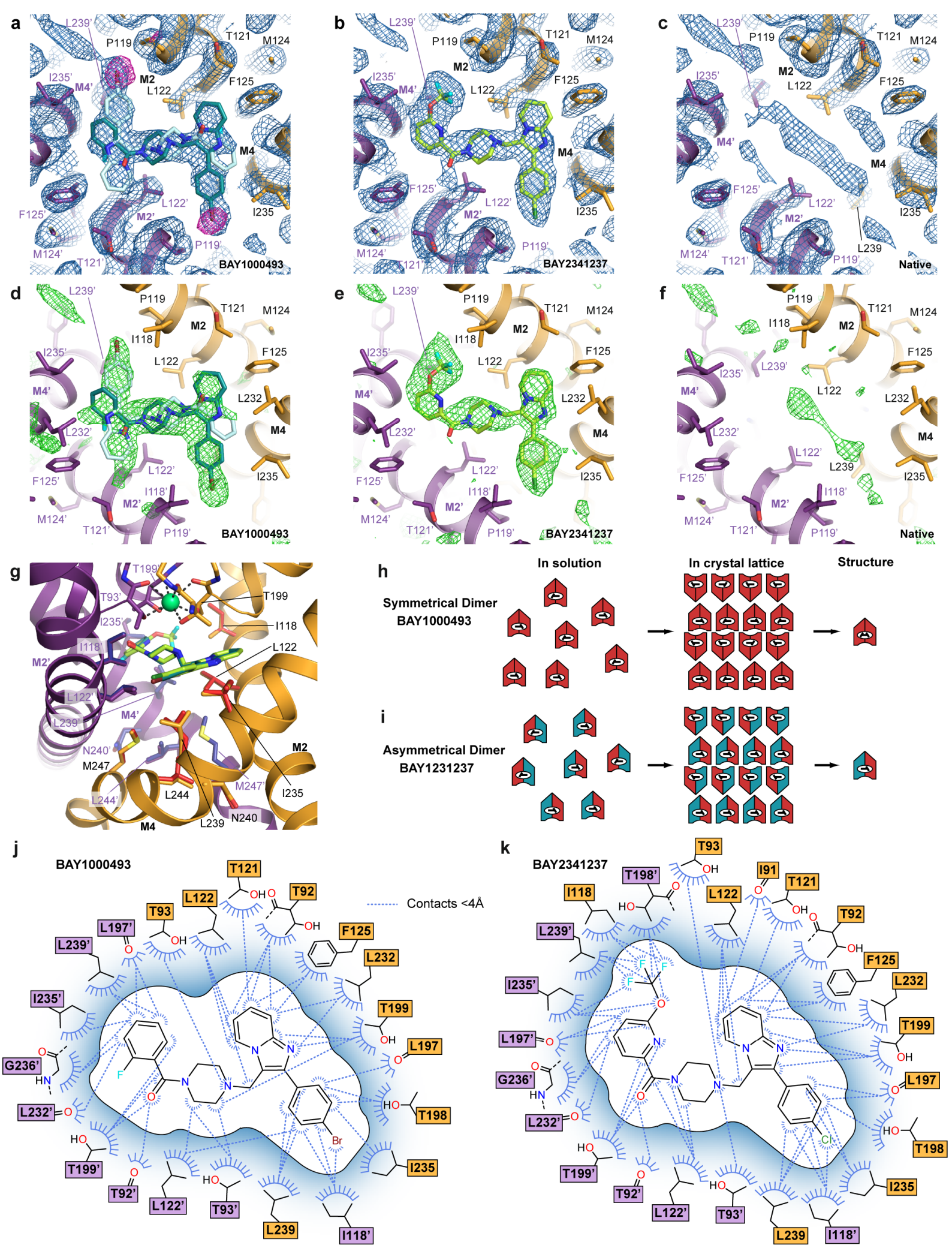

Extended Data Fig. 6 | Structures, electron density maps and bromine anomalous data for TASK-1 in complex with BAY1000493 or BAY2341237. a, The TASK-1/BAY1000493 complex structure, viewed from the selectivity filter, towards the vestibule, with the $2 \mathrm{Fo}-\mathrm{Fc}$ map (blue) and the anomalous difference map at $3.3 \AA$ collected at the $\mathrm{Br}$ edge (magenta). The 
two 50\% occupancy BAY1000493 molecules shown with carbons coloured teal or light blue, red oxygens, dark blue nitrogens, cyan fluorines and maroon bromines. b, The TASK1/BAY2341237 complex structure, as for a, with the 2Fo-Fc map (blue). 100\% occupancy BAY1000493 molecule shown with green carbons, dark green chlorines and other atoms as in a. c, The TASK-1 structure, with an $2 \mathrm{Fo}-\mathrm{Fc}$ map (blue). There is some residual density below the selectivity filter, as is seen in many $\mathrm{K}_{2 \mathrm{P}}$ structures. $\mathbf{d}$, e, and $\mathbf{f}$, represent the same structures as in $\mathbf{a}, \mathbf{b}$ and $\mathbf{c}$, with the positive Fo-Fc difference density from omit maps (green) calculated with the inhibitors excluded. 2Fo-Fc and Fo-Fc maps are contoured at $1.0 \sigma$ and $2.5 \sigma$ respectively. The anomalous difference map in $\mathbf{a}$ is contoured at $3.5 \sigma$. $\mathbf{g}$, Superposition of the two complexes seen from the membrane plane with BAY1000493 in teal and BAY2341237 in green. The BAY1000493 complex is shown as cartoon and side chains as sticks (gold and purple) and sidechains from the BAY2341237 complex in red and blue. h, schematic showing how 50:50 distribution of BAY1000493 occurs in crystals. i, schematic showing how asymmetry could lead to $100 \%$ occupancy in one orientation in the BAY2341237 case. $\mathbf{j}$, $\mathbf{k}$, interactions between TASK-1 and BAY1000493 or BAY2341237. Close contacts $(<4.0 \AA)$ between the protein and inhibitor atoms are shown as blue lines. For clarity, only the three closest contacts are shown if there were more than three contacts. 

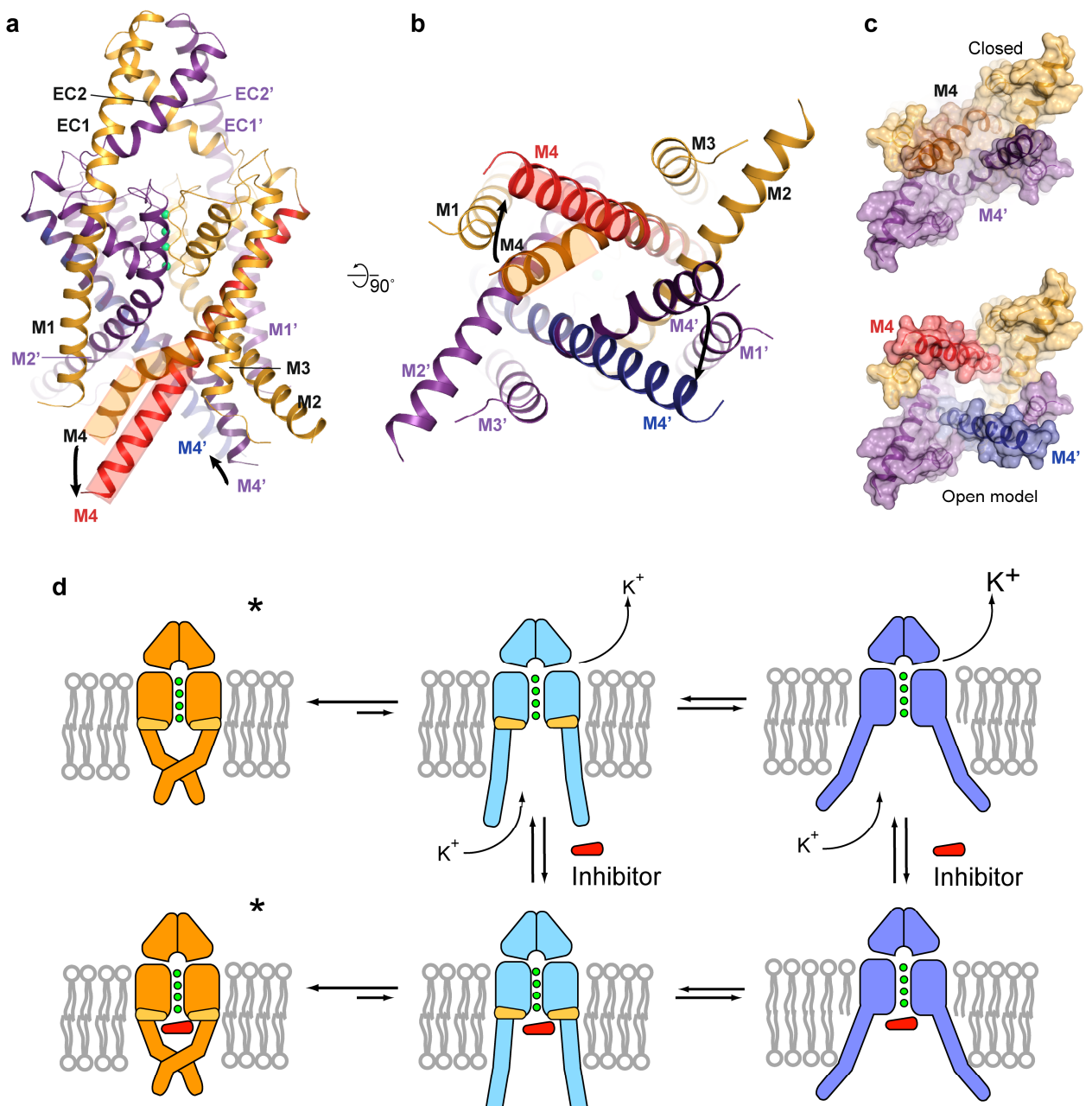

Closed
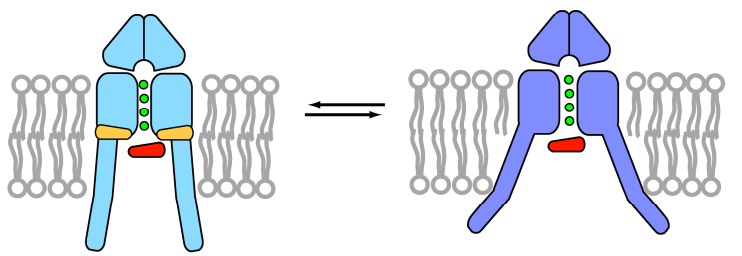

$$
\begin{gathered}
\text { Open X-gate } \\
\text { Helices in "down" } \\
\text { conformation } \\
\text { Fenestrations open } \\
\text { Lower activity state }
\end{gathered}
$$

Open channel Helices in "up" conformation Fenestrations closed Higher activity state

\section{Extended Data Fig. 7 | Model of the conformation that TASK-1 could adopt with a} straight, continuous M4 helix and a gating scheme for TASK-1. a,b Model of TASK-1 with M4 adopting a straight $\alpha$-helical conformation (in red and blue) aligned with the TASK-1 structure shown from $\mathbf{a}$, the side and $\mathbf{b}$, below the membrane. $\mathbf{c}$, The TASK-1 structure and open model viewed from below the membrane. $\mathbf{d}$, A schematic model for TASK-1 activation, with the closed X-gate state shown in orange, and two schematics for possible open states, based on the "down" and "up" states seen in TRAAK and TREK-2. The state based on the "down" state with an open X-gate, is shown in blue and the version with the helices in the "up" state, potentially giving a more active state is shown in mauve. Stars indicate the TASK-1 conformations for which we have structures. At acidic pHs, we predict that His98 would move, causing the selectivity filter to become less conductive, a change that could in theory occur in any of the conformations shown above. 


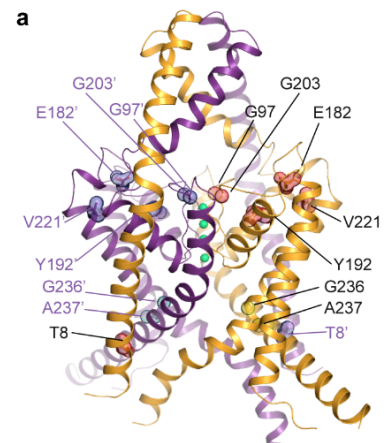

d
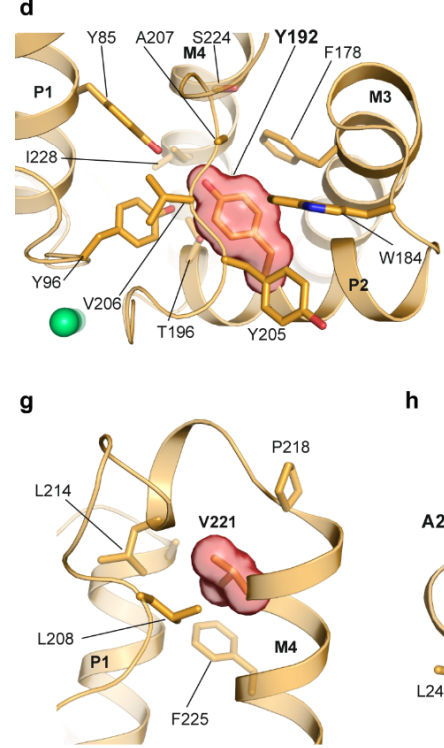

h b
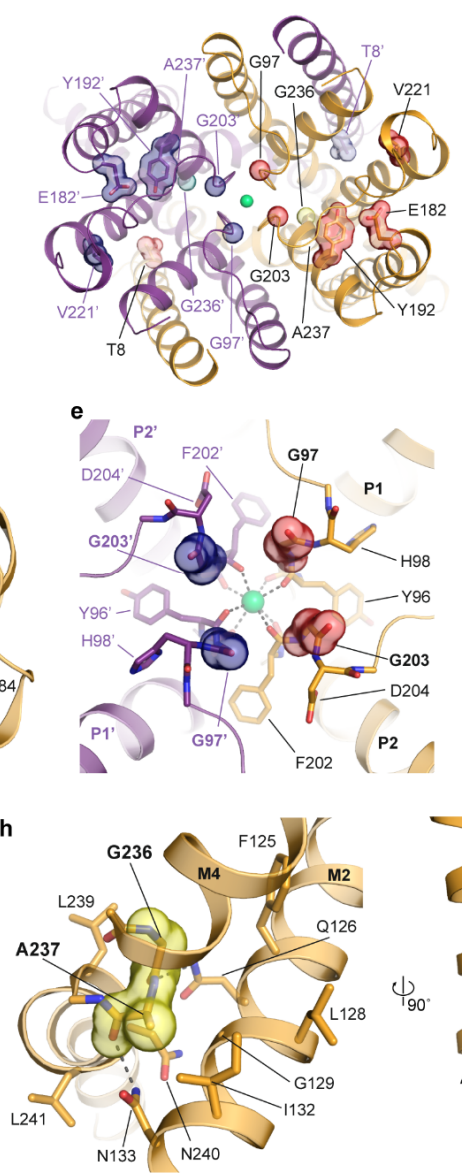
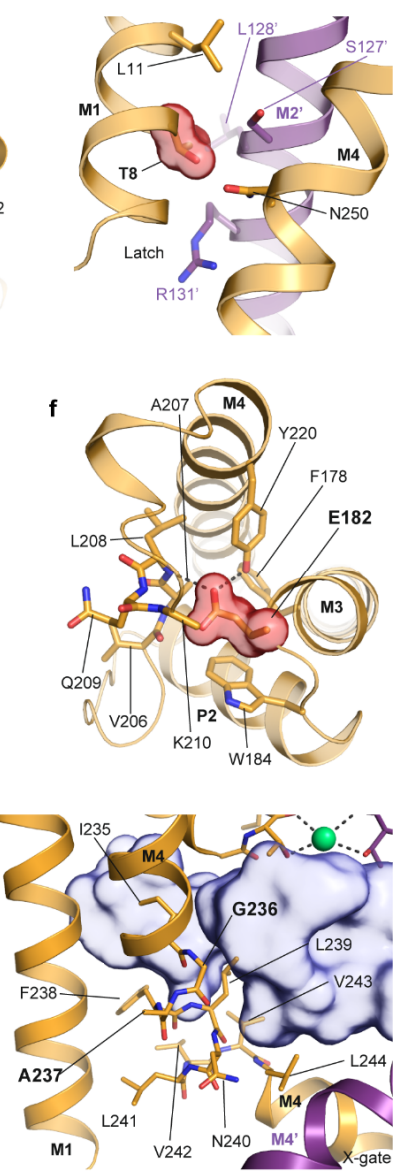

Extended Data Fig. 8 | TASK-1 mutations associated with PPH4 - structure and location of mutations. Location of mutations in TASK-1 and TASK-3 mapped onto the TASK-1 structure shown from $\mathbf{a}$, the membrane plane and $\mathbf{b}$, from the extracellular side. Close-up views of $\mathbf{c}$ Thr8Lys in the latch region on M1, d, Tyr192Cys, e, Gly97Arg and Gly203Asp in the pore loops, f, Glu182Lys, and g,Val221Leu . h, the TASK-3 Birk-Barel syndrome mutations Gly236Arg and Ala237Asp in M4 (highlighted in yellow in the panel to the left), shown from the membrane plane and rotated $90^{\circ}$ with the vestibule shown in blue in the panel to the right. 


\begin{tabular}{|c|c|c|c|c|c|c|c|c|}
\hline & \multicolumn{2}{|c|}{ TASK-1 (6RV2)a } & \multicolumn{2}{|c|}{ BAY1000493 complex (6RV3) } & \multicolumn{2}{|l|}{$\begin{array}{l}\text { BAY1000493 } \\
\text { Br edge data }\end{array}$} & \multicolumn{2}{|c|}{$\begin{array}{l}\text { BAY2341237 complex } \\
\text { (6RV4) }^{b}\end{array}$} \\
\hline Data collection & & & & & & & & \\
\hline $\begin{array}{l}\text { Space group } \\
\text { Cell dimensions }\end{array}$ & \multicolumn{2}{|l|}{$\mathrm{P} 22_{12}{ }_{1}$} & \multicolumn{2}{|l|}{$\mathrm{P} 22_{1} 2_{1}$} & \multicolumn{2}{|l|}{$\mathrm{P} 22_{12}{ }_{1}$} & \multicolumn{2}{|l|}{$\mathrm{P} 222_{1}$} \\
\hline $\begin{array}{l}a, b, c(\AA) \\
a, \beta, \gamma\left({ }^{\circ}\right)\end{array}$ & \multicolumn{2}{|c|}{$45.14,204.79,238.34$} & \multicolumn{2}{|c|}{$45.07,204.50,239.32$} & \multicolumn{2}{|c|}{$45.03,201.91,238.91$} & \multicolumn{2}{|c|}{$45.10,201.33,238.57$} \\
\hline Processing & anisotropic & isotropic & anisotropic & isotropic & anisotropic & isotropic & anisotropic & isotropic \\
\hline Resolution $(\AA)$ & $\begin{array}{l}47.67-3.00 \\
(3.12-3.00)^{\mathrm{c}}\end{array}$ & $\begin{array}{l}47.67-3.00 \\
(3.11-3.00)^{\mathrm{c}}\end{array}$ & $\begin{array}{l}44.29-2.90 \\
(3.01-2.90)^{c}\end{array}$ & $\begin{array}{l}44.29-2.90 \\
(3.00-2.90)^{c}\end{array}$ & $\begin{array}{l}44.67-3.30 \\
(3.37-3.30)^{c}\end{array}$ & $\begin{array}{l}44.67-3.30 \\
(3.46-3.30)\end{array}$ & $\begin{array}{l}43.12-3.10 \\
(3.23-3.10)^{c}\end{array}$ & $\begin{array}{l}44.01-3.10 \\
(3.23-3.10)^{c}\end{array}$ \\
\hline$R_{\text {merge }}$ & $0.215(1.021)$ & $0.255(1.946)$ & $0.168(0.745)$ & $0.224(1.710)$ & $0.492(3.007)$ & $0.516(2.743)$ & $0.187(0.606)$ & $0.235(1.299)$ \\
\hline$|/ \sigma|$ & $5.6(1.9)$ & $4.4(1.0)$ & $7.2(2.5)$ & $6.0(1.3)$ & $10.2(2.0)$ & $10.0(2.3)$ & $6.6(2.7)$ & $5.6(1.3)$ \\
\hline $\mathrm{CC}_{1 / 2}$ & $0.993(0.697)$ & $0.989(0.346)$ & $0.998(0.795)$ & $0.991(0.467)$ & $0.999(0.741)$ & $0.997(0.810)$ & $0.998(0.792)$ & $0.989(0.542)$ \\
\hline Completeness (\%) & $79.7(37.2)$ & $99.7(99.8)$ & $80.0(40.0)$ & $99.3(97.7)$ & $98.0(85.0)$ & $100(100)$ & $80.9(34.8)$ & $99.7(99.7)$ \\
\hline Redundancy & $5.3(5.7)$ & $5.1(5.3)$ & $3.9(3.8)$ & $3.9(3.9)$ & $34.3(34.5)$ & $34.3(35.2)$ & $3.5(3.7)$ & $3.5(3.6)$ \\
\hline No. reflections / & $193,749 /$ & $231,490 /$ & $157,963 /$ & $196,329 /$ & $1,145,529 /$ & $1,169,177 /$ & $117,488 /$ & $143,532 /$ \\
\hline $\begin{array}{l}\text { unique } \\
\text { Anomalous } \\
\text { completeness (\%) }\end{array}$ & 36,417 & 45,475 & 40,478 & 50,143 & $\begin{array}{l}33,435 \\
98.4(88.6)\end{array}$ & $\begin{array}{l}34,128 \\
99.9(99.9)\end{array}$ & 33,101 & 40,728 \\
\hline $\begin{array}{l}\text { Anomalous } \\
\text { redundancy }\end{array}$ & & & & & $18.5(17.8)$ & $17.3(17.5)$ & & \\
\hline \multicolumn{9}{|l|}{ Refinement } \\
\hline Resolution $(\AA)$ & \multicolumn{2}{|l|}{$47.0-3.0$} & \multicolumn{4}{|l|}{ 42.5- 2.9} & \multicolumn{2}{|l|}{$41.2-3.1$} \\
\hline No. reflections & \multicolumn{2}{|l|}{36,373} & \multirow{2}{*}{\multicolumn{2}{|c|}{$\begin{array}{l}40,419 \\
2354 / 2421\end{array}$}} & & & \multicolumn{2}{|l|}{33,045} \\
\hline$R_{\text {work }} / R_{\text {free }}(\%)$ & \multirow{2}{*}{\multicolumn{2}{|c|}{$25.42 / 25.70$}} & \multirow{2}{*}{\multicolumn{2}{|c|}{$23.54 / 24.21$}} & & & \multirow{2}{*}{\multicolumn{2}{|c|}{$25.08 / 25.60$}} \\
\hline No. atoms & & & & & & & & \\
\hline Protein & \multicolumn{2}{|l|}{8009} & \multicolumn{2}{|l|}{8070} & & & \multicolumn{2}{|l|}{7985} \\
\hline Ligand/ion & \multicolumn{2}{|l|}{426} & \multirow{2}{*}{\multicolumn{2}{|c|}{475}} & & & \multicolumn{2}{|l|}{405} \\
\hline Water & \multicolumn{2}{|l|}{31} & & & & & - & \\
\hline$B$-factors & & & & & & & & \\
\hline Protein & 66.13 & & 47.86 & & & & 48.58 & \\
\hline Ligand/ion & 80.93 & & 63.78 & & & & 53.41 & \\
\hline Water & 39.03 & & 27.92 & & & & - & \\
\hline R.m.s. deviations & & & & & & & & \\
\hline Bond lengths $(\AA)$ & 0.008 & & 0.008 & & & & 0.008 & \\
\hline Bond angles $\left({ }^{\circ}\right)$ & 0.904 & & 0.915 & & & & 0.896 & \\
\hline
\end{tabular}

\section{Extended Data Table 1 |X-ray crystallography data, refinement and model statistics.}

Footnotes for Extended Data Table 1:

${ }^{\text {a }}$ Data were collected from two crystals.

${ }^{\mathrm{b}}$ Data were collected from single crystals.

${ }^{\mathrm{c}}$ Values in parentheses are for highest-resolution shell. 


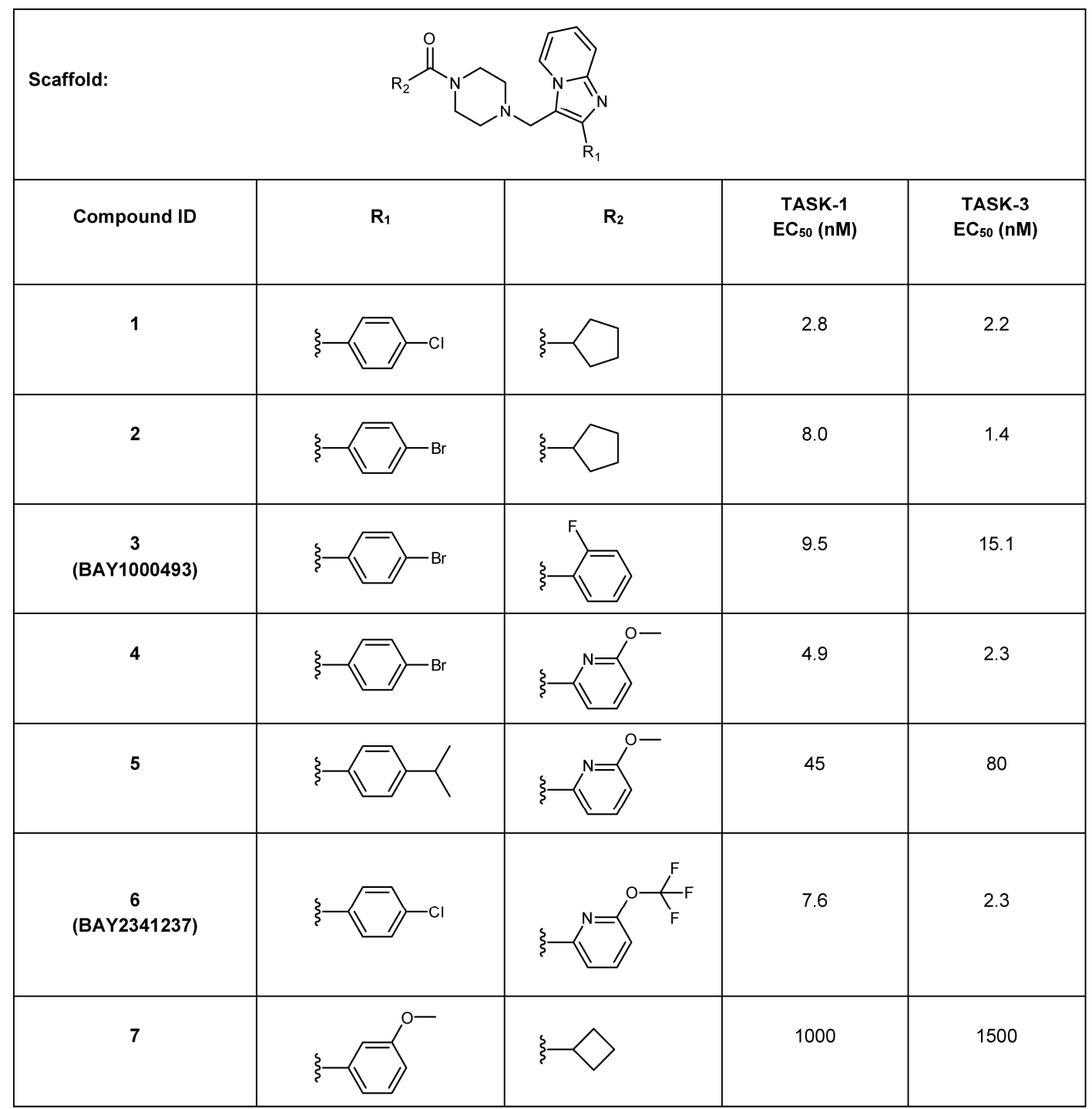

Extended Data Table $2 \mid$ EC 50 values of a series of TASK-1 compounds and their chemical structures. All EC50 values were measured with TEVC recordings in X. laevis oocytes. 


\title{
Supplementary information for:
}

\section{"A lower X-gate in TASK channels traps inhibitors in the vestibule"}

\author{
Authors \\ Karin E. J. Rödström ${ }^{1, \#}$, Aytuğ K. Kiper ${ }^{2, \#, ~ W e i ~ Z h a n g ~}{ }^{1, \phi}$, Susanne Rinné2,, Ashley C. W. Pike ${ }^{1, \phi}$, \\ Matthias Goldstein ${ }^{2}$, Linus J. Conrad ${ }^{3}$, Martina Delbeck ${ }^{4}$, Michael G. Hahn ${ }^{4}$, Heinrich Meier ${ }^{4}$, \\ Magdalena Platzk ${ }^{4}$, Andrew Quigley ${ }^{1}$, David Speedman ${ }^{1}$, Leela Shrestha ${ }^{1}$, Shubhashish M.M. \\ Mukhopadhyay ${ }^{1}$, Nicola A. Burgess-Brown ${ }^{1}$, Stephen J. Tucker ${ }^{3}$, Thomas Mueller ${ }^{4}$, Niels Decher ${ }^{2, \$, *}$ \\ Elisabeth P. Carpenter ${ }^{1, \S, *}$.
}

Index:

\section{Supplementary methods}

- Synthesis of TASK inhibitors

\section{Supplementary Data}

- Supplementary Table 1. Effects of BAY1000493 on a range of ion channels

\section{Supplementary discussion}

- Structure activity relationships for the inhibitors

- Crystallographic features of the TASK-1 complexes withBAY1000493 and BAY2341237

- Disease associated variants in TASK-1, their location in the structure and predicted effects on function 


\section{Supplementary methods: Synthesis of TASK inhibitors}

\section{General methods and materials}

The ${ }^{1} \mathrm{H}-\mathrm{NMR}$ and ${ }^{13} \mathrm{C}-\mathrm{NMR}$ spectra were recorded in [D 6 ]DMSO on a Bruker Avance III HD $400 \mathrm{MHz}$ and Avance Neo $500 \mathrm{MHz}$ and are reported relative to deuterated solvent signals.

The $500 \mathrm{MHz}$ spectrometer was equipped with a $5 \mathrm{~mm}$-TCI cryoprobe, while a room temperature $5 \mathrm{~mm}$ BBI was used on the $400 \mathrm{MHz}$ magnet.

${ }^{13} \mathrm{C}-\mathrm{NMR}$ spectra were recorded on a Bruker spectrometer at $125 \mathrm{MHz}$. Chemical shift $(\delta)$ are expressed in $\mathrm{ppm}$.

Single mass HRMS spectra analysis were recorded in "Waters Time of Flight System (ToF); Electrospray Ionization (ESI)".

\section{LC-MS and HPLC methods:}

\section{Method 1 (LC-MS):}

Instrument: Waters Acquity SQD UPLC System; column: Waters Acquity UPLC HSS T3 $1.8 \mu \mathrm{m}, 50$ $\mathrm{mm} \times 1 \mathrm{~mm}$; mobile phase A: 11 of water $+0.25 \mathrm{ml}$ of $99 \%$ strength formic acid, mobile phase B: 11 of acetonitrile $+0.25 \mathrm{ml}$ of $99 \%$ strength formic acid; gradient: $0.0 \min 90 \% \mathrm{~A} \rightarrow 1.2 \min 5 \% \mathrm{~A} \rightarrow 2.0$ $\min 5 \% \mathrm{~A}$; temperature: $50^{\circ} \mathrm{C}$; flow rate: $0.40 \mathrm{ml} / \mathrm{min}$; UV detection: $208-400 \mathrm{~nm}$.

\section{Method 2(LC-MS):}

MS instrument: Thermo Scientific FT-MS; UHPLC instrument: Thermo Scientific UltiMate 3000; column: Waters HSS T3 C18 $1.8 \mu \mathrm{m}, 75 \mathrm{~mm}$ x $2.1 \mathrm{~mm}$; mobile phase A: 11 of water $+0.01 \%$ formic acid, mobile phase B: 11 of acetonitrile $+0.01 \%$ formic acid; gradient: 0.0 min $10 \% \mathrm{~B} \rightarrow 2.5 \mathrm{~min} 95 \%$ $\mathrm{B} \rightarrow 3.5 \mathrm{~min} 95 \% \mathrm{~B}$; temperature: $50^{\circ} \mathrm{C}$; flow rate: $0.90 \mathrm{ml} / \mathrm{min}$; UV detection: $210-300 \mathrm{~nm}$.

\section{Method 3 (LC-MS):}

MS instrument: Waters Micromass QM; HPLC instrument: Agilent 1100 Serie; column: Agilent ZORBAX Extend-C18 $3.5 \mu \mathrm{m}, 50 \mathrm{~mm}$ x $3.0 \mathrm{~mm}$; mobile phase A: 11 of water $+0.01 \mathrm{~mol}$ ammonium carbonate, mobile phase B: 11 of acetonitrile; gradient: $0.0 \min 98 \% \mathrm{~A} \rightarrow 0.2 \mathrm{~min} 98 \% \mathrm{~A} \rightarrow 3.0 \mathrm{~min}$ $5 \% \mathrm{~A} \rightarrow 4.5 \mathrm{~min} 5 \% \mathrm{~A}$; temperature: $40^{\circ} \mathrm{C}$; flow rate: $1.75 \mathrm{ml} / \mathrm{min}$; UV detection: $210 \mathrm{~nm}$.

\section{Method 4 (LC-MS):}

MS instrument: Waters Micromass Quattro Micro; HPLC instrument: Waters UPLC Acquity; column: Waters BEH C18 $1.7 \mu \mathrm{m}, 50 \mathrm{~mm}$ x $2.1 \mathrm{~mm}$; mobile phase A: 11 of water $+0.01 \mathrm{~mol}$ ammonium formate, mobile phase B: 11 of acetonitrile; gradient: $0.0 \min 95 \% \mathrm{~A} \rightarrow 0.1 \min 95 \% \mathrm{~A} \rightarrow 2.0 \min 15 \% \mathrm{~A} \rightarrow$ 
$2.5 \min 15 \% \mathrm{~A} \rightarrow 2.51 \mathrm{~min} 10 \% \mathrm{~A} \rightarrow 3.0 \mathrm{~min} 10 \% \mathrm{~A}$; temperature: $40^{\circ} \mathrm{C}$; flow rate: $0.5 \mathrm{ml} / \mathrm{min}$; UV detection: $210 \mathrm{~nm}$.

\section{Method 5 (LC-MS):}

Instrument: Agilent MS Quad 6150 with HPLC Agilent 1290; column: Waters Acquity UPLC HSS T3 $1.8 \mu \mathrm{m}, 50 \mathrm{~mm} \times 2.1 \mathrm{~mm}$; mobile phase A: 11 of water $+0.25 \mathrm{ml}$ of $99 \%$ strength formic acid, mobile phase B: 11 of acetonitrile $+0.25 \mathrm{ml}$ of $99 \%$ strength formic acid; gradient: $0.0 \mathrm{~min} 90 \% \mathrm{~A} \rightarrow 0.3 \mathrm{~min}$ $90 \% \mathrm{~A} \rightarrow 1.7 \mathrm{~min} 5 \% \mathrm{~A} \rightarrow 3.0 \mathrm{~min} 5 \% \mathrm{~A}$; flow rate: $1.20 \mathrm{ml} / \mathrm{min}$; temperature: $50^{\circ} \mathrm{C}$; UV detection: 205-305 nm.

\section{Method 6 (preparative HPLC):}

Instrument: Abimed Gilson 305; column: Reprosil C18 $10 \mu \mathrm{m}, 250 \mathrm{~mm}$ x $30 \mathrm{~mm}$; mobile phase A: water, mobile phase B: acetonitrile; gradient: $0-3 \min 10 \% \mathrm{~B}, 3-27 \mathrm{~min} 10 \% \mathrm{~B} \rightarrow 95 \% \mathrm{~B}, 27-34.5 \mathrm{~min}$ $95 \% \mathrm{~B}, 34.5-35.5 \mathrm{~min} 95 \% \mathrm{~B} \rightarrow 10 \% \mathrm{~B}, 35.5-36.5 \mathrm{~min} 10 \% \mathrm{~B}$; flow rate: $50 \mathrm{ml} / \mathrm{min}$; room temperature; UV detection: $210 \mathrm{~nm}$.

\section{Starting materials and intermediates:}

\section{$\underline{\text { Intermediate 1A }}$}

2-(4-chlorophenyl)imidazo[1,2-a]pyridine

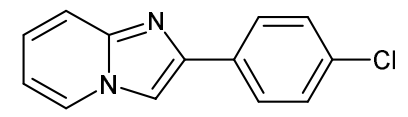

$10.95 \mathrm{~g}$ (130 mmol) of sodium bicarbonate were added to a solution of $20 \mathrm{~g}(85.65 \mathrm{mmol})$ of 2-bromo1-(4-chlorophenyl)ethanone and $8.87 \mathrm{~g}(94.22 \mathrm{mmol})$ of pyridine-2-amine in $200 \mathrm{ml}$ of ethanol, and the mixture was stirred at $80^{\circ} \mathrm{C}$ for 5 hours. The reaction was then cooled first to room temperature and then to $0^{\circ} \mathrm{C}$ (ice bath). The resulting precipitate was filtered off and washed repeatedly with an ethanol/water mixture (2:1). The solid was then dried under reduced pressure at $40^{\circ} \mathrm{C}$ overnight. This gave $19.8 \mathrm{~g}$ of the target product, which was used in the subsequent reactions without further purification.

${ }^{1} \mathrm{H}-\mathrm{NMR}\left(400 \mathrm{MHz}\right.$, DMSO- $\left.d_{6}, \delta / \mathrm{ppm}\right):$ 6.87-6.94 (m, 1H), 7.23-7.29 (m, 1H), $7.50(\mathrm{~d}, 2 \mathrm{H}), 7.58(\mathrm{~d}$, $1 \mathrm{H}), 7.99(\mathrm{~d}, 2 \mathrm{H}), 8.43(\mathrm{~s}, 1 \mathrm{H}), 8.53(\mathrm{~d}, 1 \mathrm{H})$.

LC-MS (Method 1): $\mathrm{R}_{\mathrm{t}}=0.58 \mathrm{~min} ; \mathrm{m} / \mathrm{z}=229 / 231(\mathrm{M}+\mathrm{H})^{+}$.

Analogously to intermediate $1 \mathrm{~A}$, the following compounds were prepared from the starting materials stated in each case: 


\begin{tabular}{|c|c|c|}
\hline Intermediate & Name / Structure / Starting materials & Analytical data \\
\hline $\mathbf{2 A}$ & $\begin{array}{l}\text { 2-(4-bromophenyl)imidazo[1,2-a]pyridine } \\
\text { from 2-bromo-1-(4-bromophenyl)ethanone and } \\
\text { pyridine-2-amine }\end{array}$ & $\begin{array}{l}{ }^{1} \mathrm{H}-\mathrm{NMR}\left(400 \mathrm{MHz}, \mathrm{DMSO}-d_{6},\right. \\
\delta / \mathrm{ppm}): 6.88-6.94(\mathrm{~m}, 1 \mathrm{H}), 7.23- \\
7.29(\mathrm{~m}, 1 \mathrm{H}), 7.58(\mathrm{~d}, 1 \mathrm{H}), 7.63 \\
(\mathrm{~d}, 2 \mathrm{H}), 7.92(\mathrm{~d}, 2 \mathrm{H}), 8.44(\mathrm{~s}, 1 \mathrm{H}), \\
8.53(\mathrm{~d}, 1 \mathrm{H}) . \\
\text { LC-MS (Method 1): } \\
\mathrm{R}_{\mathrm{t}}=0.63 \mathrm{~min} ; \mathrm{m} / \mathrm{z}=273 / 275 \\
(\mathrm{M}+\mathrm{H})^{+} .\end{array}$ \\
\hline $3 \mathbf{A}$ & $\begin{array}{l}\text { 2-(4-isopropylphenyl)imidazo[1,2-a]pyridine } \\
\text { from 2-bromo-1-(4-isopropylphenyl)ethanone } \\
\text { and pyridine-2-amine }\end{array}$ & $\begin{array}{l}{ }^{1} \mathrm{H}-\mathrm{NMR}\left(400 \mathrm{MHz}, \mathrm{DMSO}-d_{6},\right. \\
\delta / \mathrm{ppm}): 1.23(\mathrm{~d}, 6 \mathrm{H}), 2.85-2.96 \\
(\mathrm{~m}, 1 \mathrm{H}), 6.88(\mathrm{t}, 1 \mathrm{H}), 7.19-7.26 \\
(\mathrm{~m}, 1 \mathrm{H}), 7.31(\mathrm{~d}, 2 \mathrm{H}), 7.56(\mathrm{~d}, \\
1 \mathrm{H}), 7.88(\mathrm{~d}, 2 \mathrm{H}), 8.34(\mathrm{~s}, 1 \mathrm{H}), \\
8.51(\mathrm{~d}, 1 \mathrm{H}) . \\
\text { LC-MS }(\text { Method } 1): \\
\mathrm{R}_{\mathrm{t}}=0.68 \min ; \mathrm{m} / \mathrm{z}=237(\mathrm{M}+\mathrm{H})^{+} .\end{array}$ \\
\hline $4 \mathrm{~A}$ & $\begin{array}{l}\text { 2-(3-methoxyphenyl)imidazo[1,2-a]pyridine } \\
\text { from 2-bromo-1-(3-methoxyphenyl)ethanone } \\
\text { and pyridine-2-amine }\end{array}$ & $\begin{array}{l}{ }^{1} \mathrm{H}-\mathrm{NMR}\left(400 \mathrm{MHz}, \text { DMSO- } d_{6},\right. \\
\delta / \mathrm{ppm}): 3.83(\mathrm{~s}, 3 \mathrm{H}), 6.85-6.93 \\
(\mathrm{~m}, 2 \mathrm{H}), 7.25(\mathrm{ddd}, 1 \mathrm{H}), 7.35(\mathrm{t}, \\
1 \mathrm{H}), 7.50-7.62(\mathrm{~m}, 3 \mathrm{H}), 8.42(\mathrm{~s}, \\
1 \mathrm{H}), 8.51(\mathrm{~d}, 1 \mathrm{H}) . \\
\text { LC-MS }(\text { Method } 4): \\
\mathrm{R}_{\mathrm{t}}=1.50 \mathrm{~min} ; \mathrm{m} / \mathrm{z}=225(\mathrm{M}+\mathrm{H})^{+} .\end{array}$ \\
\hline
\end{tabular}

\section{$\underline{\text { Intermediate 5A }}$}

2-(4-chlorophenyl)imidazo[1,2-a]pyridine-3-carbaldehyde<smiles>O=Cc1c(-c2ccc(Cl)cc2)nc2ccccn12</smiles> 
$300 \mathrm{ml}$ of DMF were cooled to $0^{\circ} \mathrm{C} .44 \mathrm{ml}(470.08 \mathrm{mmol})$ of phosphorus oxychloride were then slowly added dropwise. The reaction solution was then slowly warmed to room temperature and stirred at this temperature for another hour. $43 \mathrm{~g}$ (188.03 mmol) of 2-(4-chlorophenyl)imidazo[1,2-a]pyridine were then added a little at a time. During the addition, the reaction solution warmed to $35^{\circ} \mathrm{C}$. After the addition has ended, the reaction mixture was heated to $80^{\circ} \mathrm{C}$ and stirred at this temperature for 2 hours. After cooling to room temperature, the solution was slowly added to 3 litres of ice-water. The resulting solid was filtered off with suction, washed repeatedly with water and dried in a high-vacuum drying cabinet at $40^{\circ} \mathrm{C}$ overnight. This gave $39.6 \mathrm{~g}(154.27 \mathrm{mmol}, 82 \%$ of theory) of the target product.

${ }^{1} \mathrm{H}-\mathrm{NMR}\left(400 \mathrm{MHz}, \mathrm{DMSO}-d_{6}, \delta / \mathrm{ppm}\right): 7.37$ (t, 1H), $7.63(\mathrm{~d}, 2 \mathrm{H}), 7.78(\mathrm{t}, 1 \mathrm{H}), 7.90-7.99(\mathrm{~m}, 3 \mathrm{H}), 9.58$ $(\mathrm{d}, 1 \mathrm{H}), 10.02(\mathrm{~s}, 1 \mathrm{H})$.

LC-MS (Method 1): $\mathrm{R}_{\mathrm{t}}=0.97 \mathrm{~min} ; \mathrm{m} / \mathrm{z}=257 / 259(\mathrm{M}+\mathrm{H})^{+}$.

Analogously to intermediate 5A, the following compounds were prepared from the starting material stated in each case:

\begin{tabular}{|c|c|c|}
\hline Intermediate & Name / Structure / Starting material & Analytical data \\
\hline 6A & from 2-(4-bromophenyl)imidazo[1,2-a]pyridine & $\begin{array}{l}{ }^{1} \mathrm{H}-\mathrm{NMR}\left(400 \mathrm{MHz}, \text { DMSO- } d_{6},\right. \\
\delta / \mathrm{ppm}): 7.35(\mathrm{t}, 1 \mathrm{H}), 7.72-7.80(\mathrm{~m}, \\
3 \mathrm{H}), 7.85-7.95(\mathrm{~m}, 3 \mathrm{H}), 9.58(\mathrm{~d}, \\
1 \mathrm{H}), 10.02(\mathrm{~s}, 1 \mathrm{H}) . \\
\text { LC-MS (Method 2): } \\
\mathrm{R}_{\mathrm{t}}=1.76 \mathrm{~min} ; \mathrm{m} / \mathrm{z}=301 / 303 \\
(\mathrm{M}+\mathrm{H})^{+} .\end{array}$ \\
\hline 7A & $\begin{array}{l}\text { 2-(4-isopropylphenyl)imidazo[1,2-a]pyridine-3- } \\
\text { from 2-(4-isopropylphenyl)imidazo[1,2- } \\
\text { a]pyridine }\end{array}$ & $\begin{array}{l}{ }^{1} \mathrm{H}-\mathrm{NMR}\left(400 \mathrm{MHz}, \text { DMSO- } d_{6},\right. \\
\text { S/ppm): } 1.27(\mathrm{~d}, 6 \mathrm{H}), 2.93-3.05 \\
(\mathrm{~m}, 1 \mathrm{H}), 7.33(\mathrm{t}, 1 \mathrm{H}), 7.44(\mathrm{~d}, 2 \mathrm{H}), \\
7.74(\mathrm{t}, 1 \mathrm{H}), 7.85(\mathrm{~d}, 2 \mathrm{H}), 7.91(\mathrm{~d}, \\
1 \mathrm{H}), 9.58(\mathrm{~d}, 1 \mathrm{H}), 10.03(\mathrm{~s}, 1 \mathrm{H}) . \\
\text { LC-MS (Method 1): } \\
\mathrm{R}_{\mathrm{t}}=1.03 \mathrm{~min} ; \mathrm{m} / \mathrm{z}=265(\mathrm{M}+\mathrm{H})^{+} .\end{array}$ \\
\hline
\end{tabular}




\begin{tabular}{|c|c|c|}
\hline Intermediate & Name / Structure / Starting material & Analytical data \\
\hline 8A & $\begin{array}{l}\text { 2-(3-methoxyphenyl)imidazo[1,2-a]pyridine-3- } \\
\text { from 2-(3-methoxyphenyl)imidazo[1,2- } \\
\text { a]pyridine }\end{array}$ & $\begin{array}{l}{ }^{1} \mathrm{H}-\mathrm{NMR}\left(400 \mathrm{MHz}, \text { DMSO- } d_{6},\right. \\
\delta / \mathrm{ppm}): 3.86(\mathrm{~s}, 3 \mathrm{H}), 7.09-7.17 \\
(\mathrm{~m}, 1 \mathrm{H}), 7.36(\mathrm{t}, 1 \mathrm{H}), 7.43-7.52 \\
(\mathrm{~m}, 3 \mathrm{H}), 7.77(\mathrm{t}, 1 \mathrm{H}), 7.93(\mathrm{~d}, 1 \mathrm{H}), \\
9.59(\mathrm{~d}, 1 \mathrm{H}), 10.03(\mathrm{~s}, 1 \mathrm{H}) . \\
\text { LC-MS (Method 2): } \\
\mathrm{R}_{\mathrm{t}}=1.52 \mathrm{~min} ; \mathrm{m} / \mathrm{z}=253(\mathrm{M}+\mathrm{H})^{+} .\end{array}$ \\
\hline
\end{tabular}

\section{Intermediate 9A}

tert-butyl 4-\{[2-(4-chlorophenyl)imidazo[1,2-a]pyridin-3-yl]methyl\}piperazine-1-carboxylate

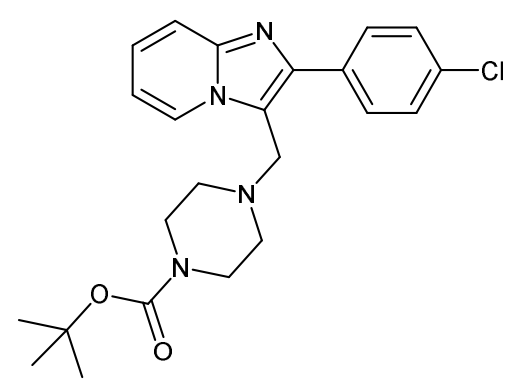

Under argon and at room temperature, $1 \mathrm{~g}$ (3.90 mmol) of 2-(4-chlorophenyl)imidazo[1,2-a]pyridine-3carbaldehyde was dissolved in $20 \mathrm{ml}$ of THF, and $1.45 \mathrm{~g}(7.79 \mathrm{mmol})$ of tert-butyl piperazine-1carboxylate and $0.45 \mathrm{ml}(7.79 \mathrm{mmol})$ of acetic acid were added. Subsequently, $2.48 \mathrm{~g}$ of sodium triacetoxyborohydride $(11.69 \mathrm{mmol})$ was added in a little at a time, and the reaction mixture was stirred at room temperature overnight. After the reaction had ended, water was slowly and carefully added dropwise (evolution of gas), and ethyl acetate was then added. The organic phase was removed and the aqueous phase was extracted twice with ethyl acetate. The combined organic phases were dried over magnesium sulphate, filtered and concentrated to dryness under reduced pressure on a rotary evaporator. The resulting residue was applied to silica gel and purified by column chromatography on silica gel (mobile phase: cyclohexane/ethyl acetate 2:1). This gave $1.27 \mathrm{~g} \mathrm{(2.97} \mathrm{mmol,} 76 \%$ of theory) of the target compound.

${ }^{1} \mathrm{H}-\mathrm{NMR}$ (400 MHz, DMSO- $\left.d_{6}, \delta / \mathrm{ppm}\right): 1.38$ (s, 9H), 2.31-2.44 (m, 4H), 3.22-3.32 (m, 4H, partially hidden by water signal), 3.99 (s, 2H), $6.98(\mathrm{t}, 1 \mathrm{H}), 7.31(\mathrm{t}, 1 \mathrm{H}), 7.53(\mathrm{~d}, 2 \mathrm{H}), 7.61(\mathrm{~d}, 1 \mathrm{H}), 7.92(\mathrm{~d}, 2 \mathrm{H})$, $8.56(\mathrm{~d}, 1 \mathrm{H})$. 
LC-MS (Method 1): $\mathrm{R}_{\mathrm{t}}=0.85 \mathrm{~min} ; \mathrm{m} / \mathrm{z}=427 / 429(\mathrm{M}+\mathrm{H})^{+}$.

Analogously to intermediate 9A, the following compounds were prepared from the starting materials stated in each case:

\begin{tabular}{|c|c|c|}
\hline Intermediate & Name / Structure / Starting materials & Analytical data \\
\hline 10A & $\begin{array}{l}\text { tert-butyl 4-\{[2-(4-bromophenyl)imidazo[1,2- } \\
\text { a]pyridin-3-yl]methyl }\} \text { piperazine-1-carboxylate } \\
\text { carbaldehyde and tert-butyl piperazine-1- } \\
\text { carboxylate }\end{array}$ & 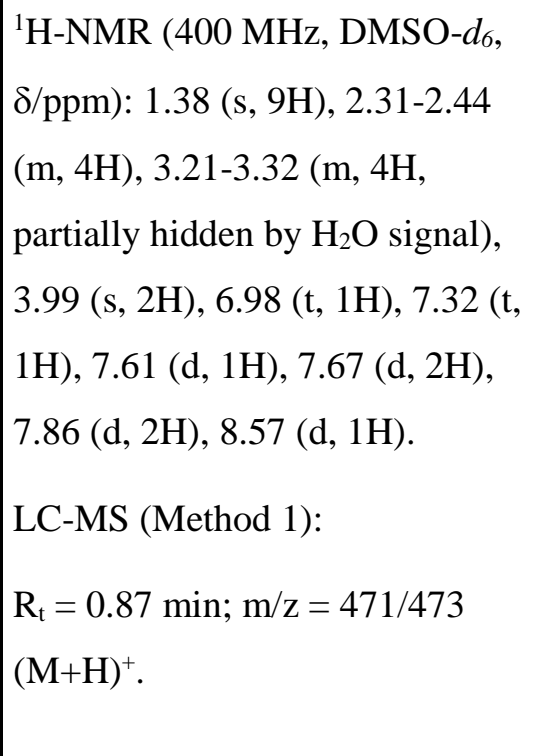 \\
\hline 11A & $\begin{array}{l}\text { tert-butyl 4-\{[2-(4-isopropylphenyl)imidazo[1,2- } \\
\text { a]pyridin-3-yl]methyl\}piperazine-1-carboxylate } \\
\text { a]pyridine-3-carbaldehyde and tert-butyl } \\
\text { piperazine-1-carboxylate }\end{array}$ & 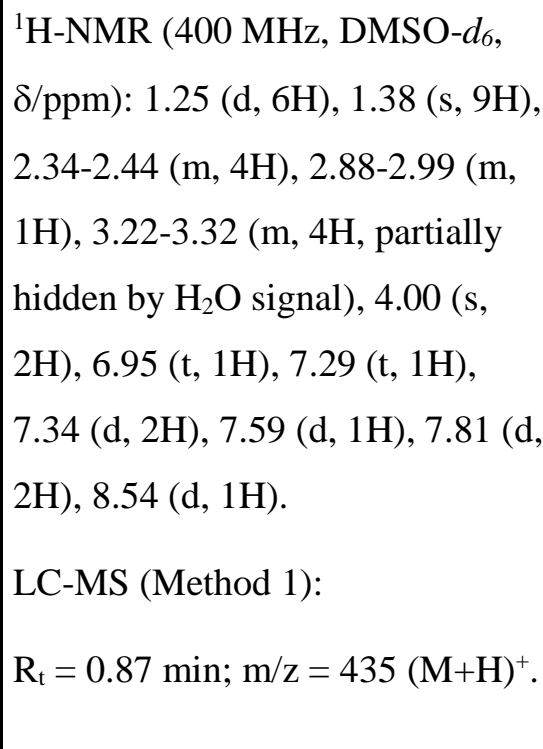 \\
\hline
\end{tabular}




\begin{tabular}{|c|c|c|}
\hline Intermediate & Name / Structure / Starting materials & Analytical data \\
\hline $12 \mathrm{~A}$ & $\begin{array}{l}\text { tert-butyl 4-\{[2-(3-methoxyphenyl)imidazo[1,2- } \\
\text { a]pyridin-3-yl]methyl }\} \text { piperazine-1-carboxylate } \\
\text { from 2-(3-methoxyphenyl)imidazo[1,2- } \\
\text { a]pyridine-3-carbaldehyde and tert-butyl } \\
\text { piperazine-1-carboxylate }\end{array}$ & 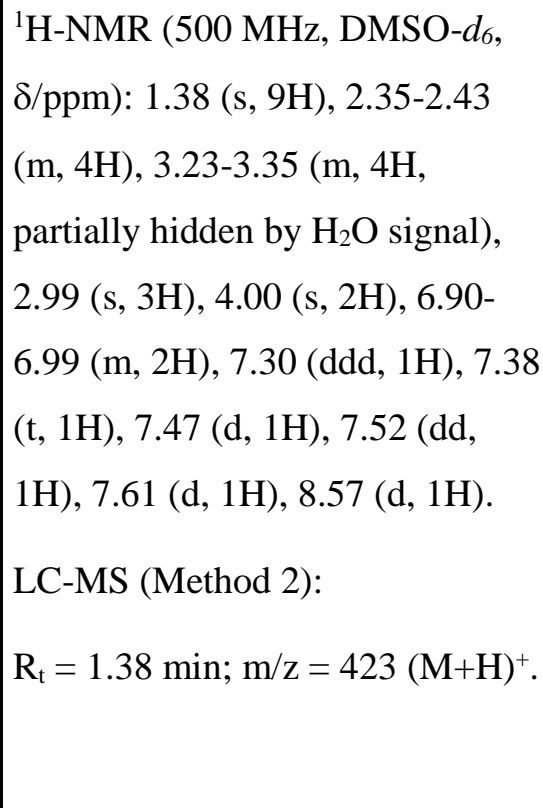 \\
\hline
\end{tabular}

\section{$\underline{\text { Intermediate 14A }}$}

2-(4-chlorophenyl)-3-(piperazin-1-ylmethyl)imidazo[1,2-a]pyridine dihydrochloride

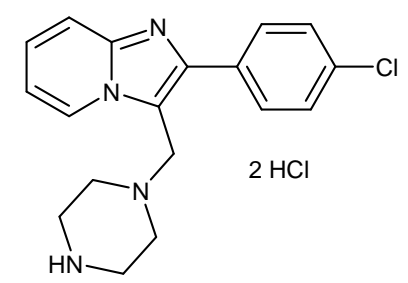

With stirring, $41.11 \mathrm{ml}$ of a $4 \mathrm{M}$ solution of hydrogen chloride in dioxane were added to $2.34 \mathrm{~g}$ (5.48 mmol) of tert-butyl 4-\{[2-(4-chlorophenyl)imidazo[1,2-a]pyridin-3-yl]methyl\}piperazine-1carboxylate, and the mixture was stirred at room temperature for 3 hours. The solid obtained was then filtered off with suction, washed repeatedly with diethyl ether and dried under high vacuum at $40^{\circ} \mathrm{C}$. This gave $2.48 \mathrm{~g}$ of the target product, which was used in the subsequent reactions without further purification.

LC/MS (Method 1): $\mathrm{R}_{\mathrm{t}}=0.35 \mathrm{~min} ; \mathrm{m} / \mathrm{z}=327 / 329(\mathrm{M}+\mathrm{H})^{+}$.

Analogously to intermediate $14 \mathrm{~A}$, the following compounds were prepared from the starting material stated in each case: 


\begin{tabular}{|c|c|c|}
\hline Intermediate & Name / Structure / Starting material & Analytical data \\
\hline 16A & $\begin{array}{l}\text { 2-(4-bromophenyl)-3-(piperazin-1- } \\
\text { ylmethyl)imidazo[1,2-a]pyridine dihydrochloride } \\
\text { bromophenyl)imidazo[1,2-a]pyridin-3- } \\
\text { yl]methyl }\} \text { piperazine-1-carboxylate }\end{array}$ & $\begin{array}{l}\text { LC-MS (Method } 1): \\
\mathrm{R}_{\mathrm{t}}=0.38 \mathrm{~min} ; \mathrm{m} / \mathrm{z}=371 / 373 \\
(\mathrm{M}+\mathrm{H})^{+}\end{array}$ \\
\hline 17A & $\begin{array}{l}\text { 2-(4-isopropylphenyl)-3-(piperazin-1- } \\
\text { ylmethyl)imidazo[1,2-a]pyridine dihydrochloride } \\
\text { isopropylphenyl)imidazo[1,2-a]pyridin-3- } \\
\text { yl]methyl }\} \text { piperazine-1-carboxylate }\end{array}$ & $\begin{array}{l}\text { LC-MS (Method 1): } \\
\mathrm{R}_{\mathrm{t}}=0.46 \mathrm{~min} ; \mathrm{m} / \mathrm{z}=335(\mathrm{M}+\mathrm{H})^{+}\end{array}$ \\
\hline 18A & $\begin{array}{l}\text { 2-(3-methoxyphenyl)-3-(piperazin-1- } \\
\text { ylmethyl)imidazo[1,2-a]pyridine dihydrochloride } \\
\text { methoxyphenyl)imidazo[1,2-a]pyridin-3- } \\
\text { yl]methyl\}piperazine-1-carboxylate }\end{array}$ & $\begin{array}{l}\text { LC-MS (Method 4): } \\
\mathrm{R}_{\mathrm{t}}=1.12 \mathrm{~min} ; \mathrm{m} / \mathrm{z}=322(\mathrm{M}+\mathrm{H})^{+} .\end{array}$ \\
\hline
\end{tabular}




\section{$\underline{\text { Intermediate 19A }}$}

2-(4-bromophenyl)-3-(piperazin-1-ylmethyl)imidazo[1,2-a]pyridine

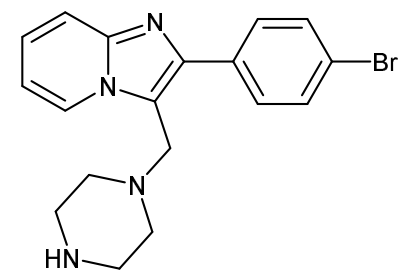

$8.60 \mathrm{~g} \quad$ (19.36 mmol) of 2-(4-bromophenyl)-3-(piperazin-1-ylmethyl)imidazo[1,2-a]pyridine dihydrochloride were dissolved in $83 \mathrm{ml}$ of THF, $13.5 \mathrm{ml}(97 \mathrm{mmol})$ of triethylamine were added and the mixture was stirred at room temperature for 2 hours. Water and ethyl acetate were then added to the reaction solution. The organic phase was separated off and the aqueous phase was extracted ten times with dichloromethane. The combined organic phases were dried over magnesium sulphate, filtered and concentrated to dryness under reduced pressure on a rotary evaporator. This gave $3.15 \mathrm{~g}(8.48 \mathrm{mmol}$, $44 \%$ of theory) of the target product, which was used in the subsequent reactions without further purification.

${ }^{1} \mathrm{H}-\mathrm{NMR}$ (400 MHz, DMSO-d 6 , $\left.\delta / \mathrm{ppm}\right)$ : 2.29-2.43 (m, 4H), 2.61-2.75 (m, 4H), 3.95 (s, 2H), 6.98 (t, $1 \mathrm{H}), 7.31(\mathrm{t}, 1 \mathrm{H}), 7.60(\mathrm{~d}, 1 \mathrm{H}), 7.67(\mathrm{~d}, 2 \mathrm{H}), 7.85(\mathrm{~d}, 2 \mathrm{H}), 8.54(\mathrm{~d}, 1 \mathrm{H})$.

LC-MS (Method 2): $\mathrm{R}_{\mathrm{t}}=0.59 \mathrm{~min} ; \mathrm{m} / \mathrm{z}=371 / 373(\mathrm{M}+\mathrm{H})^{+}$.

\section{Working compounds:}

\section{Compound 1}

(4-\{[2-(4-chlorophenyl)imidazo[1,2-a]pyridin-3-yl]methyl\}piperazin-1-yl)(cyclopentyl)methanone<smiles>O=C(C1CCCC1)N1CCN(Cc2c(-c3ccc(Cl)cc3)nc3ccccn23)CC1</smiles> 


\section{Synthesis method 1}

$150 \mathrm{mg} \quad(0.38 \mathrm{mmol}) \quad$ of 2-(4-chlorophenyl)-3-(piperazin-1-ylmethyl)imidazo[1,2-a]pyridine dihydrochloride were dissolved in $2 \mathrm{ml}$ of DMF, and $261 \mu \mathrm{l}(1.50 \mathrm{mmol})$ of $N, N$-diisopropylethylamine were added. The mixture was stirred at room temperature for $30 \mathrm{~min} .47 \mathrm{mg}(0.41 \mathrm{mmol})$ of cyclopentanecarboxylic acid and $214 \mathrm{mg}(0.56 \mathrm{mmol})$ of 2-(7-aza-1H-benzotriazol-1-yl)-1,1,3,3tetramethyluronium hexafluorophosphate (HATU) were then added, and the mixture was stirred further at room temperature overnight. The reaction mixture was then separated directly into its components by preparative HPLC (Method 6). This gave $110 \mathrm{mg}$ ( $0.26 \mathrm{mmol}, 69 \%$ of theory) of the title compound.

LC-MS (Method 1): $\mathrm{R}_{\mathrm{t}}=0.80 \mathrm{~min} ; \mathrm{m} / \mathrm{z}=423 / 425(\mathrm{M}+\mathrm{H})^{+}$.

HRMS calculated for $\mathrm{C}_{24} \mathrm{H}_{27} \mathrm{ClN}_{4} \mathrm{O}+\left[\mathrm{H}^{+}\right]$: 423.1952; found: 423.1954 .

${ }^{1} \mathrm{H}-\mathrm{NMR}\left(400 \mathrm{MHz}, \mathrm{DMSO}-d_{6}\right): \delta[\mathrm{ppm}]=1.42-1.78(\mathrm{~m}, 8 \mathrm{H}), 2.34-2.46(\mathrm{~m}, 4 \mathrm{H}), 2.88-2.99(\mathrm{~m}, 1 \mathrm{H})$, 3.37-3.50 (m, 4H), $4.01(\mathrm{~s}, 2 \mathrm{H}), 6.98(\mathrm{t}, 1 \mathrm{H}), 7.32(\mathrm{t}, 1 \mathrm{H}), 7.53(\mathrm{~d}, 2 \mathrm{H}), 7.61(\mathrm{~d}, 1 \mathrm{H}), 7.92(\mathrm{~d}, 2 \mathrm{H}), 8.58$ $(\mathrm{d}, 1 \mathrm{H})$.

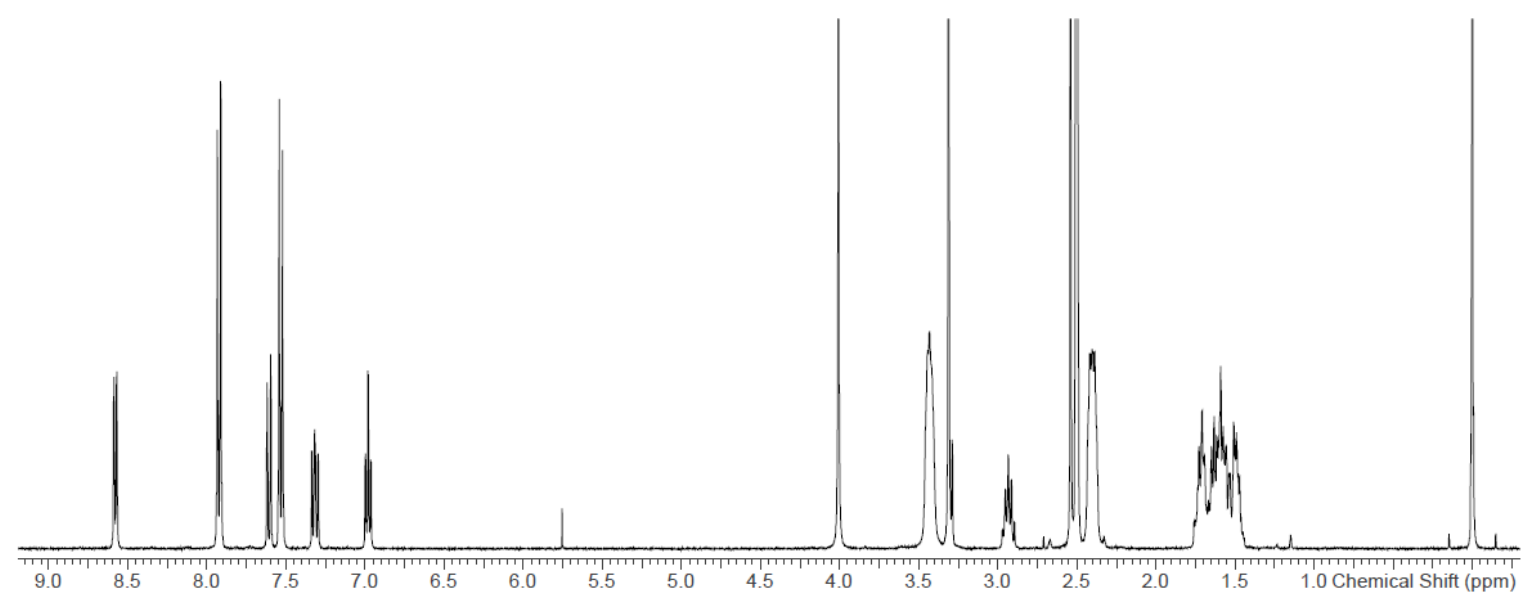

${ }^{13} \mathrm{C}-\mathrm{NMR}\left(125 \mathrm{MHz}, \mathrm{DMSO}-d_{6}\right): \delta[\mathrm{ppm}]=25.5,29.5,39.9,41.2$ 44.8, 50.1, 52.0, 52.5, 112.1, 116.6, $116.8,125.0,125.7,128.5,129.9,132.2,133.5,142.6,144.2,173.5$. 


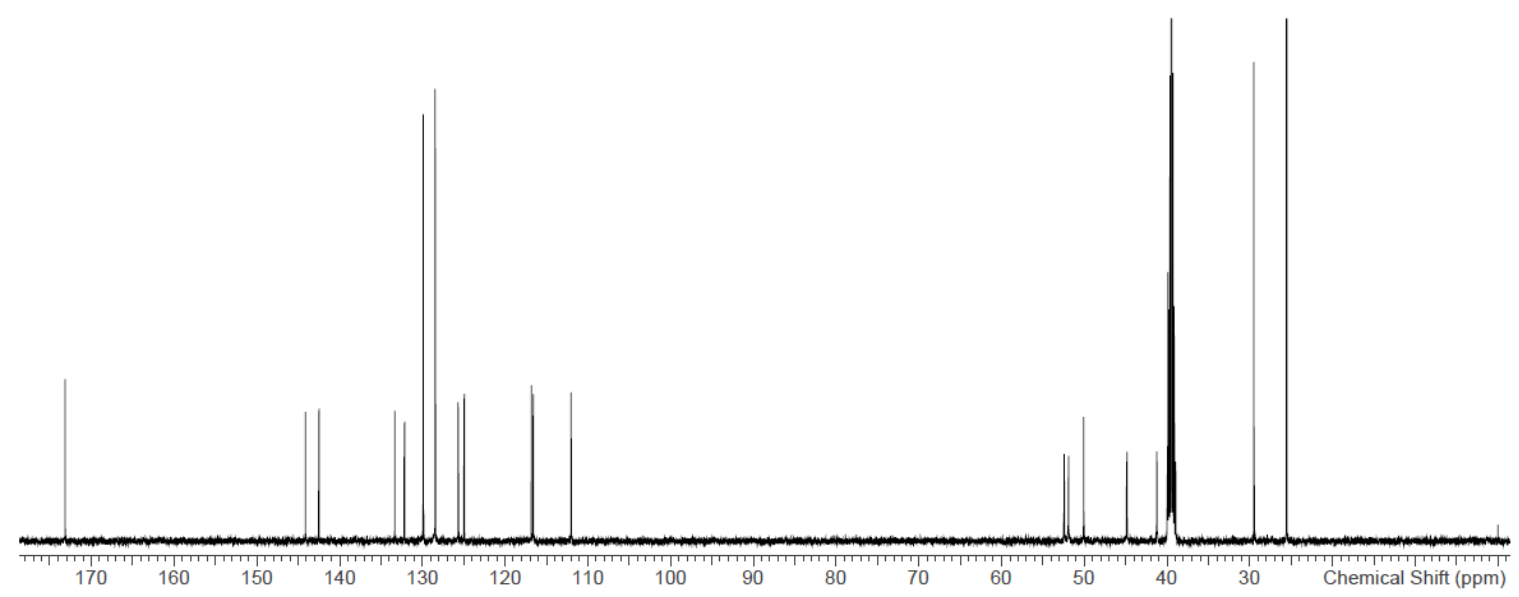

\section{Compound 2}

(4-\{[2-(4-bromophenyl)imidazo[1,2-a]pyridin-3-yl]methyl\}piperazin-1-yl)(cyclopentyl)methanone<smiles>O=C(C1CCCC1)N1CCN(Cc2c(-c3ccc(Br)cc3)nc3ccccn23)CC1</smiles>

\section{Synthesis method 2}

$100 \mathrm{mg}(0.27 \mathrm{mmol})$ of 2-(4-bromophenyl)-3-(piperazin-1-ylmethyl)imidazo[1,2-a]pyridine and $35 \mu \mathrm{l}$ $(0.32 \mathrm{mmol})$ of cyclopentanecarboxylic acid were dissolved in $2 \mathrm{ml}$ of DMF, and $133 \mathrm{mg}(0.35 \mathrm{mmol})$ of 2-(7-aza-1H-benzotriazol-1-yl)-1,1,3,3-tetramethyluronium hexafluorophosphate (HATU) and $94 \mu 1$ $(0.54 \mathrm{mmol})$ of $N, N$-diisopropylethylamine were added. The mixture was stirred at room temperature overnight. The reaction mixture was then separated directly into its components by preparative HPLC (Method 6). This gave $61 \mathrm{mg}$ ( $0.13 \mathrm{mmol}, 48 \%$ of theory) of the title compound.

LC-MS (Method 1): $\mathrm{R}_{\mathrm{t}}=0.81 \mathrm{~min} ; \mathrm{m} / \mathrm{z}=467 / 469(\mathrm{M}+\mathrm{H})^{+}$.

HRMS calculated for $\mathrm{C}_{24} \mathrm{H}_{27} \mathrm{BrN}_{4} \mathrm{O}+\left[\mathrm{H}^{+}\right]$: 467.1446; found: 467.1447 .

${ }^{1} \mathrm{H}-\mathrm{NMR}\left(400 \mathrm{MHz}, \mathrm{DMSO}-d_{6}\right): \delta[\mathrm{ppm}]=1.42-1.77(\mathrm{~m}, 8 \mathrm{H}), 2.35-2.46(\mathrm{~m}, 4 \mathrm{H}), 2.87-2.99(\mathrm{~m}, 1 \mathrm{H})$, 3.36-3.50 (m, 4H), $4.00(\mathrm{~s}, 2 \mathrm{H}), 6.98(\mathrm{t}, 1 \mathrm{H}), 7.32(\mathrm{t}, 1 \mathrm{H}), 7.61(\mathrm{~d}, 1 \mathrm{H}), 7.67(\mathrm{~d}, 2 \mathrm{H}), 7.86(\mathrm{~d}, 2 \mathrm{H}), 8.58$ $(d, 1 H)$. 


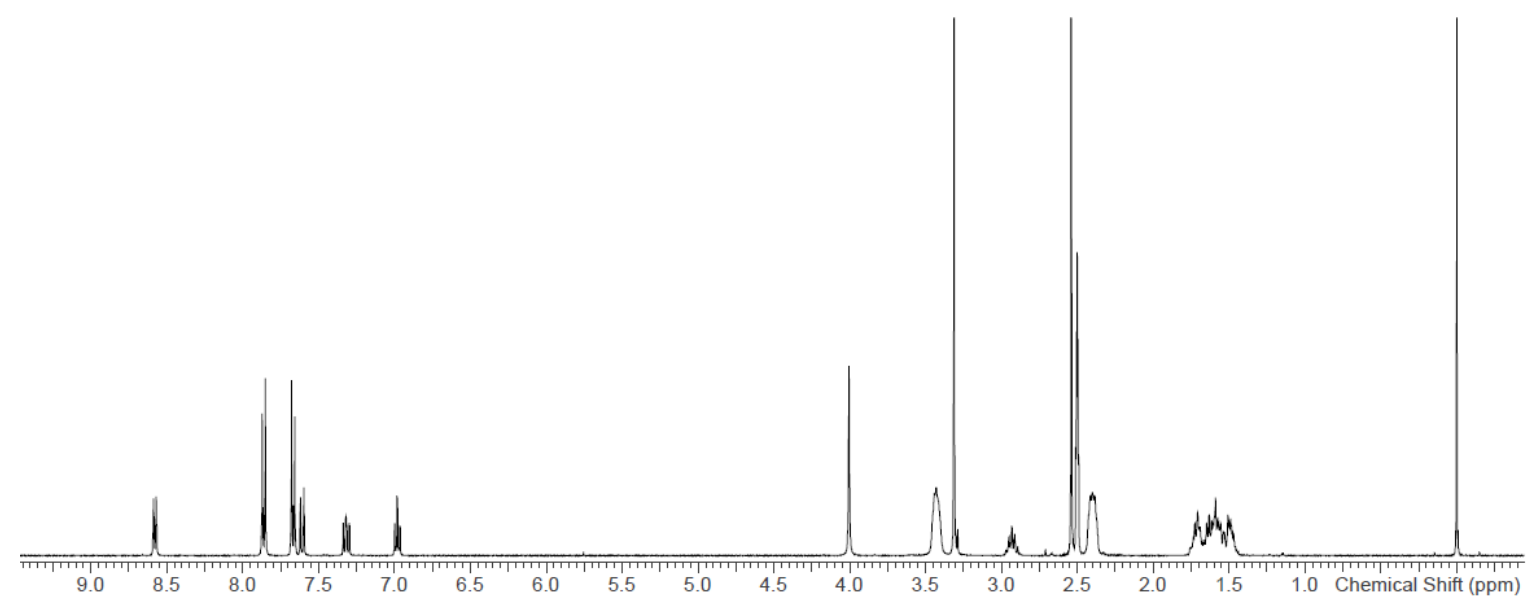

${ }^{13} \mathrm{C}-\mathrm{NMR}\left(125 \mathrm{MHz}, \mathrm{DMSO}-d_{6}\right): \delta[\mathrm{ppm}]=25.6,29.5,39.8,41.2,44.8,50.1,51.9,52.4,111.9,116.5$, $116.8,120.8,125.0,125.6,130.2,131.3,133.8,142.5,144.2,173.2$.

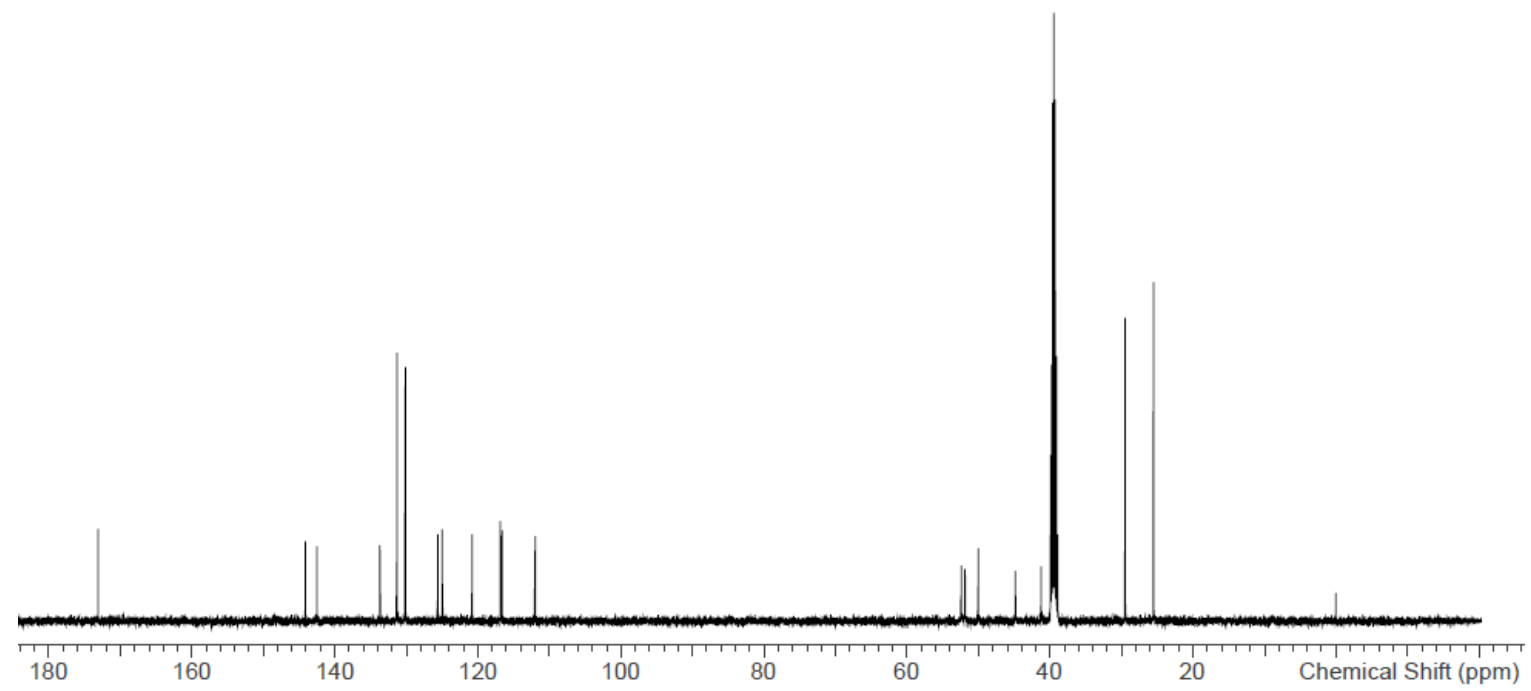

\section{Compound 3 - BAY100493}

(4-\{[2-(4-bromophenyl)imidazo[1,2-a]pyridin-3-yl]methyl\}piperazin-1-yl)(2-fluorophenyl)methanone<smiles>O=C(c1ccccc1F)N1CCN(Cc2c(-c3ccc(Br)cc3)nc3ccccn23)CC1</smiles> 


\section{Synthesis method 3}

$1.77 \mathrm{~g} \quad$ (3.98 mmol) of 2-(4-bromophenyl)-3-(piperazin-1-ylmethyl)imidazo[1,2-a]pyridine dihydrochloride were taken up in $30 \mathrm{ml}$ of dichloromethane, $2.77 \mathrm{ml}(15.90 \mathrm{mmol})$ of $\mathrm{N}, \mathrm{N}$ diisopropylethylamine were added and the mixture was stirred at room temperature for $30 \mathrm{~min}$. After cooling of the reaction solution to $0^{\circ} \mathrm{C}, 0.52 \mathrm{ml}(4.37 \mathrm{mmol})$ of 2-fluorobenzoyl chloride was added dropwise, and the mixture was then warmed to room temperature and stirred at this temperature overnight. After addition of water, the solution was shaken in a separating funnel and the phases were separated. The organic phase was dried over magnesium sulphate, filtered and concentrated to dryness under reduced pressure on a rotary evaporator. The resulting residue was applied to silica gel and purified by column chromatography on silica gel (Biotage; mobile phase: cyclohexane/ethyl acetate 4:1). This gave $1.42 \mathrm{~g}$ ( $2.88 \mathrm{mmol}, 72 \%$ of theory) of the target compound.

LC-MS (Method 1): $\mathrm{R}_{\mathrm{t}}=0.74 \mathrm{~min} ; \mathrm{m} / \mathrm{z}=493 / 495(\mathrm{M}+\mathrm{H})^{+}$.

HRMS calculated for $\mathrm{C}_{25} \mathrm{H}_{22} \mathrm{BrFN}_{4} \mathrm{O}+\left[\mathrm{H}^{+}\right]$: 493.1039; found: 493.1042 .

${ }^{1} \mathrm{H}-\mathrm{NMR}\left(400 \mathrm{MHz}, \mathrm{DMSO}-d_{6}\right): \delta[\mathrm{ppm}]=2.36-2.45(\mathrm{~m}, 2 \mathrm{H}), 2.47-2.59(\mathrm{~m}, 2 \mathrm{H}$, hidden by DMSO signal), 3.13-3.23 (m, 2H), 3.56-3.68 (m, 2H), $4.03(\mathrm{~s}, 2 \mathrm{H}), 6.98(\mathrm{t}, 1 \mathrm{H}), 7.23-7.41(\mathrm{~m}, 4 \mathrm{H}), 7.44-7.53$ (m, 1H), $7.60(\mathrm{~d}, 1 \mathrm{H}), 7.67(\mathrm{~d}, 2 \mathrm{H}), 7.85(\mathrm{~d}, 2 \mathrm{H}), 8.58(\mathrm{~d}, 1 \mathrm{H})$.

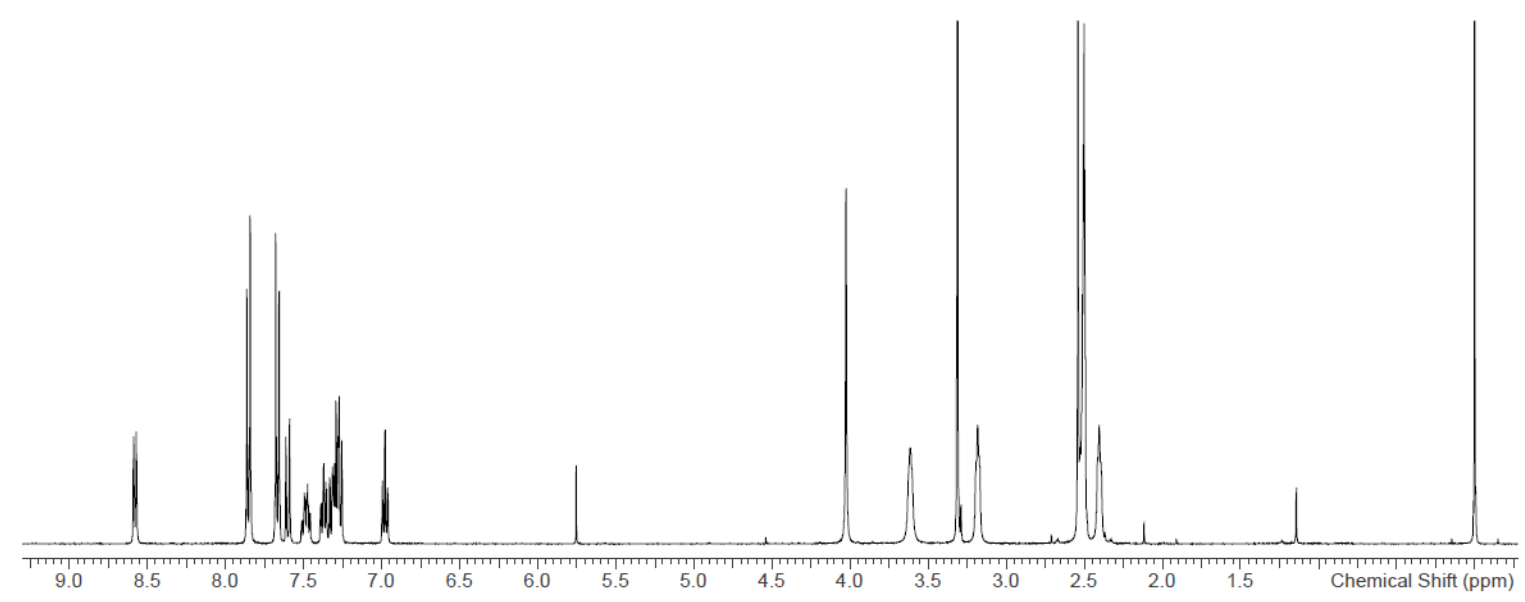

${ }^{13} \mathrm{C}-\mathrm{NMR}\left(125 \mathrm{MHz}, \mathrm{DMSO}-d_{6}\right): \delta[\mathrm{ppm}]=41.2,46.4,49.9,51.6,52.1,111.1,115.7,116.5,116.7$, $120.7,123.9,124.8,125.0,125.6,128.7,130.1,131.3$ 131.3, 133.8, 142.7, 144.4, 157.6, 163.9. 


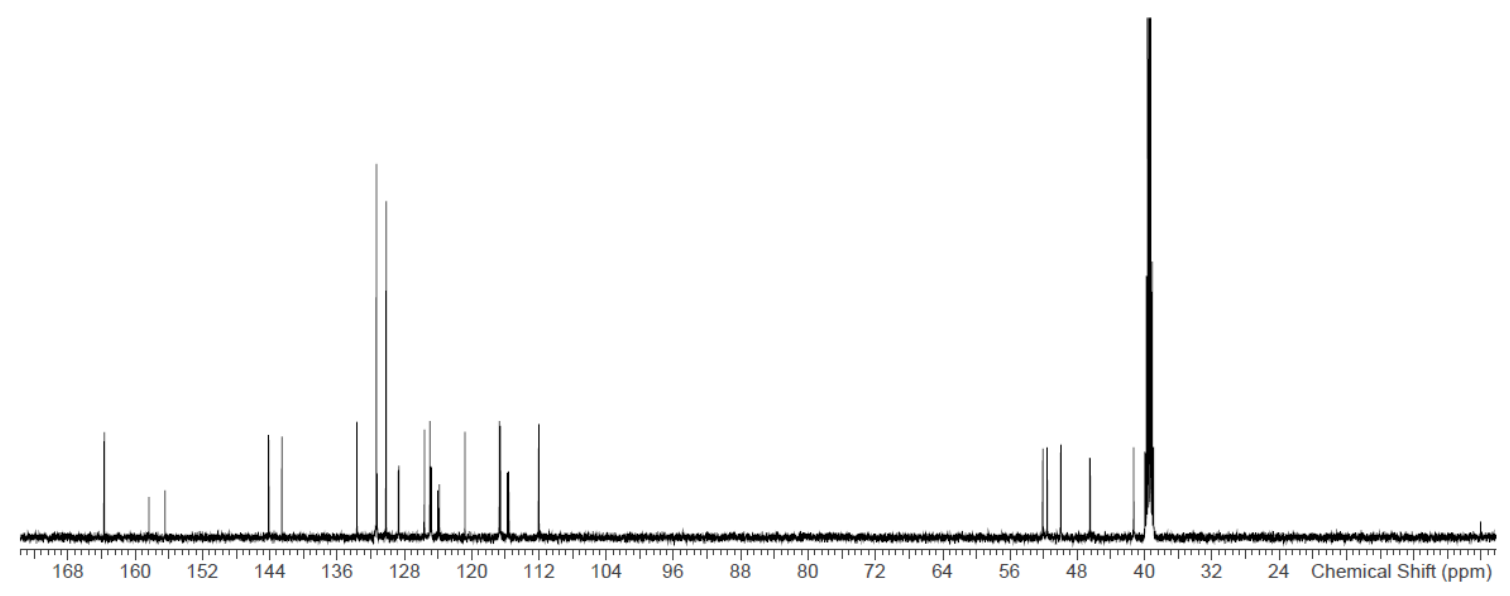

\section{Compound 6 - BAY2341237}

(4-\{[2-(4-chlorophenyl)imidazo[1,2-a]pyridin-3-yl]methyl\}piperazin-1-yl)[6-

(trifluoromethoxy)pyridin-2-yl]methanone

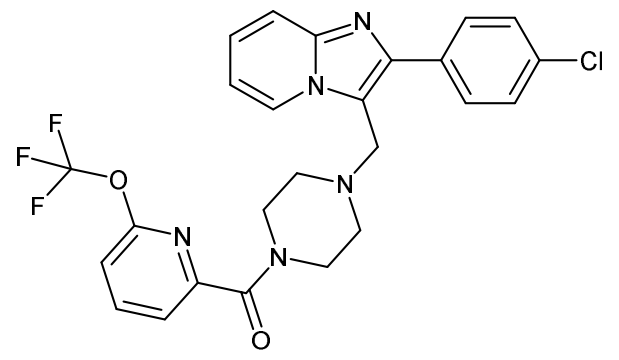

\section{Synthesis method 4}

$75 \mathrm{mg}$ ( $0.36 \mathrm{mmol})$ of 6-(trifluoromethoxy)pyridine-2-carboxylic acid were dissolved in $1.8 \mathrm{ml}$ of DMF, $148 \mathrm{mg} \quad(0.39 \mathrm{mmol}) \quad$ of 2 -(7-aza-1H-benzotriazol-1-yl)-1,1,3,3-tetramethyluronium hexafluorophosphate (HATU) were added and the mixture was stirred at room temperature for $30 \mathrm{~min}$. $120 \mathrm{mg} \quad(0.30 \mathrm{mmol})$ of 2-(4-chlorophenyl)-3-(piperazin-1-ylmethyl)imidazo[1,2-a]pyridine dihydrochloride and $0.16 \mathrm{ml}(0.90 \mathrm{mmol})$ of $N, N$-diisopropylethylamine were then added, and the mixture was stirred further at room temperature overnight. The reaction mixture was then separated directly into its components by preparative HPLC (Method 6). This gave $112 \mathrm{mg}(0.22 \mathrm{mmol}, 73 \%$ of theory) of the title compound.

LC-MS (Method 2): $\mathrm{R}_{\mathrm{t}}=1.55 \mathrm{~min} ; \mathrm{m} / \mathrm{z}=516 / 518(\mathrm{M}+\mathrm{H})^{+}$.

HRMS calculated for $\mathrm{C}_{25} \mathrm{H}_{21} \mathrm{ClF}_{3} \mathrm{~N}_{5} \mathrm{O}_{2}+\left[\mathrm{H}^{+}\right]$: 516.1414; found: 516.1416 .

${ }^{1} \mathrm{H}-\mathrm{NMR}\left(400 \mathrm{MHz}, \mathrm{DMSO}-d_{6}\right): \delta[\mathrm{ppm}]=2.44$ (br. t, 2H), 2.55 (br. t, 2H), 3.37 (br. t, 2H), 3.61 (br. s, 2H), $4.05(\mathrm{~s}, 2 \mathrm{H}), 6.98(\mathrm{td}, 1 \mathrm{H}), 7.32(\mathrm{ddd}, 1 \mathrm{H}), 7.38(\mathrm{~d}, 1 \mathrm{H}), 7.53(\mathrm{~d}, 2 \mathrm{H}), 7.58-7.65(\mathrm{~m}, 2 \mathrm{H}), 7.91(\mathrm{~d}$, $2 \mathrm{H}), 8.14(\mathrm{t}, 1 \mathrm{H}), 8.58(\mathrm{~d}, 1 \mathrm{H})$. 


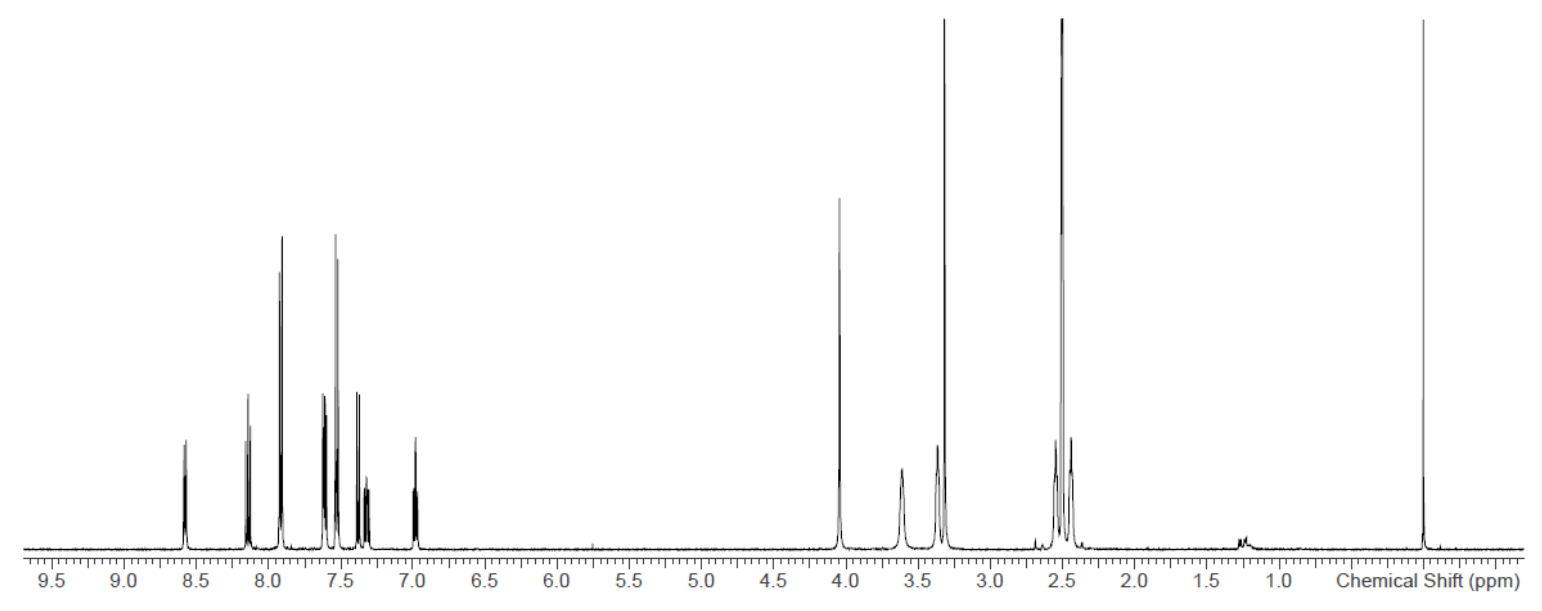

${ }^{13} \mathrm{C}-\mathrm{NMR}\left(125 \mathrm{MHz}, \mathrm{DMSO}-d_{6}\right): \delta[\mathrm{ppm}]=41.7,46.4,49.9,51.7,52.1,112.3,113.9,116.4,116.7$, 119.5, 122.2, 125.4, 125.8, 128.5, 129.9, 132.2, 132.5, 142.5, 142.7, 144.1, 151.7, 154.3, 164.6.

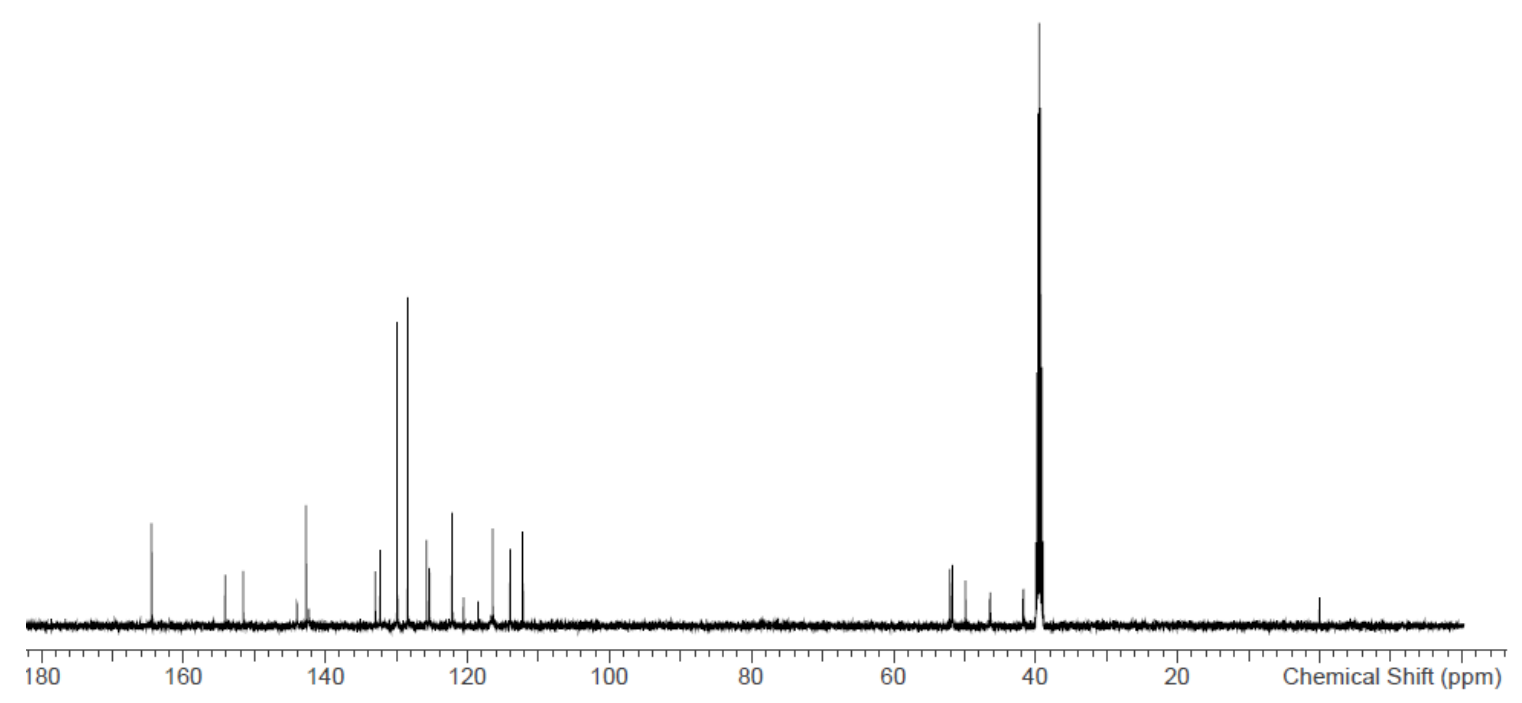

The following compounds were also prepared according to the Synthesis methods 1-4 described above, using the starting materials stated in each case: 


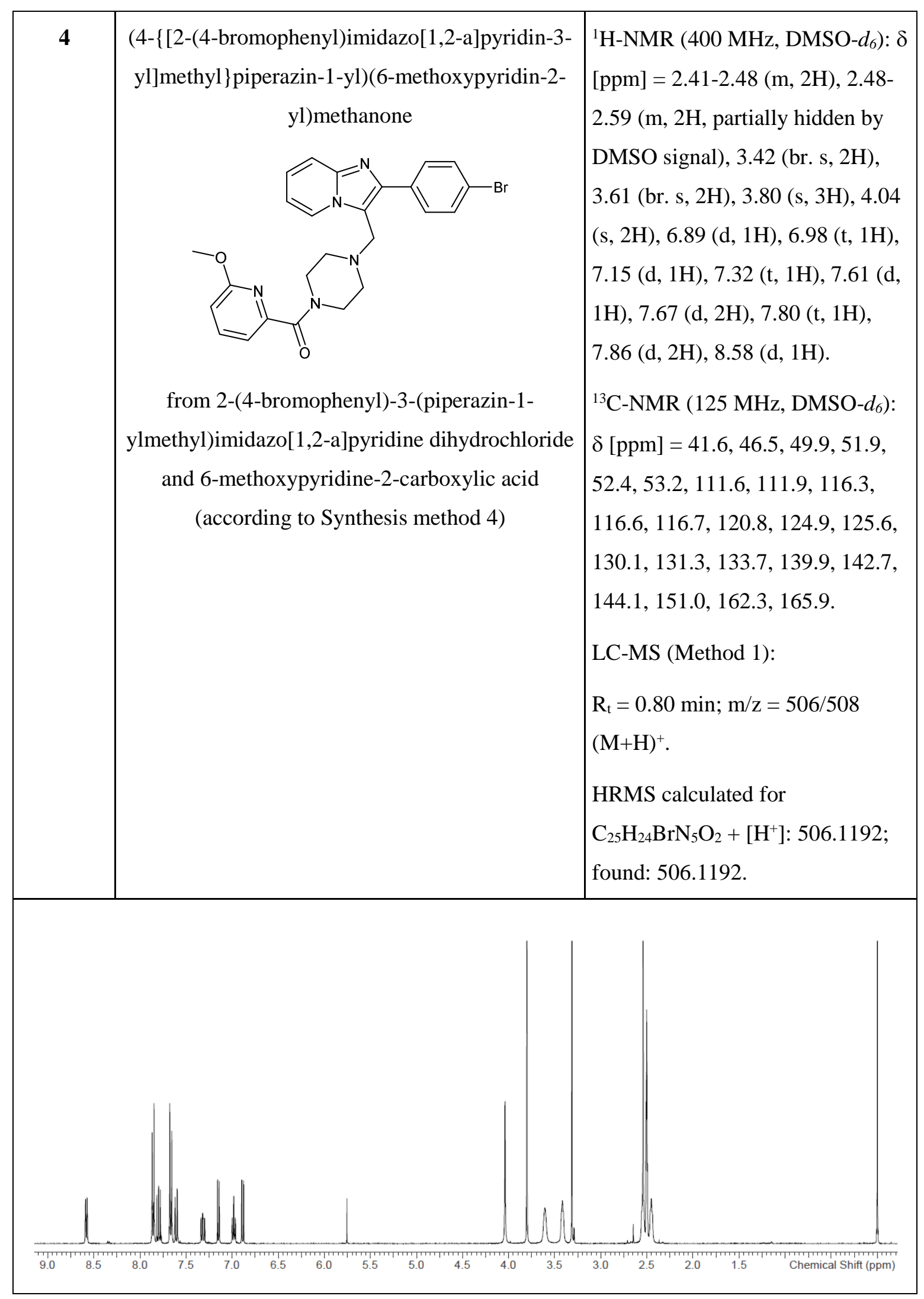




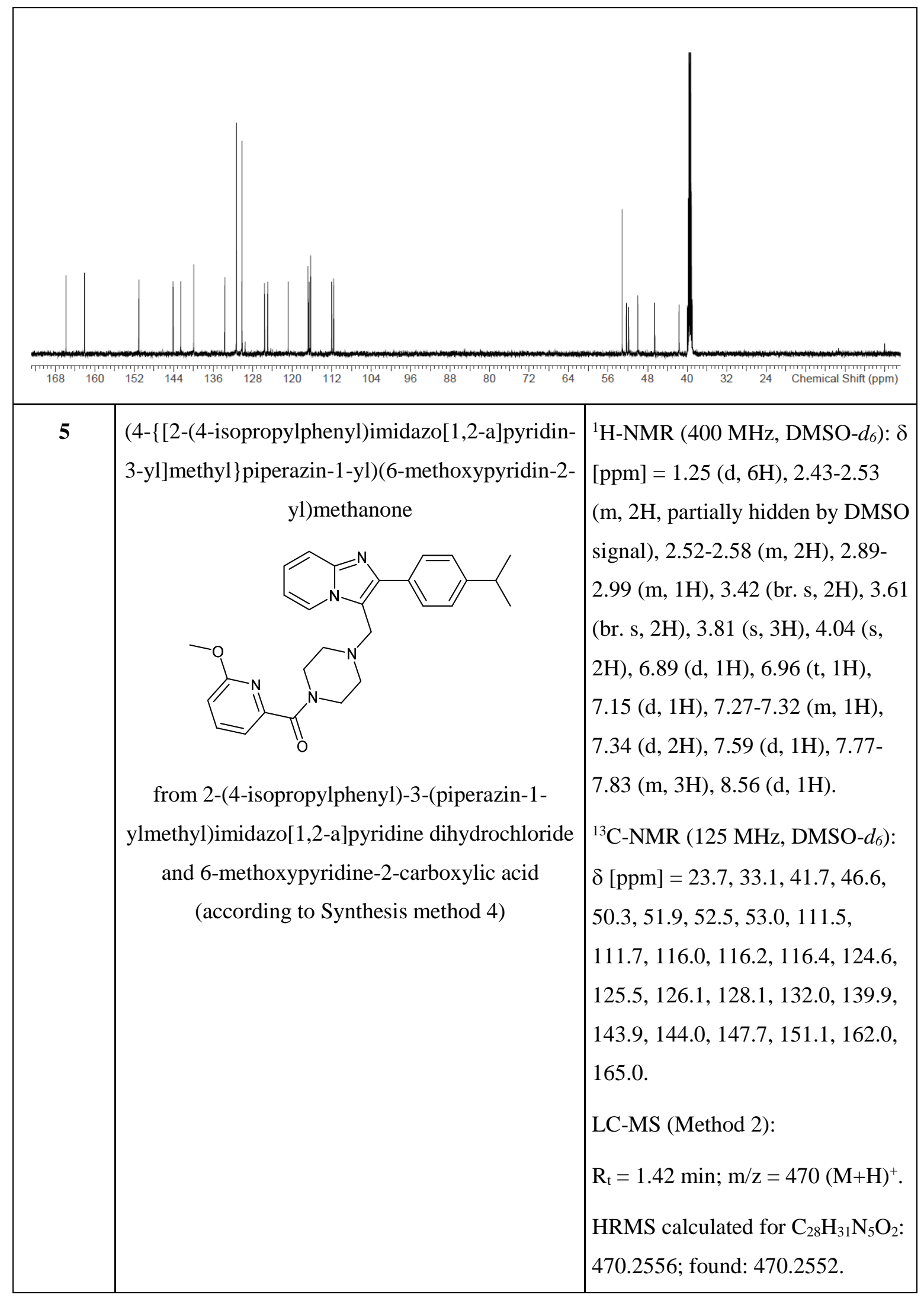




$$
\text { ست }
$$




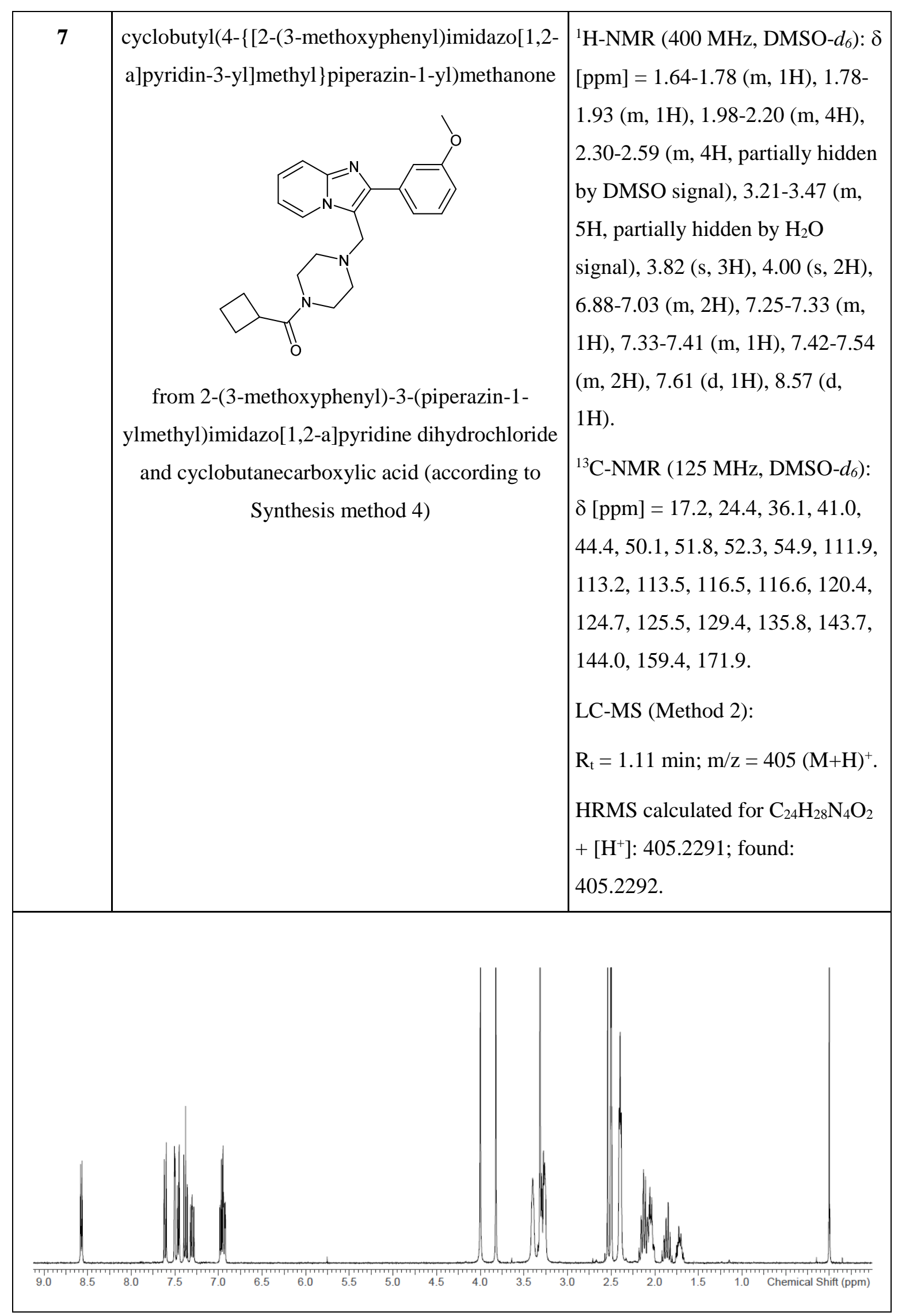




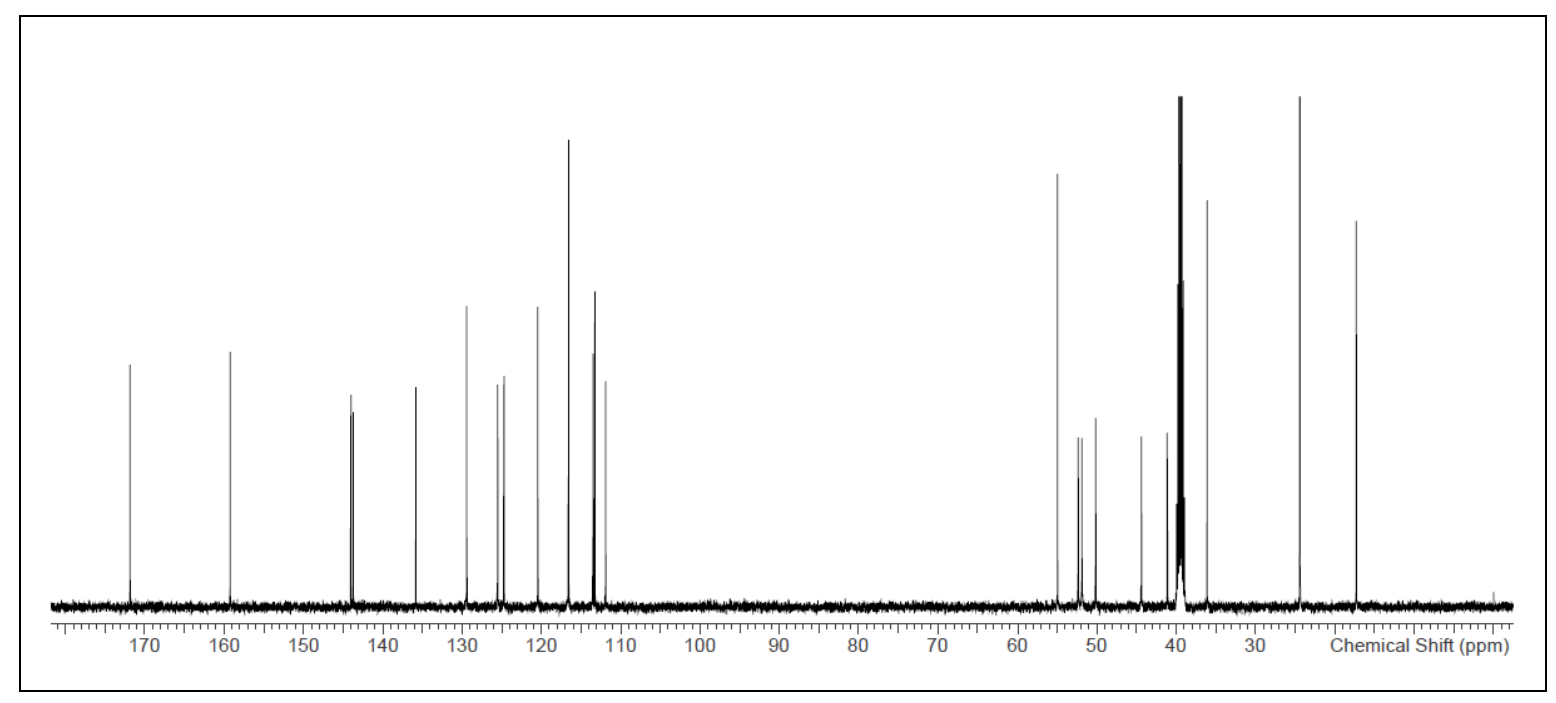


Supplementary Data

Supplementary Table 1 | Effects of BAY1000493 on a range of ion channels.

\begin{tabular}{cc}
\hline \multicolumn{2}{c}{$\begin{array}{c}\text { Voltage sensitive dye measurements } \\
\text { (FLIPR membrane potential assay) }{ }^{\mathbf{1}}\end{array}$} \\
\hline TRESK & $>30 \mu \mathrm{M}$ \\
TWIK-2 & $>30 \mu \mathrm{M}$ \\
EAG1 & $>30 \mu \mathrm{M}$ \\
$\mathrm{K}_{\mathrm{ir}} 3.1 / 3.4$ & $>30 \mu \mathrm{M}$ \\
$\mathrm{Kv} 7.1$ & $>10 \mu \mathrm{M}$ \\
$\mathrm{K}_{\mathrm{ca}} 3.1$ & $>30 \mu \mathrm{M}$ \\
\hline
\end{tabular}

Calcium sensitive dye measurements

(FLIPR Tetra System) ${ }^{2}$

\begin{tabular}{cc}
\hline $\mathrm{Ca}_{\mathrm{v}} 3.2$ & $>30 \mu \mathrm{M}$ \\
TRPC5 & $>30 \mu \mathrm{M}$ \\
TRPC6 & $>30 \mu \mathrm{M}$ \\
TRPA1 & $>30 \mu \mathrm{M}$ \\
TRPV4 & $>30 \mu \mathrm{M}$ \\
\hline Extended Cardiac Safety Profiler Panel (Eurofins) ${ }^{3}$ \\
\hline hERG & $2.4 \mu \mathrm{M}$ \\
$\mathrm{Na}_{\mathrm{v}} 1.5$ & $>30 \mu \mathrm{M}$ \\
$\mathrm{Ca}_{\mathrm{v}} 1.2$ & $>30 \mu \mathrm{M}$ \\
$\mathrm{K}_{\mathrm{v}} 1.1$ & $29 \mu \mathrm{M}$ \\
$\mathrm{K}_{\mathrm{v}} 1.3$ & $17 \mu \mathrm{M}$ \\
$\mathrm{K}_{\mathrm{v}} 1.5$ & $19 \mu \mathrm{M}$ \\
$\mathrm{K}_{\mathrm{v}} 4.3$ & $>10 \mu \mathrm{M}$ \\
$\mathrm{KCNQ} 1$ & $>30 \mu \mathrm{M}$ \\
$\mathrm{HCN} 4$ & $>30 \mu \mathrm{M}$
\end{tabular}

1.As described here described here: https://www.moleculardevices.com/sites/default/files/ en/assets/data- sheets/reagents/flipr-membrane-potential-assay-kits.pdf

${ }^{2}$ As described here: $\underline{\text { https://www.moleculardevices.com/sites/default/files/en/assets/data- }}$ sheets/reagents/calcium-assays-for-the-flipr-tetra-system.pdf

${ }^{3}$ As described here: https://www.eurofinsdiscoveryservices.com/cms/cms-content/services/in-vitroassays/ion-channels/cardiacprofiler/ 


\section{Supplementary discussion}

\section{Structure activity relationships for the inhibitors}

Shape complementarity seems to be the main component dictating the $\mathrm{IC}_{50}$ values for BAY1000493, BAY2341237 and the related compounds (Extended Data Table 2). Halogen substituents on the para position of the R1 phenyl ring are tolerated, whereas introduction of larger groups, such as a 4isopropanylphenyl group increases the $\mathrm{IC}_{50}$ value, for example compound $\mathbf{3}$ compared to analogue $\mathbf{5}$. Movement of the substituent on the phenyl group, from the para to the meta position is also disadvantageous, as it likely clashes with residues lining the TASK-1 fenestrations. Substitution of the cyclopentyl group to an 6-methoxypyridinyl group however is tolerated.

\section{Crystallographic features of the TASK-1 complexes with BAY1000493 and BAY2341237}

Both blockers bind in the vestibule below the selectivity filter and above the X-gate. They lie across the two-fold axis of the dimer and there is only space for one blocker molecule per dimer. The structure of the complex between TASK-1 and BAY1000493 showed a superposition of two copies of the blocker binding in the vestibule below the pore (Extended Data Fig. 6a,d). As BAY1000493 has a bromine atom, we were able to confirm this arrangement by collecting X-ray data at the bromine edge, then calculating an anomalous difference map. This map showed two symmetrical peaks in the vestibule, corresponding to the two orientations of BAY1000493 (Extended Data Fig. 6a). This observation can be explained by the fact that TASK-1 is a symmetrical homodimer and the blockers bind across the twofold axis of the dimer. Therefore, in each dimer only one molecule can bind in the vestibule. If the protein is entirely symmetrical then the surface of the two halves of the dimer will be very similar. If that is the case, then the probability of a dimer sitting one way around or the other in the crystal lattice are equal. If any local asymmetry at the compound binding site is not translated to the protein surface, then the protein can pack in either orientation, leading to a 50\% occupancy of the compound in either orientation (Extended Data Fig. 6g). This is what we observed for the BAY1000493 complex.

However, with the BAY2341237 complex the situation is different, as the inhibitor is clearly bound only in one orientation (Extended Data Fig. 6b,e). This observation can be explained if the blocker induces a slight asymmetry that propagates to the surface, leading to an overall asymmetrical dimer structure. In this case, when a dimer lands on the surface of the crystal, it will only be able to bind within the crystal lattice one way around, the induced changes at the surface would prevent it from binding the other way around (Extended Data Fig. 6h). In this case the orientation of the blocker within the vestibule will be $100 \%$ in one orientation within the crystal lattice.

The crystal contacts present in the TASK-1 structures are shown in Extended Data Fig. 2k and at the current resolution is it not possible to detect significant differences between the blocker bound complexes and the unbound protein. It is therefore unclear exactly how BAY2341237 induces asymmetry, although the presence of the tri-fluoromethyl group in the fenestration may be important. 
How asymmetry in the vestibule is propagated to the surface, ensuring asymmetrical binding within the crystals is also unclear and higher resolution structures will be needed to determine how asymmetry is induced.

\section{Disease associated variants in TASK channels, their location in the structure and predicted effects on function}

Mutations in TASK channels are associated with two diseases. Patients with mutations in TASK-1 suffers from autosomal dominant primary pulmonary hypertension type $4^{30}$ (PPH4, also known as pulmonary artery hypertension (PAH)). Inspection of the location of these variants in TASK-1 indicates that several of the mutations are associated with the pore (Gly97Arg and Gly203Asp), pore helix (Tyr192Cys) or extracellular loops at the ends of M3 and M4 (Glu182Lys and Val221Leu) (Extended Data Fig. 8). Mutation of Gly97 and Gly203 most likely influences ion conduction through the selectivity filter, as both these residues lie at the top of the selectivity filter. Tyr192 and Val221 are buried within the hydrophobic core of the protein, and in the case of Val221 is close to the lipid bilayer. Thus, changes in these residues may affect protein folding. However, one TASK-1 PPH4 causing mutation (Thr8Lys), does lie within the region of the X-gate latch (Fig. 3a, Extended Data Fig. 8c).

Patients with mutations in TASK-3 have Birk-Barel mental retardation dysmorphism syndrome, a severe developmental disorder with hypotonia and dysmorphism ${ }^{34}$. For example, the TASK-3 loss of function, maternally imprinted mutation, Gly236Arg, causes some cases of Birk-Barel mental retardation dysmorphism syndrome ${ }^{31}$. The mutation would insert two positively charged arginine sidechains into the vestibule, which we speculate could interfere with the movement of ions thorough the vestibule, and/or interfere with the X-gate (Extended Data Fig. 8h). Another Birk-Barel causing mutation, Ala237Asp ${ }^{32}$, involves an alanine residue, packed against the hydrophobic Ile132, so placing a larger, charged sidechain at this position, within the hydrophobic envelope of the membrane is likely to destabilize the protein fold. We found that the mutation Ala237Val in TASK-1 caused a substantial loss of expression, consistent with the suggestion that this mutation could destabilize the protein (Extended data Fig. 4a). However, further investigation of these mutations in TASK-1 and TASK-3 through functional, stability and structural studies will be essential to fully understand the effects of these mutations. 